\title{
Severity of Illness Measures of Rheumatoid Arthritis Using Thermal Infrared Imaging
}

\author{
by
}

Cynthia Adéa,

B.A.Sc. Electrical Engineering, University of Ottawa

\begin{abstract}
A thesis submitted to the Faculty of Graduate Studies and Research
In partial fulfilment of the requirements for the degree of

Master of Applied Science in Biomedical Engineering
\end{abstract}

\author{
Ottawa-Carleton Institute for Biomedical Engineering (OCIBME) \\ Department of Systems and Computer Engineering \\ Carleton University \\ Ottawa, Ontario, Canada, K1S 5B6
}

September 2009

(C) Copyright 2009, Cynthia Adéa 
Library and Archives

Canada

Published Heritage

Branch

395 Wellington Street

Ottawa ON K1A ON4

Canada
Bibliothèque et

Archives Canada

Direction du

Patrimoine de l'édition

395, rue Wellington

Ottawa ON K1A ON4

Canada
Your file Votre référence

ISBN: 978-0-494-60286-7

Our file Notre reférence

ISBN: 978-0-494-60286-7
NOTICE:

The author has granted a nonexclusive license allowing Library and Archives Canada to reproduce, publish, archive, preserve, conserve, communicate to the public by telecommunication or on the Internet, loan, distribute and sell theses worldwide, for commercial or noncommercial purposes, in microform, paper, electronic and/or any other formats.

The author retains copyright ownership and moral rights in this thesis. Neither the thesis nor substantial extracts from it may be printed or otherwise reproduced without the author's permission.
AVIS:

L'auteur a accordé une licence non exclusive permettant à la Bibliothèque et Archives Canada de reproduire, publier, archiver, sauvegarder, conserver, transmettre au public par télécommunication ou par l'internet, prêter, distribuer et vendre des thèses partout dans le monde, à des fins commerciales ou autres, sur support microforme, papier, électronique et/ou autres formats.

L'auteur conserve la propriété du droit d'auteur et des droits moraux qui protège cette thèse. $\mathrm{Ni}$ la thèse ni des extraits substantiels de celle-ci ne doivent être imprimés ou autrement reproduits sans son autorisation.
In compliance with the Canadian Privacy Act some supporting forms may have been removed from this thesis.

While these forms may be included in the document page count, their removal does not represent any loss of content from the thesis.
Conformément à la loi canadienne sur la protection de la vie privée, quelques formulaires secondaires ont été enlevés de cette thèse.

Bien que ces formulaires aient inclus dans la pagination, il n'y aura aucun contenu manquant.

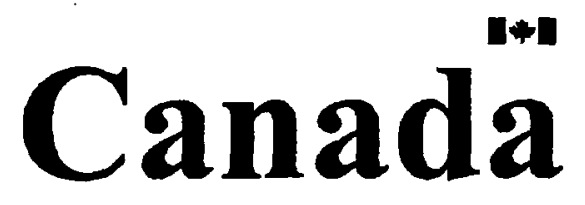




\section{ABSTRACT}

This research presents thermal infrared (TIR) imaging systems as a potential effective tool for physicians to diagnose and treat Rheumatoid Arthritis (RA). TIR images were taken of 18 control subjects and 13 patients diagnosed with RA by a rheumatologist. Mathematical measurements were extracted from the joints in the hand, wrist and knee images and analysed with statistical tests to show differences between the control and patient groups. The $2^{\text {nd }}$ metacarpophalangeal, the $3^{\text {rd }}$ metacarpophalangeal and the knee were the joints that showed the greatest statistical difference between control subjects and patients. The best measurements extracted from the TIR images were variance, maximum-minimum temperature difference and mode/maximum temperature ratio. RA patients were classified successfully into three RA severity groups using a ratio measurements calculated from the raw data of the images. Finally, the effect of age on the TIR measurements of the control and the patient groups was found to be weak. 


\section{ACKNOWLEDGEMENTS}

First and far most, I would like to thank God for being my ultimate rock and my teacher throughout my engineering masters. This work was done heartily for Him (Colossians 3:23) the One I serve.

I would like to thank my supervisor Dr. Monique Frize for her constant support and encouragement over the course of my engineering academic career, and for her enthusiasm in introducing me to the field of biomedical engineering. It is that exposure to medicine that has helped shape my career path and future endeavors in medicine. I would like to express my appreciation to Dr. Monique Frize for her personable approach as a supervisor and for her willingness to invest in young students with potential early on in their academic walk.

I would like to express my gratitude to current and past members of the Medical Information-technologies Research Group for their guidance and advice. Their help has been pivotal throughout the completion of this thesis.

I would like to thank my parents for the love and support they have given me throughout this process. I would like to thank them for always encouraging me to aim high and work hard in all I pursue.

Finally, I would like to thank my husband Donald who has been there since the beginning of my engineering career and who has been the Godly support that has motivated me to complete this work and pursue my dreams. I would like to express my gratitude for the way he has lead me through the completion of this thesis (Ephesians 5: 22-33). 


\section{TABLE OF CONTENTS}

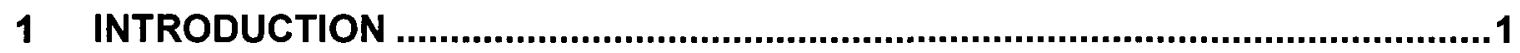

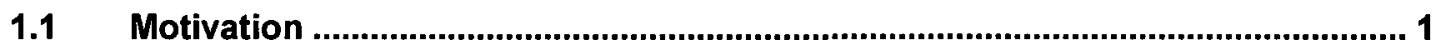

1.2 Definition of Rheumatoid Arthritis ............................................................... 3

1.3 Epidemiology of Rheumatoid Arthritis ......................................................... 4

1.4 Pathophysiology of Rheumatoid Arthritis .......................................................... 5

1.5 Symptoms of Rheumatoid Arthritis ................................................................... 7

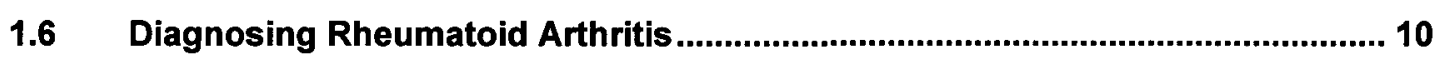

1.7 Treatment and Therapy of Rheumatoid Arthritis ............................................ 15

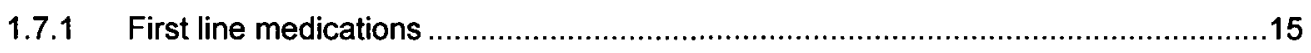

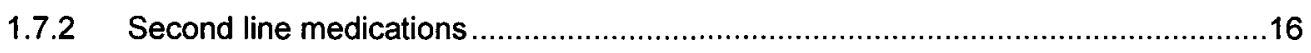

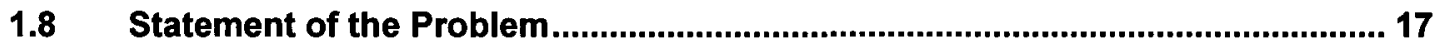

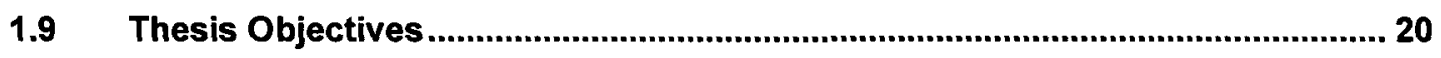

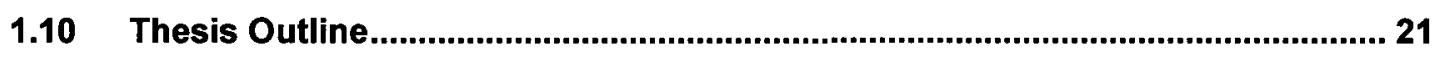

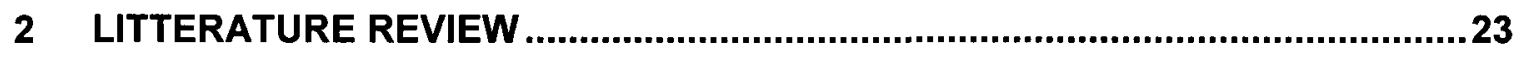

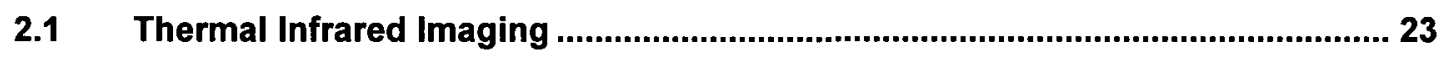

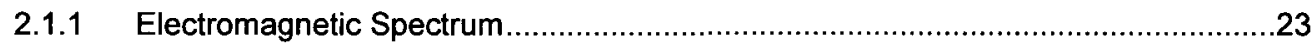

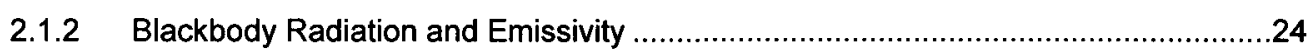

2.1.3 Planck's Law and Wien's Displacement Law ....................................................25

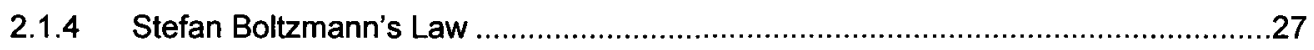

2.2 Past Research in Infrared Thermography .................................................. 29

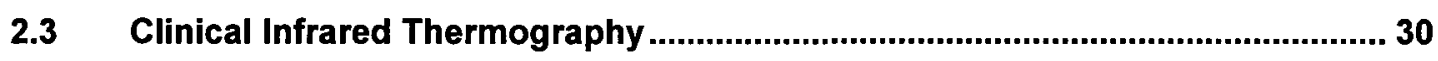

2.3.1 General Medical Applications of TIR Technology …............................................32

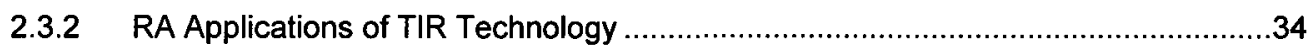




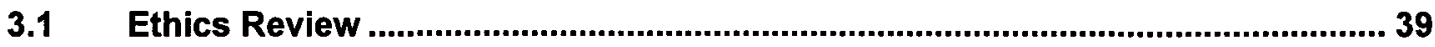

3.2 Recruitment of Participants ............................................................................... 40

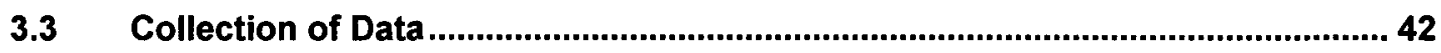

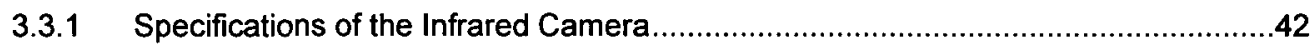

3.3.2 Infrared Camera and Recording Settings............................................................

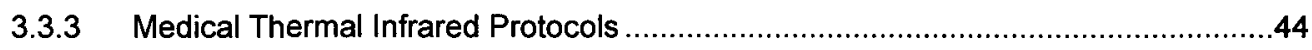

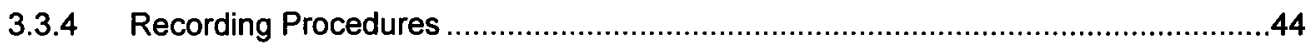

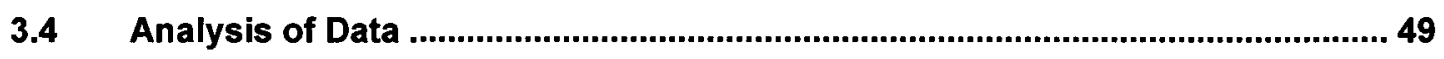

3.4.1 Converting the Thermal IR Images into Data for analysis .....................................50

3.4.2 First Objective - Discriminate Between the Control Group and the Patient Group...55

3.4.3 Second Objective - Identify the Joints that Best Determine if a Participant has or

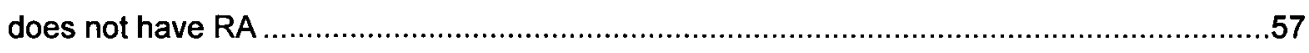

3.4.4 Third Objective - Identify the Matrix that Best Assesses the Presence or Absence of RA $\quad 59$

3.4.5 Fourth Objective - Classify RA Patients into Three Severity Groups ......................61

3.4.6 Fifth Objective - Observe the Effect of Age on Thermal IR Measurements..............61

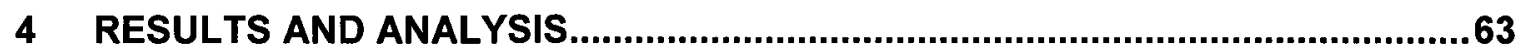

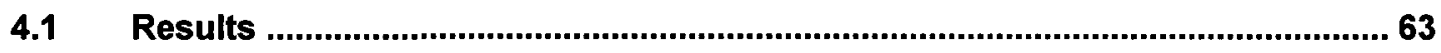

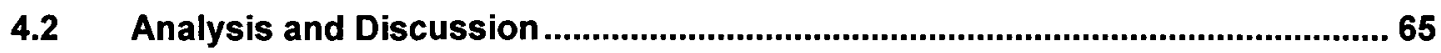

4.2.1 First Objective - Discriminate Between the Control Group and the Patient Group ...65

4.2.2 Second Objective - Identify the Best Joints that Best Determines whether a

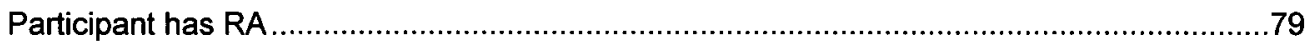

4.2.3 Third Objective - Identify the Matrix that Best Assesses the Presence or Absence of RA $\quad 81$

4.2.4 Fourth Objective - Classify RA Patients into Three Severity Groups .....................85

4.2.5 Fifth Objective - Observe the Effect of Age on Thermal IR Matrices......................89 
5 CONCLUSION.

$5.1 \quad$ Contributions to Knowledge ............................................................................. 95

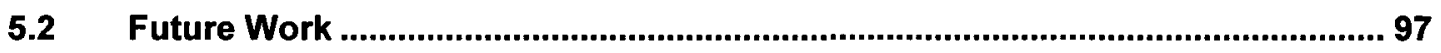

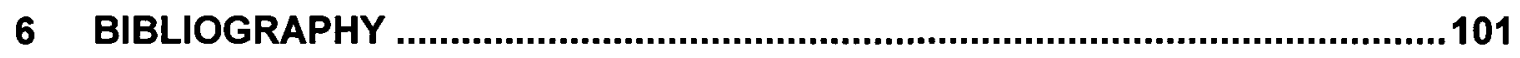

7 APPENDIX A

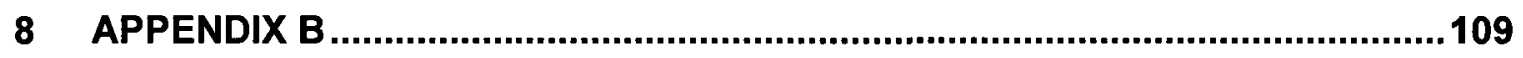

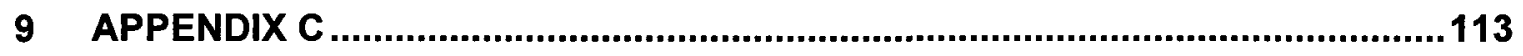

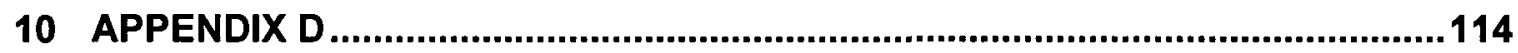

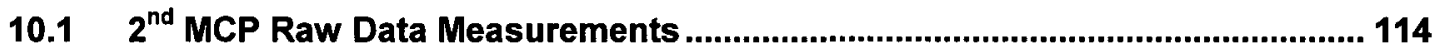

$10.23^{\text {rd }}$ MCP Raw Data Measurements .................................................................... 116

$10.3 \quad 2^{\text {nd }}$ PIP Raw Data Measurements ..................................................................... 118

$10.43^{\text {rd }}$ PIP Raw Data Measurements....................................................................... 120

10.5 Wrist Raw Data Measurements ......................................................................... 122

10.6 Knee Raw Data Measurements ............................................................................. 124

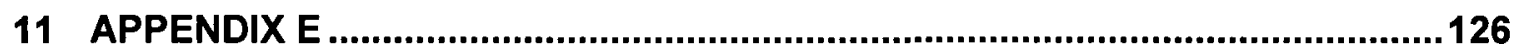

11.1 Histogram of $2^{\text {nd }}$ MCP's Most Statistically Significant Matrices ....................... 126

11.2 Histogram of $3^{\text {rd }}$ MCP's Most Statistically Significant Matrices ....................... 128

11.3 Histogram of the Knee's Most Statistically Significant Datasets..................... 129

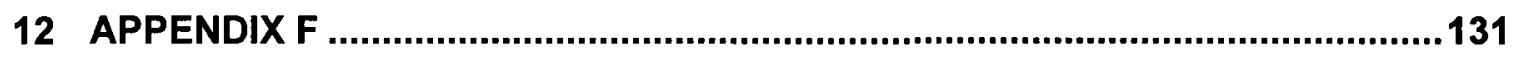




\section{LIST OF FIGURES}

Figure 1-1: Normal Synovial Joint........................................ 4

Figure 1-2: Pathological Features of Rheumatoid Arthritis ..................... 6

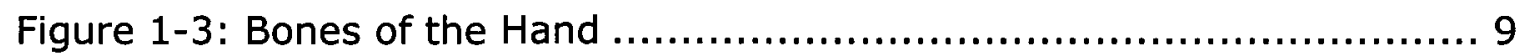

Figure 1-4: Bones of the Foot (A) Anterior View (B) Lateral View.............. 9

Figure 1-5: The Joint Distribution of Rheumatoid Arthritis and Osteoarthritis

Figure 1-6: Radiographic Features of an RA Joint.............................13

Figure 2-1: Electromagnetic Spectrum (FLIR Systems, 2004).................24

Figure 2-2: Emissive Power of a Blackbody at Different Temperatures and Wavelenghts According to Planck's Radiation Law (FLIR Systems, 2004)

Figure 2-3: Planckian Curves Plotted on Semilog Scale (FLIR Systems, 2004)

Figure 3-1: A40M Infrared Camera from FLIR Systems FLIR Systems, 2004)

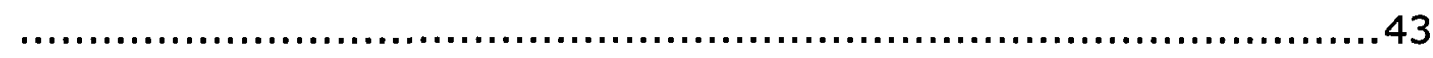

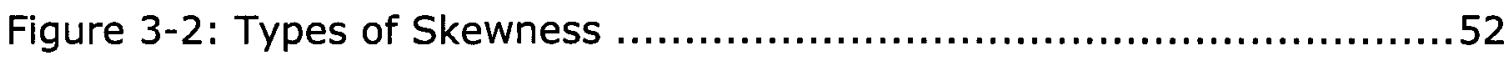

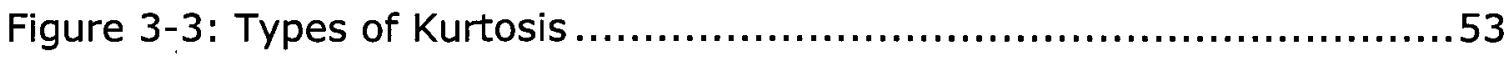

Figure 4-1: Histogram of the Mode/Max Matrix for the 2nd MCP .............71

Figure 4-2: Cluster Chart of Mode/Max Matrix of the 2nd MCP of the Control

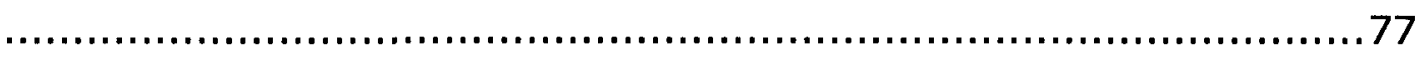

Figure 4-3: Histogram of the Mode/Max Matrix of the 3rd MCP ................80

Figure 4-4: Histogram of the Variance Matrix of the Knee......................82 
Figure 4-5: Average of the Patient's 2nd MCP Mode/Max Matrix Elements...88 Figure 4-6: Average of the Control's 2nd MCP Mode/Max Matrix Elements by Age Group

Figure 5-1: Block Diagram of Christophe Herry's Thesis Research (2008) ...99 Figure 11-1: Histogram of the Mode/Max Matrix for the 2nd MCP 126

Figure 11-2: Histogram of the Mode/Min Matrix for the 2nd MCP ........... 127

Figure 11-3: Histogram of the Skewness Matrix for the 2nd MCP 127

Figure 11-4: Histogram of the Variance Matrix for the 3rd MCP ............. 128

Figure 11-5: Histogram of the Mode/Max Matrix for the 3rd MCP ........... 128

Figure 11-6: Histogram of the Max-Min Matrix for the 3rd MCP..............129

Figure 11-7: Histogram of the Variance Matrix of the Knee .................. 129

Figure 11-8: Histogram of the Mode/Min Matrix of the Knee................. 130

Figure 11-9: Histogram of the Max-Min Matrix of the Knee .................. 130 


\section{LIST OF TABLES}

Table 1-1: American College of Rheumatology's Criteria for the Classification of Rheumatoid Arthritis (Arnett 1998, 315-324) ..........................14

Table 3-1: Grey Level Thermal Infrared Views of the Elbow ..................46

Table 3-2: Grey Level Thermal Infrared Views of the Wrist and Hand ........46

Table 3-3: Grey Level Thermal Infrared Views of the Knee....................47

Table 3-4: Gray scale Level Thermal Infrared Views of the Ankle and Foot..48 Table 3-5: Regions of Interests Manually Selected for the $2^{\text {nd }}$ and $3^{\text {rd }}$ MCPS, the $2^{\text {nd }}$ and $3^{\text {rd }}$ PIPs, the Wrist and the Knee.............................51

Table 3-6: The Binary Image Output of MATLAB's roicolor Function which Highlights the Pixels that are in the Mode to Maximum Temperature

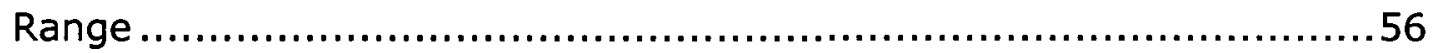

Table 3-7: Anatomical Views of the Hand and Knee Used for Analysis ........59

Table 4-1: Average Values for Each Matrix of Every Joint for the Control and Patient Group...............................................................64

Table 4-2: MATLAB Lilliefors Test Results for Randomly Selected Matrices ..67 Table 4-3: MATLAB's Ranksum Test Results Comparing the Matrix Distributions of the Control and the Patient Group 69

Table 4-4: Grey Images and their Corresponding Binary Images of Control Subjects................................................................... 72

Table 4-5: Grey Images and their Corresponding Binary Images of Patients73 
Table 4-6: Percentage of Pixels Within the Range Mode to Maximum Temperature Representing the Area of the Joint that is Covered with Warmer Pixels and/or Hot Regions...................................... 75

Table 4-7: Physician's Assessment of Each Patient and........................ 85

Table 4-8: Ranking of the Patient's 2nd MCP Mode/Max Matrix ................86

Table 4-9: Spearman Correlation Coefficient Calculation Between the Physician Ranking and the Matrix Ranking ...............................8 87

Table 4-10: Control Subjects with their Corresponding .......................90

Table 4-11: Ranking of the Control's 2nd MCP Mode/Max Matrix..............91

Table 4-12: Spearman Correlation Coefficient Calculation .....................92

Table 10-1: Raw Data Measurements Extracted from the Control Group's 2nd MCP Joint 114

Table 10-2: Raw Data Measurements Extracted from the Patient Group's 2nd MCP Joint 115

Table 10-3: Raw Data Measurements Extracted from the Control Group's 3rd MCP Joint 116

Table 10-4: Raw Data Measurements Extracted from the Patient Group's 3rd MCP Joint 117

Table 10-5: Raw Data Measurements Extracted from the Control Group's 2nd PIP Joint 118

Table 10-6: Raw Data Measurements Extracted from the Patient Group's 2nd PIP Joint 
Table 10-7: Raw Data Measurements Extracted from the Control Group's 3rd PIP Joint. 120

Table 10-8: Raw Data Measurements Extracted from the Patient Group's 3rd PIP Joint. 121

Table 10-9: Raw Data Measurements Extracted from the Control Group's Wrist Joint 122

Table 10-10: Raw Data Measurements Extracted from the Patient Group's Wrist Joint 123

Table 10-11: Raw Data Measurements Extracted from the Control Group's Knee Joint 124

Table 10-12: Raw Data Measurements Extracted from the Patient Group's Knee Joint 125 


\section{LIST OF ACRONYMS}

\begin{tabular}{|c|c|}
\hline ATR & Automated Target Recognition \\
\hline DIP & Distal InterPhalangeal \\
\hline DMARDs & Disease-Modifying Anti-Rheumatic Drugs \\
\hline HDI & Heat Distribution Index \\
\hline HRUS & High Resolution Ultra Sound \\
\hline IL-1 & InterLeukin-1 \\
\hline IR & InfraRed \\
\hline MCP & MetaCarpoPhalangeal \\
\hline MIRG & Medical Information-technologies Research Group \\
\hline MRI & Magnetic Resonance Imaging \\
\hline MTP & MetaTarsal Phalangeal \\
\hline NSAIDs & NonSteroidal Anti-Inflammatory Drugs \\
\hline OHREB & Ottawa Hospital Research Ethics Board \\
\hline PIP & Proximal InterPhalangeal \\
\hline RA & Rheumatoid Arthritis \\
\hline STD & Standard Deviation \\
\hline TI & Thermal Index \\
\hline TIR & Thermal InfraRed \\
\hline WHO & World Health Organization \\
\hline X-Ray & Radiography \\
\hline
\end{tabular}




\section{CHAPTER 1}

\section{INTRODUCTION}

This chapter begins by introducing the motivation of this thesis and the statement of the problem. It continues with a detailed background of rheumatoid arthritis - definition, epidemiology, pathophysiology, symptoms, diagnosis and treatment. Such detail on rheumatoid arthritis disease is necessary to better understand the specific requirements of thermal infrared imaging analysis in future chapters. This chapter ends with the thesis objectives and outline.

\subsection{Motivation}

In 2000, the World Health Organization reported Rheumatoid Arthritis (RA) as the $31^{\text {st }}$ worst leading cause of "Years Lived with Disability" at a global level (Symmons 2006). RA is a disabling disease, more so than lower limb osteoarthritis. The disability can begin early and rise in a linear fashion (Hochberg 1992, 498-502). Within ten years of onset, $50 \%$ of patients in developing countries are unable to hold down full-time jobs (Brooks 1997). RA also reduces life expectancy by 5 to 10 years (Guedes 1999, 492-298). 300000 Canadians, 2.1 million Americans and approximately 260000 French citizens are 
currently diagnosed with RA. There is therefore a huge need to find a tool that can better assist doctors in diagnosing and treating people inflicted with RA.

The motivation of this research is to use InfraRed (IR) thermal imaging to provide quantitative assessment of joints to assist physicians in diagnosing RA very early on and in monitoring treatment via the deterioration of the joints. This would be a major innovation in Canada and worldwide. IR imaging technologies are safe non-ioninzing tools that are inexpensive, non-invasive and capable of examining multiple joints per image in a reasonable scanning time period. Replacing the current RA modalities - Magnetic Resonance Imaging (MRI) and High Resolution Ultra Sound (HRUS) - by IR technology would have a major impact on the Health Care System. For instance, thermal IR tests would release expensive imaging tests, such as MRI, for other medical applications thus reducing the wait times for these procedures.

Furthermore, IR imaging would improve the prognosis of RA patients by detecting the disease early and allowing the doctor to employ an aggressive treatment at an early stage. A better prognosis means an RA patient with controlled pain and a slower progression of the deformation of the joint. Therefore employing IR technologies would greatly improve the quality of life of patients and allow them to remain active for a longer period of time. They could remain employed and be contributing members of society for a longer period of time.

Moreover, IR imaging techniques can help physicians in managing and treating a patient's pain and disease progression by quantifying the inflammation 
of each joint during the medicated period. This would diminish the length of the "trial and error period" for finding a first or second line medication match for the RA patient by making the process more efficient by quantifying the trial results for physicians. In addition, IR technologies could identify when a patient is in remission or in a flaring period to help the doctor adjust doses when needed. The goal of the physician is to achieve the lowest possible level of disease activity. IR image measurements could therefore show physicians when the treatments are effective.

Overall, IR thermal imaging is a technology that should be explored as an application to assist physicians in diagnosing and managing RA because of its great potential impact on the Health Care System and on society as a whole.

\subsection{Definition of Rheumatoid Arthritis}

$\mathrm{RA}$ is an autoimmune disease triggered by the exposure of a susceptible host to a microbial antigen. The body's immune response to this antigen causes a systemic inflammatory response which leads to polyarthritis manifested mainly in the synovial membrane of joints and chronic inflammation of the tissue around the joints. Other organs of the body such as the lungs or eyes may also be affected. This inflammatory reaction releases inflammatory mediators that cause damage to the synovial membrane, the cartilage and the bone. The end result is cartilage loss, joint damage and functional impairment of the joints. Damage to the joints can occur early in the disease and be progressively leading to the deformation of the joint (Lipsky 2008). It will therefore be important that the IR 
thermal technology be able to detect not only bone changes, but soft tissue changes as well - especially damage to the synovial membrane. This will allow the thermal infrared camera user to differentiate between degenerative osteoarthritis (bone and cartilage damage) and RA (inflammation of the synovial membrane). The figure below illustrates a normal synovial joint.

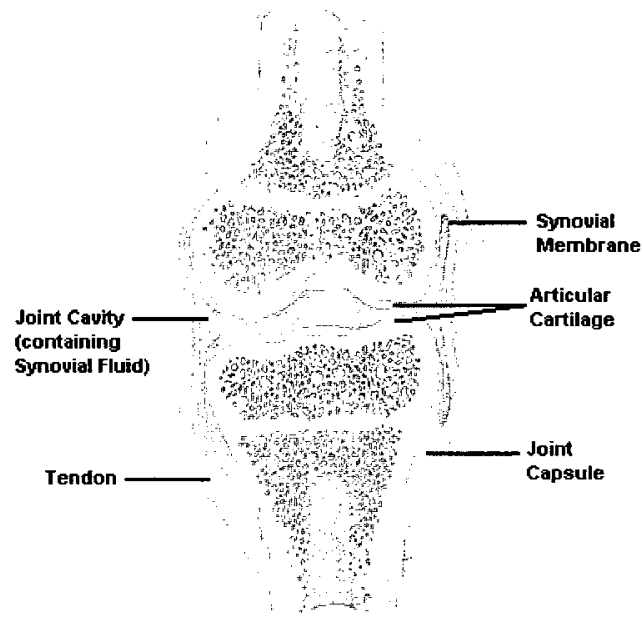

Figure 1-1: Normal Synovial Joint

\subsection{Epidemiology of Rheumatoid Arthritis}

The prevalence of the disease worldwide ranges from 0.3 to $2.1 \%$. It affects women three times more than men and all races are affected equally. The prevalence increases with age while sexe differences decrease with age. RA can begin at any age, but $80 \%$ of patients develop RA between the ages of 35 and 50 . The incidence of RA is more than six times greater for women between 60 to 64 years of age compared to women between 18-29 years of age. Recent studies have shown that the incidence and the severity of RA may be diminishing. This may be a reflection of more aggressive therapeutic interventions. Furthermore, studies have shown a genetic predisposition to RA 
since it is common to observe first degree relatives of patients with RA to also be diagnosed with RA (Lipsky 2008). This epidemiologic information helped determine the inclusion and exclusion criteria of our participant database and supported the engineering analysis and discussions of the results. These criteria will be specified in chapter 3 of methodology and the analysis and discussions are presented in chapter 5 .

\subsection{Pathophysiology of Rheumatoid Arthritis}

The exact cause of RA is unknown. However, many studies have explored how the body's immune system functions and interacts to cause autoimmune diseases such as RA. The immune system's role is to seek and destroy antigens that invade the body by producing antibodies that target the invader. People with autoimmune diseases produce antibodies that mistakenly attack specific tissues of their own body.

RA begins with the exposure of an immunocompromised host to an unknown antigen. The antigen is presented to the body via macrophages resulting in the proliferation of T-cells - a type of immune cell lymphocyte. Cytokines are released by the T-cells which activate the proliferation of B-cells another type of immune cell lymphocyte. The B-cells produce the autoantibodies that attack the unknown antigen but that also attack the body's synovial membranes. An inflammatory reaction ensues and the cartilages along with the bone are damaged. The resulting joint inflammation causes swelling, stiffness, redness and pain in the joints (El-Gabalawy 2001). 
Alongside the production of the auto-antibodies by the B-cells, the macrophages release a chemical mediator - interleukin-1 (IL-1). IL-1 causes the proliferation of synovial cells, the activation of cartilage remodelling cells chondrocytes - and the proliferation of blood vessels in the synovial membrane. The synovial cell proliferation leads to the disorderly growth of the synovial membrane which results in a pannus that invades the neighbouring cartilage ultimately leading to cartilage and bone destruction. The activated chondrocytes excessively release cartilage digestive enzymes which lead to the degradation of cartilage and eventually of bone. Finally, the proliferation of blood vessels in the synovial membrane increases the vascular permeability of the joint membranes resulting in synovial oedema - the excessive accumulation of synovial fluid in the synovium of the joint. This increased vascularisation also leads to the accumulation of B- and T-cells in the synovium which further contributes to the joint inflammation and damage (Haraoui 2001). The figure below exhibits the joint changes in RA.

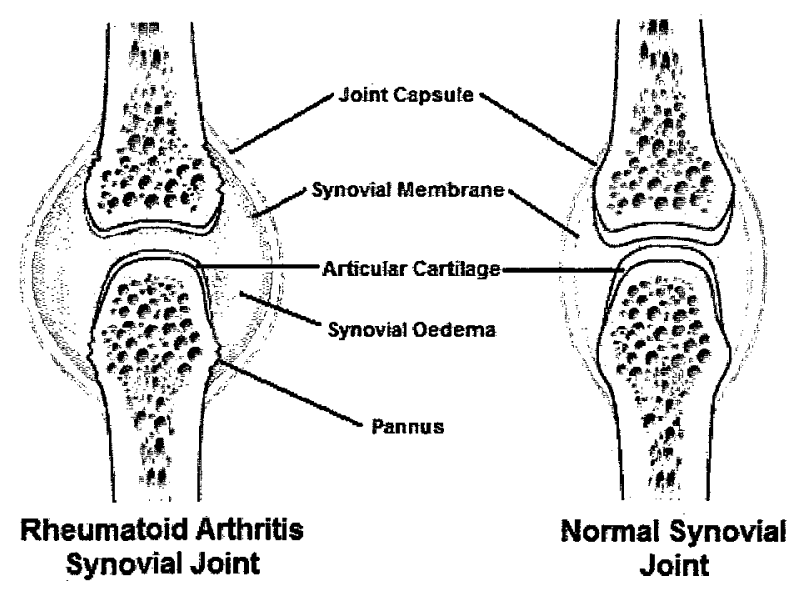

Figure 1-2: Pathological Features of Rheumatoid Arthritis 
The above figure, illustrates the main pathological feature of RA synovitis - the inflammation, proliferation and invasion of the synovial membrane. In the normal joint, the synovial lining layer is one to two cells thick with blood vessels found in the deeper layers of the loose connective tissue of the joint. In $\mathrm{RA}$, the lining layer is six to ten cells thick with an increased number of blood vessels. Another pathological observation in the figure above is the cartilage and bone degradation. The erosion is primarily found in the pannus region where the fibrous capsule of the joint that is lined with the synovial membrane meets with the cartilage and bone.

The specific pathophysiology of the disease is important to understand when interpreting the IR thermal images. The next chapter presents passed research that shows that IR thermal images reveal evidence of chemical changes subcutaneously, such as the release of inflammatory chemical mediators in RA (Ring 1970, 337 and Collins 1970, 386). Synovial oedema can also change the intra-articular temperature and would therefore show a change in the skin surface temperature and be evidence of disease activity (Bacon 1976, 51-65). Furthermore, IR imaging modalities can detect cellular proliferation and increase in vascular flow, which are the phathophysiological evidences of an RA pannus (Nielsson 1980, 516-528).

\subsection{Symptoms of Rheumatoid Arthritis}

The degree of the symptoms of RA depends on the degree of the tissue inflammation. Patients with RA are identified as having "active" disease when 
the body tissues are inflamed. "Remission" occurs when the symptoms of the disease are absent and the inflammation subsides. Remission can arise with treatment or without and can last weeks to years. The symptoms of RA can return and inflammation can become active at any time. This is called a period of "flare". The course of RA can greatly vary from patient to patient with periods of remissions and flares.

When RA is active, the systemic symptoms may include lack of appetite, fatigue, low-grade fever, muscle and joint aches and stiffness. Muscle and joint stiffness appear more commonly in the morning for more than an hour and after periods of inactivity. Joint inflammation is common during flare periods, where joints are warm, red, painful, swollen and tender. It is important to note that the progression of the disease and the degree of damage to the joints does not necessarily correlate with the degree of pain, stiffness or swelling present in the joints.

RA affects multiple joints in a symmetrical bilateral pattern (affecting both sides of the body). The small joints of the hands, the wrists and the feet are usually affected. These joints more specifically include the proximal interphalangeal (PIP) joints, the metacarpophalangeal (MCP) joints, the wrist and the metatarsal phalangeal (MTP) joints. The distal interphalangeal (DIP) joints are not affected by RA. The figures below clearly display the location of these joints. 


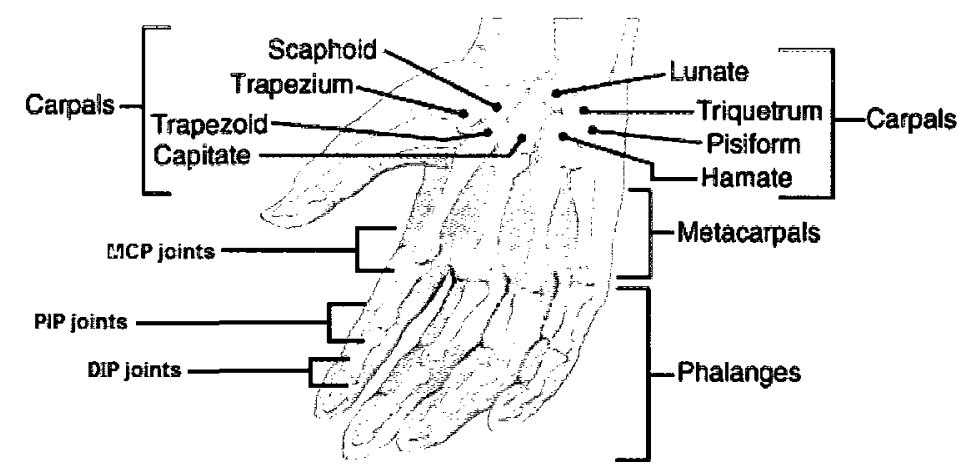

Figure 1-3: Bones of the Hand

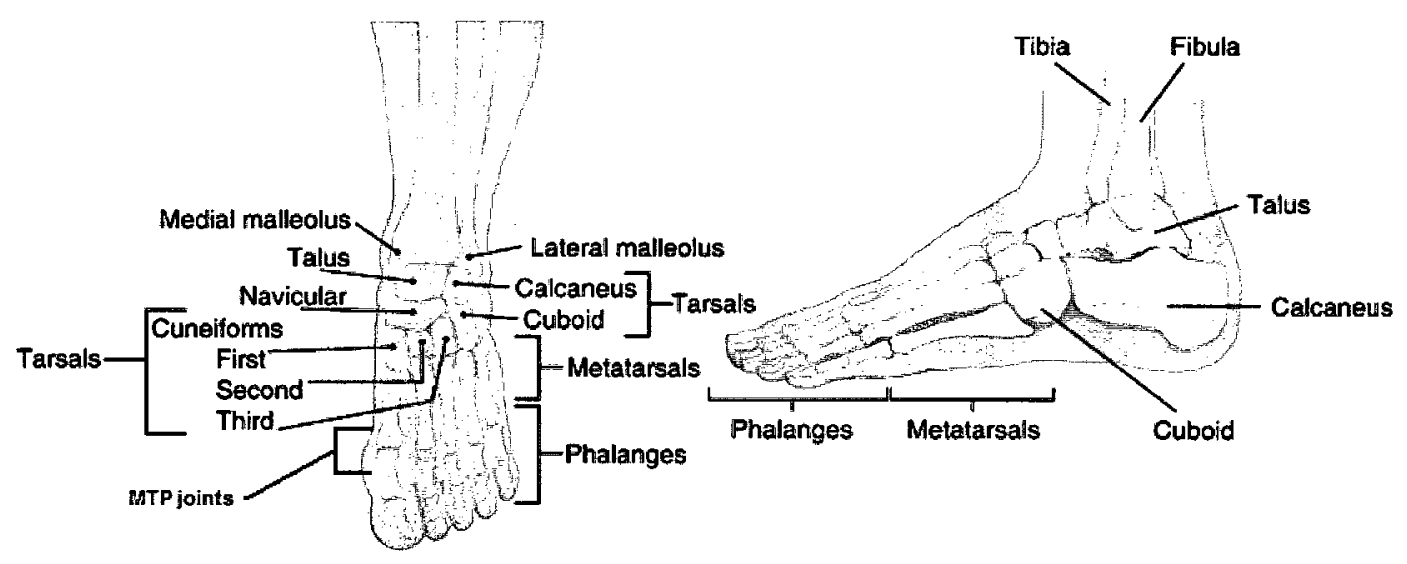

Figure 1-4: Bones of the Foot (A) Anterior View (B) Lateral View

Chronic inflammation can result in damage to the body tissues, cartilage and bone. Consequently, there is a loss of cartilage; erosion and weakness of bones and muscles; and ultimately joint deformity, destruction and loss of function.

RA is systemic and therefore can involve the inflammation of other organs and areas of the body, not only joints. The glands of the eyes and mouth can be inflamed causing dryness to these areas. Inflammation of the pleura of the lung - pleuritis - can cause chest pain and coughing. Rheumatoid nodules (firm lumps of inflammation) can also be found in the lung tissue itself. Asymptomatic 
rheumatoid nodules can be present around the elbows and fingers. Inflammation of the heart's pericardium - pericarditis - can cause positional chest pain. An inflamed and enlarged spleen can cause anemia as well as a decrease in white blood cells. Anemia leads to fatigue, dizziness or lightheadedness, palpitations, difficulty breathing (dyspnea), headache and pallor. A decrease in white blood cells makes the patient more susceptible to infections. Blood vessel inflammation - vasculitis - is a rare and serious complication of chronic rheumatoid disease which can lead to tissue death. Tissue death can be identified as tiny visible black areas around the nail beds or as leg ulcers (O'Dell 2008, chapter 15).

Thermal IR images can detect RA symptoms such as swelling, inflammation, heat and redness. It has also been proven that pain can be detected by this non-invasive technology (Herry 2004, 19). This thesis will go on to prove that these symptoms are quantifiable via IR thermography measurements. The systemic RA changes above, such as nodules and vascular changes, may be useful in understanding the temperature distribution spikes and anomalies around the observed joints and limbs.

\subsection{Diagnosing Rheumatoid Arthritis}

The diagnosis of RA is done by a doctor by initially reviewing the patient's history and performing a physical exam. The doctor carefully takes note of the patient's symptoms and performs the classical examination technique of palpation to examine the joints for inflammation and deformity, the skin for 
nodules and other parts of the body for signs of inflammation - redness, enlargement, warmth and tenderness.

The doctor also identifies the distribution and the range of motion of the affected joints. The distribution of the inflamed joints is important in the diagnosis of RA. RA does not have a predilection for weight bearing joints. The small joints of the hands (the proximal interphalangeal (PIP) joints and the metacarpophalangeal (MCP) joints), the wrists, the metatarsal phalangeal (MTP) joints of the feet and the knees are often the target and are inflamed in a symmetrical pattern (meaning both sides of the body are affected). When only one joint unilaterally is inflamed, the diagnosis of RA is more difficult and requires other tests.

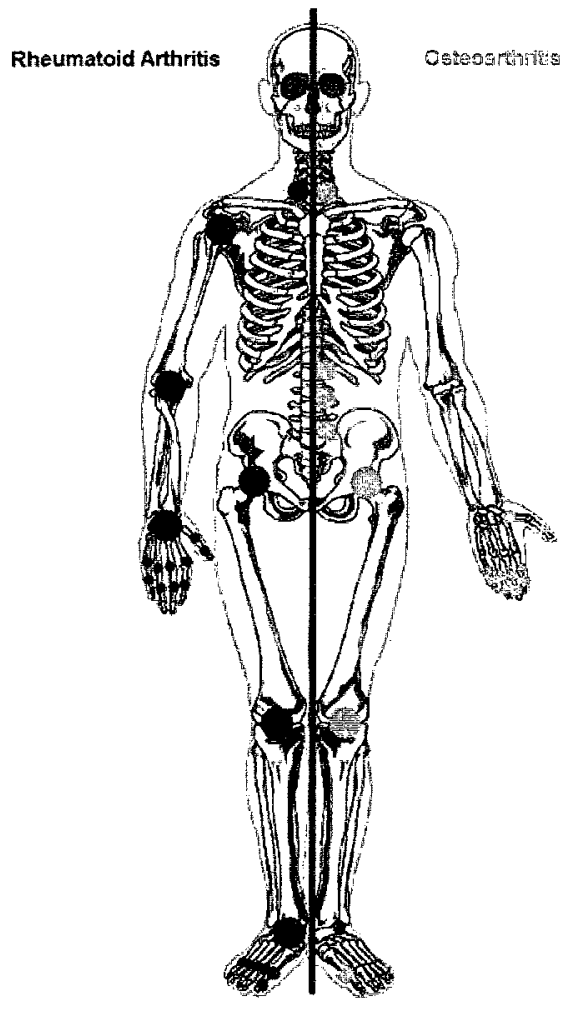

Figure 1-5: The Joint Distribution of Rheumatoid Arthritis and Osteoarthritis 
The physician may request blood tests to help confirm the diagnosis. These tests can show abnormal serum antibodies, including a serum antibody called the rheumatoid factor which is present in $80 \%$ of RA patients. Citrulline antibody and antinuclear antibody are also frequently found in patients with RA. Another blood test called the sedimentation rate is used to observe the speed at which the red blood cells sink to the bottom of a test tube. The sedimentation rate is an indicator of inflammation and increases during flare periods and decreases during remission periods. The C-reactive protein blood test is also used as an indicator of inflammation. Since many inflammatory and autoimmune diseases can produce abnormal rheumatoid factor, citrulline antibody, antinuclear antibody, sedimentation rate and C-reactive protein results, these tests alone cannot be used to diagnose RA.

$\mathrm{RA}$ is difficult to diagnose early since patients in the very early stages of the disease may present without apparent joint swelling. The doctor can use imaging techniques such as High Resolution Ultra Sound (HRUS) and Magnetic Resonance Imaging (MRI) to reveal inflammation that is not apparent by the classical examination technique of palpation. Radiography (X-ray) cannot detect soft tissue inflammation, but can provide information with regards to bone destruction and joint deformation at later stages of the RA disease (Bohndorf 1996, 399-407). The radiography image will show an erosive pattern in general. The figure below demonstrates more specifically the radiographic features of an RA joint. 


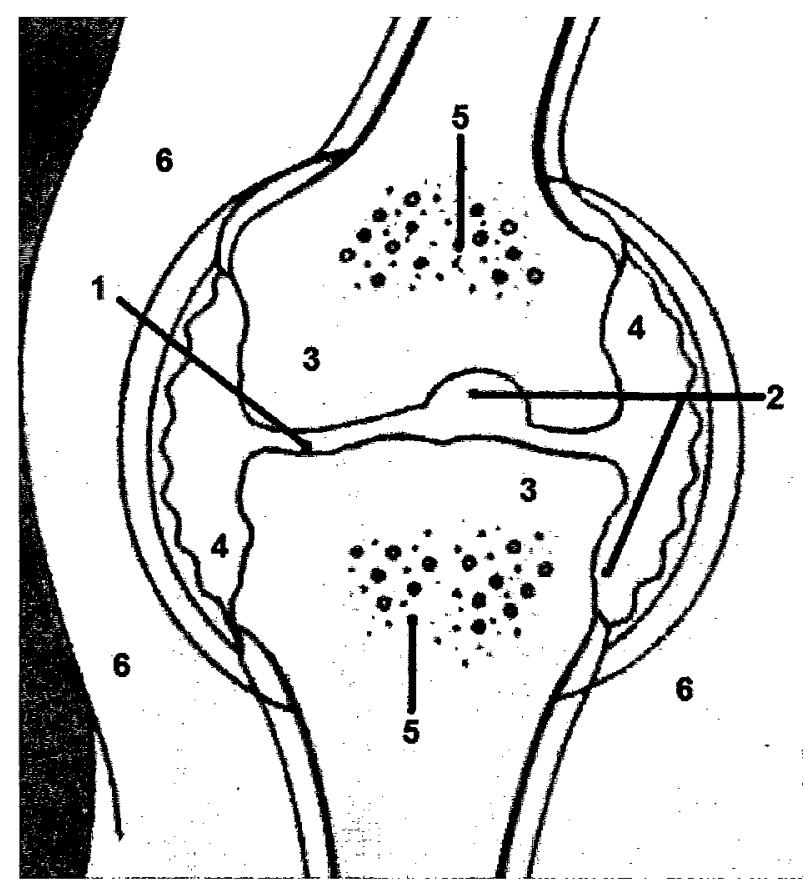

Figure 1-6: Radiographic Features of an RA Joint

In the above figure we can identify the following progressive RA features:

1- Diffuse joint space narrowing

2- Marginal or central erosions

3- Minimal subchondral sclerosis

4- Joint effusion (fluid build-up)

5- Osteopenia (lower bone density)

6- Periarticular soft tissue swelling

The diagnosis of RA is therefore based on the history of the patient's symptoms, the distribution of the inflamed joints, the serum results of the blood tests and the imaging findings. In 1987, the American College of Rheumatology developed revised criteria for the diagnosis of RA. These criteria demonstrate a 
sensitivity of $91-94 \%$ and a specificity of $89 \%$ when used to classify patients with

RA compared with control subjects with rheumatic diseases other than RA.

Table 1-1: American College of Rheumatology's Criteria for the Classification of Rheumatoid Arthritis (Arnett 1998, 315-324)

\begin{tabular}{|l|l|l|}
\hline \multicolumn{2}{|l|}{ Criterion } & Definition \\
\hline 1 & Morning Stiffness & $\begin{array}{l}\text { Morning stiffness in and around the joints, lasting at least 1 hour before } \\
\text { maximal improvement }\end{array}$ \\
\hline 2 & $\begin{array}{l}\text { Arthritis of } 3 \text { or more } \\
\text { joint areas }\end{array}$ & $\begin{array}{l}\text { At least } 3 \text { joint areas simultaneously have had soft tissue swelling or fluid } \\
\text { (not bony overgrowth alone) observed by a physician. The 14 possible } \\
\text { areas are right or left PIP, MCP, wrist, elbow, knee, ankle, and MTP joints }\end{array}$ \\
\hline 3 & $\begin{array}{l}\text { Arthritis of hand } \\
\text { joints }\end{array}$ & At least 1 area swollen (as defined above) in wrist, MCP, or PIP joint \\
\hline 4 & Symmetric arthritis & $\begin{array}{l}\text { Simultaneous involvement of the same joint areas (as defined in 2) on both } \\
\text { sides of the body (bilateral involvement of PIPs, MCPs, or MTPs is } \\
\text { acceptable without absolute symmetry) }\end{array}$ \\
\hline 5 & Rheumatoid nodules & $\begin{array}{l}\text { Subcutaneous nodules, over bony prominences, or extensor surfaces, or in } \\
\text { juxtaarticular regions, observed by a physician }\end{array}$ \\
\hline 6 & $\begin{array}{l}\text { Serum rheumatoid } \\
\text { factor }\end{array}$ & $\begin{array}{l}\text { Demonstration of abnormal amounts of serum rheumatoid factor by any } \\
\text { method for which the result has been positive in <5\% of normal control } \\
\text { subjects }\end{array}$ \\
\hline 7 & $\begin{array}{l}\text { Radiographic } \\
\text { changes }\end{array}$ & $\begin{array}{l}\text { Radiographic changes typical of rheumatoid arthritis on posteroanterior } \\
\text { hand and wrist radiographs, which must include erosions or unequivocal } \\
\text { bony decalcification localized in or most marked adjacent to the involved } \\
\text { joints (osteoarthritis changes alone do not qualify) }\end{array}$ \\
\hline
\end{tabular}

For classification purposes, patient are diagnosed with RA if they satisfy at least 4 of these 7 criteria. Particularly, criteria 1 through 4 must have been present for at least 6 weeks (Arnett 1998, 315-324).

The statement of the problem will compare all the RA diagnosis methods described above to the TIR imaging diagnosis tool for RA. No other tool is at 
once safe, non-ioninzing, inexpensive, non-invasive and capable of examining multiple joints per image in a reasonable scanning time period.

\subsection{Treatment and Therapy of Rheumatoid Arthritis}

RA has no known cure. The objective of RA treatment is to diminish joint inflammation, to reduce the pain and the symptoms of the patient, to maximize joint function and to prevent the destruction and deformation of the joint.

There is consensus among doctors that early diagnosis of RA and initiation of aggressive therapy early in the course of the disease can greatly diminish RA progression and in some patients could even lead to drug free remission. It is therefore important to diagnose the disease and to intervene in its very early stages to have a better prognosis (Bykerk 2009).

Optimal treatment of RA involves a combination of patient education, rest, joint-strengthening exercises, joint protection, and medications. Patients are treated according to their disease activity, the type of joints that are inflicted, their age, their overall health and their occupation/lifestyle. When a combination of these approaches and medications are ineffective, some patients with severe joint deformity may require surgery (Bernard 2003).

\subsubsection{First line medications}

Nonsteroidal anti-inflammatory drugs (NSAIDs) such as acetylsalicylate (Aspirin) and ibuprofen (advil, motrin) are used to reduce tissue inflammation, pain and swelling (O'Dell 2008, chapter 16). Patients respond differently to different NSAID medications; therefore, the doctor usually tries several NSAID 
drugs before identifying the most effective drug with the least of side effects. These include stomach upset, abdominal pain, ulcers and gastrointestinal bleeding.

During flare periods or when NSAIDs are not effective at treating the disease, corticosteroid medications are given orally or injected directly into the joint (O'Dell 2008, chapter 16). These are more potent in reducing inflammation and maximizing joint function, but can have serious side effects which include weight gain, facial puffiness, thinning of the skin and bone, easy bruising, cataracts, risk of infection, muscle wasting, and destruction of large joints. In addition, discontinuing the treatment of corticosteroids abruptly can lead to flares or other symptoms of withdrawal.

\subsubsection{Second line medications}

First line medications reduce joint inflammation and pain; however, they do not directly prevent joint destruction or deformity. To prevent progressive damage to cartilage, bone, ligaments and neighboring soft tissues diseasemodifying anti-rheumatic drugs (DMARDs) are used (American College of Rheumatology Subcommittee on Rheumatoid Arthritis Guidelines 2002, 328346). These are slow-acting medications taken over long periods of time at varying doses and may take weeks to months to become effective. The goal of DMARDs is to promote remission and consequently directly impede the progression of joint destruction and deformity. A combination of second line medication can be used, and like the first line medication, the doctor may need to 
try different drugs before the treatment is optimal. Some examples of DMARDs are hydroxychloroquine (Plaquenil) and sulfasalazine (Azulfidine).

Immunosuppressive medications suppress the body's immune system and can be used for treating RA. Immunosuppressive drugs are usually given to patients with aggressive RA or with serious complications of inflammation because of its potentially serious side effects. Methotraxate is a popular immunosuppressive medication used as an initial second line drug. It is important to note that immunosuppressive medications can reduce bone marrow function and cause anemia, low white cell count and low platelet counts. Specifically, methotraxate can sometimes lead to cirrhosis and allergic reactions in the lung. Cyclosporine, another immunosuppressive drug, can cause kidney damage and high blood pressure. These side effects are the reason these drugs are prescribed in low doses and in combination with anti-inflammatory medications (O'Dell 2008, chapter 16).

The efficacy of first and second line treatments can be detected using IR thermography (Ring 1974, 353). This information will be presented in the chapter 2 literature review of this thesis. Furthermore, the awareness of the medication pathways will facilitate the analysis and discussion in chapter 5 .

\subsection{Statement of the Problem}

It is critical to diagnose a patient with RA very early in the course of the disease, first to prevent the non-reversible and permanent damage to the synovial joint, and second to allow the doctor to intervene early with an 
aggressive therapy. The prognosis of a diagnosed RA patient is better when discovered and treated early (Bykerk 2009). For that reason, the doctor's goal is to diagnose the disease as early as possible, even before the first clinical signs are apparent - pain and swelling. Imaging techniques can be a solution to this difficulty allowing the physician to visualize the joints in further detail to make an early diagnosis for treatment.

As seen previously, diagnosis is particularly difficult early on in the disease. During this stage, the patient may not present with the apparent RA symptoms - joint swelling, redness, pain, warmth, tenderness. Therefore, it is important to be able to combine the classical examination technique of palpation - which may not detect RA very early on - with imaging techniques that are able to visualize the bone and the soft tissues of the joint; more particularly the synovial membrane. It is also important to clearly identify the distribution of the affected joints to distinguish between RA and other arthritic diseases, such as osteoarthritis. Imaging techniques can help determine the state of the synovial membrane and the distribution of the involved joints. Furthermore, imaging techniques can eliminate diagnostic inter-physician and intra-physician variations. The clinical evaluation - history and physical exam - is subject to observer variations and is limited to the expertise of the individual performing the evaluation. Doctors therefore have an interest in using imaging tools to help facilitate the diagnosis of RA (as verbally stated by the co-investigator of this study - Dr. Jacob Karsh, Rheumatologist at the Ottawa Hospital - Riverside Campus) (Dr. Jacob Karsh, Personal Communication, September $22^{\text {nd }} 2009$ ). 
The diagnostic serum tests explained above cannot be used alone to identify RA. No single marker has been shown to predict RA outcome well. Therefore imaging procedures still remain the best diagnostic tool used by physicians to identify and assess RA.

Magnetic Resonance Imaging (MRI) and High Resolution Ultrasound (HRUS) are the two imaging tools that are currently used by doctors to diagnose RA. MRI is an imaging technique using radio frequency pulses and a powerful magnetic field to visualize the internal structures of the body - bone, organs, soft tissues, etc. It produces very high quality images with a great contrast between different soft tissues (Sugimoto 2000, 569-575). However, the MRI imaging procedure requires the patient to remain perfectly still while the images are being recorded. MRI exams include multiple sequences that last several minutes each. The entire exam duration is time consuming ranging from 15 minutes up to 45 minutes. Patients with RA, who may be experiencing severe pain or discomfort, may find this procedure very long and difficult. There are also some restraining contreindications to MRI: claustrophobia or anxiety, internal defibrillators or pacemakers and cochlear ear implants. Furthermore, MRI procedures are expensive and the wait times are long. In Ontario, the average patient waits 120 days before having an MRI imaging session (Government of Ontario 2009). High Resolution Ultra Sound (HRUS) is an imaging technique that emits high frequency sound waves into the body and creates an image based on the measurements of the echoes returned from the inner tissues. HRUS can view soft tissue thus is a tool that is able to visualize swelling and inflammation. It is 
also less expensive and more available than MRI; however, HRUS produces lower quality images than MRI. According to Dr. Jacob Karsh, there are ongoing investigations examining the diagnostic utility of HRUS since it is limited to a fewer number of joints (Dr. Jacob Karsh, Personal Communication, September $22^{\text {nd }} 2009$ ). It is for these reasons that there is a need of an imaging method that is inexpensive, non-invasive, not limited to only imaging a few joints and that is capable of examining multiple joints at a time for the assessment of RA.

\subsection{Thesis Objectives}

The main objective of this thesis is to explore IR imaging as a possible inexpensive non-invasive method to diagnose and treat RA. Thermal IR (TIR) systems would make the diagnosis process more efficient since it would reduce the length of the imaging procedure given that some images would be able to examine multiple joints at a time. For example, one single IR image of the hand can at once evaluate 15 joints - the 5 MCPs, 5 PIPs, 5 DIPs joints and the wrist. Thermal IR (TIR) imaging can also quantify the inflammation and severity of the affected joint to assist physicians in treating their RA patients.

The first objective of this thesis is to determine if it is possible to discriminate between the control group and the RA group. The second objective is to identify the joints to examine in a clinical setting that best determine if a participant has or does not have RA. The third objective of this thesis is to identify the best mathematical calculation of the data to assess the presence or absence of RA. The fourth objective is to determine if thermal IR images can be 
used to classify the RA patients into three severity groups - high degree of RA, moderate degree of RA and low degree of RA. The final objective of this thesis is to observe the effect of age on the thermal IR measurements.

\subsection{Thesis Outline}

The introduction has covered the thesis motivation, the statement of the problem, a detailed background on rheumatoid arthritis, and the thesis objectives and outline.

Chapter 2 presents a literature review beginning with thermal infrared imaging. This section covers the electromagnetic spectrum, blackbody radiation and emissivity, Planck's law and Wien's displacement law, and Stefan Boltzmann's law. The chapter goes on to explore the past research in infrared thermography. Afterwards, a section on clinical infrared thermography gives an overview on general medical applications and rheumatoid arthritis applications of thermal infrared technology.

Chapter 3 discusses the methodology used in this research thesis. It covers the extensive ethics review process that was completed to begin this project, followed by the recruitment process of the participants. A description of the infrared system and the setup that was used for the imaging procedure is given. The chapter continues with the collection of data methodology which covers the thermal infrared protocols and recording procedures. It concludes with an explanation of the analysis of data. 
Chapter 4 begins by displaying the resulting datasets derived from the raw data measurements extracted from the thermal infrared images. The chapter goes on to apply the Lilliefors Normality Test to observe if the modified raw dataset distributions (called matrices within this thesis) are Gaussian. Then hypothesis tests are done to show the statistical significance of the results to see if each thesis objective can be met.

Chapter 5 states the contributions to knowledge of this thesis work. It will end with some proposed future work. 


\section{CHAPTER 2}

\section{LITTERATURE REVIEW}

This chapter presents a literature review beginning with thermal infrared imaging - the electromagnetic spectrum, blackbody radiation and emissivity, Planck's law and Wien's displacement law, and Stefan Boltzmann's law. The chapter goes on to explore the past research in infrared thermography. Afterwards, a section on clinical infrared thermography gives an overview on general medical applications and rheumatoid arthritis applications of thermal infrared technology.

\subsection{Thermal Infrared Imaging}

\subsubsection{Electromagnetic Spectrum}

The electromagnetic spectrum is divided into wavelength bands. Thermographic cameras detect radiation in the infrared spectral range. The infrared band is subdivided into four sub-bands: near infrared $(0.75$ to $3 \mu \mathrm{m})$, middle infrared ( 3 to $6 \mu \mathrm{m}$ ), far infrared ( 6 to $15 \mu \mathrm{m}$ ) and extreme infrared (15 to $100 \mu \mathrm{m})$ (FLIR Systems 2004). 


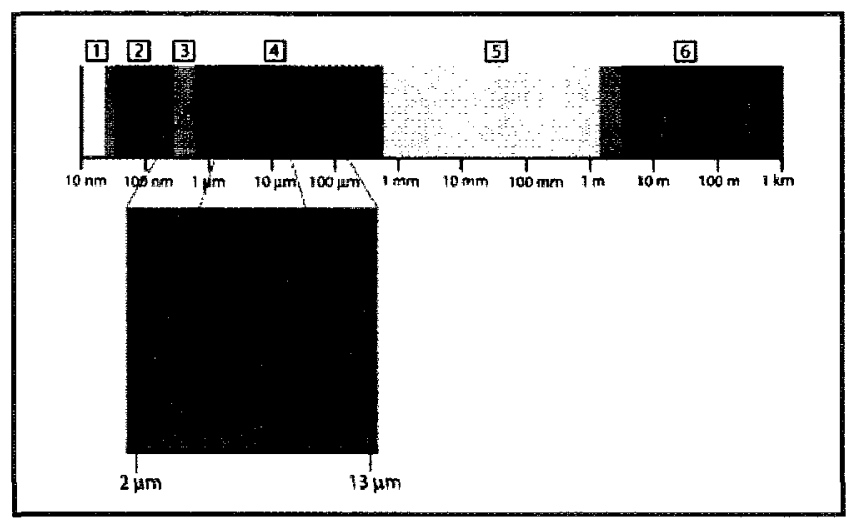

Figure 2-1: Electromagnetic Spectrum (FLIR Systems, 2004)

Military researchers developed this technology for the purpose of using thermal IR cameras for night vision and human detection in war situations. Consequently, most thermal IR cameras function in the far infrared range since the infrared signal at these wavelengths travel best through smoke, smog, dust, humidity and other possible distortion factors in the environment.

\subsubsection{Blackbody Radiation and Emissivity}

All surfaces heated at a temperature above zero degrees Kelvin ( ${ }^{\circ} \mathrm{K}$ ) or 273 degrees Celsius $\left({ }^{\circ} \mathrm{C}\right)$ emit electromagnetic radiation. A blackbody is an object that can perfectly absorb all radiation at any wavelength and that can equally emit all possible wavelengths of energy (Kirchhoff's law). The emissivity of a material is a measure of its ability to absorb and radiate energy. It is the ratio between the power radiated by the material and the power radiated by a blackbody at the same temperature as seen in Equation 2.1.

$$
\varepsilon_{0}(T)=\frac{W_{0}(\lambda, T)}{W_{b}(\lambda, T)}
$$


A blackbody has an emissivity of $\varepsilon=1$. The emissivity of an object depends on the temperature, wavelength and direction. However, it is possible to consider it a constant within a determined temperature range, bandwidth and cone of direction (Wolfe 1965, Hudson 1969 and Gaussorgues 1994).

Treated surfaces have an emissivity ranging from 0.1 to 0.95 . For instance, an oxidized or painted surface has an emissivity of approximately 0.90 whereas a highly polished surface, such as a mirror, has an emissivity below 0.1 . Human skin has an emissivity resembling that of a blackbody - estimated between $\varepsilon=0.97$ and $\varepsilon=0.98-$ within the $8-14 \mu \mathrm{m}$ range (the far infrared range). In the 3-5 $\mu \mathrm{m}$ IR region (the middle infrared range), the emissivity of the skin decreases and is more prone to environmental artefacts (Anbar 1994). Therefore, measurements of the skin using thermal IR techniques should be performed in the 8-14 $\mu \mathrm{m}$ range to achieve a higher accuracy of results.

\subsubsection{Planck's Law and Wien's Displacement Law}

The radiation emitted by a blackbody can be described through Planck's law. The following equation represents the spectral distribution of the radiation from a blackbody (FLIR Systems, 2004):

$$
W_{\lambda b}=\frac{2 \pi h c^{3}}{\lambda^{5}\left(e^{\frac{h c}{\lambda k T}}-1\right)} \times 10^{-6}\left[\text { Watt } / m^{2} \mu m\right]
$$

where $W_{\lambda b}$ is the blackbody spectral radiant emittance at wavelength $\lambda, \mathrm{c}$ is the velocity of light $\left(3 \times 10^{8} \mathrm{~m} / \mathrm{s}\right), h$ is the Planck's constant $\left(6.626 \times 10^{-34}\right.$ Joule sec.), $k$ is the Boltzmann's constant $\left(1.380 \times 10^{-23}\right.$ Joule/K), $T$ is the absolute 
temperature $(\mathrm{K})$ of a blackbody and $\lambda$ is the wavelength $(\mu \mathrm{m})$. Planck's radiation law describes how, as the temperature increases, the peaks of the radiated spectrum move towards shorter wavelengths and the radiation power emitted increases. This effect is seen in the figure below, which plots the spectral radiant emittance as a function of wavelength for various temperatures.

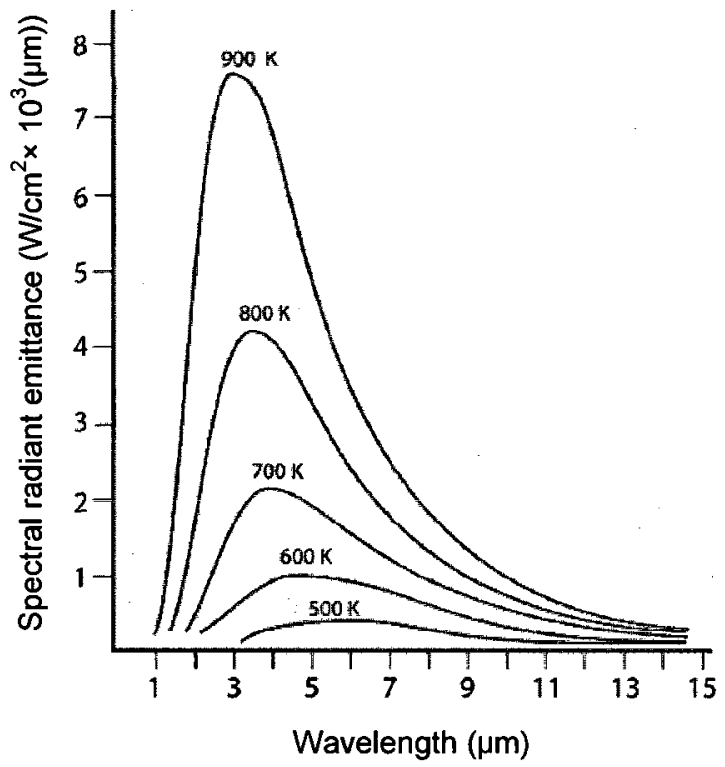

Figure 2-2: Emissive Power of a Blackbody at Different Temperatures and Wavelenghts According to Planck's Radiation Law (FLIR Systems, 2004)

The location of the peaks can be expressed by differentiating Planck's law with regards to $\lambda$ and finding the maximum. This is known as Wien's law (FLIR Systems 2004):

$$
\lambda_{\max }=\frac{2898}{T}[\mu \mathrm{m}]
$$

where $\lambda_{\max }$ is the maximum emissive power and $\mathrm{T}$ is the temperature (in ${ }^{\circ} \mathrm{K}$ ). Wien's formula describes the common observation that colors vary from red to 
orange to yellow as the temperature increases. Consequently, at room temperature $\left(300^{\circ} \mathrm{K}\right)$ the maximum emissive power is attained at a wavelength of $9.7 \mu \mathrm{m}$, in the far infrared region of the electromagnetic spectrum. This correlates with what was stated earlier - that the body's radiated power is significant in the 8-14 $\mu \mathrm{m}$, the far-infrared spectrum. The figure below shows the Planckian curves from $100 \mathrm{~K}$ to $1000 \mathrm{~K}$. The dotted line represents the maximum radiant emittance at each temperature according to Wien's displacement law.

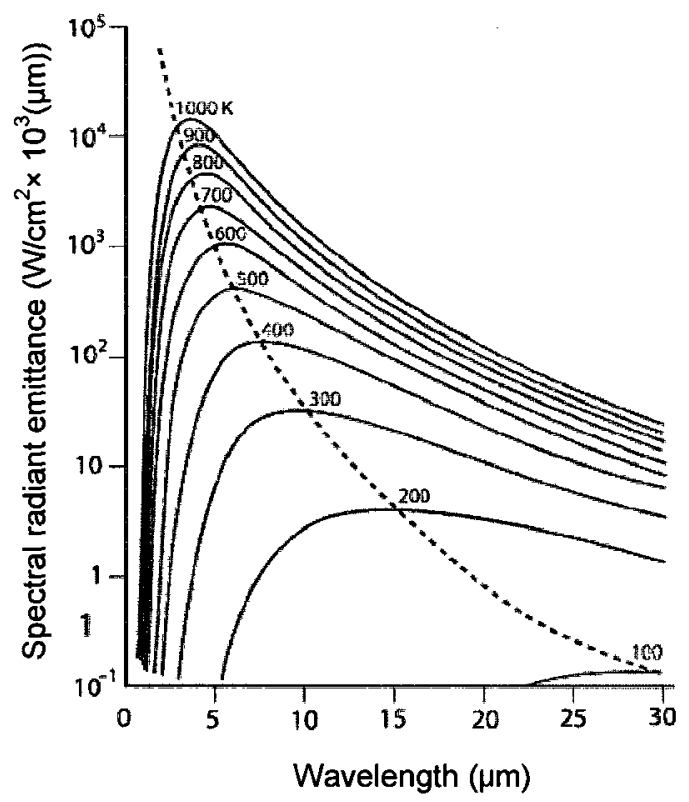

Figure 2-3: Planckian Curves Plotted on Semilog Scale (FLIR Systems, 2004)

\subsubsection{Stefan Boltzmann's Law}

The Stefan-Boltzmann's law represents the total emissive power or the total radiant emittance $\left(W_{b}\right)$ of a blackbody. This formula is obtained by integrating Planck's equation from $\lambda=0$ to $\lambda=\infty$ and is as follows (FLIR Systems 2004): 


$$
W_{b}=\sigma T^{4}\left[\text { Watt } / m^{2}\right]
$$

where $\sigma=\frac{2 \pi^{5} k^{4}}{15 c^{2} h^{3}} \approx 5.670 \times 10^{-8}\left[\mathrm{Watt} / \mathrm{m}^{2} \mathrm{~K}^{4}\right]$ is the Stefan-Bolztmann's constant.

Graphically, $W_{b}$ is the area below the Planck curve at a particular temperature. From Equation 2.4, the power emitted by the human body at room temperature $\left(300^{\circ} \mathrm{K}\right)$ with a surface area of approximately $2 \mathrm{~m}^{2}$ is $1 \mathrm{~kW}$.

The amount of energy that is reflected by an object is determined by the reflectivity coefficient $\rho_{\lambda}$. The reflectivity is calculated as follows:

$$
\rho_{\lambda}=1-\varepsilon_{\lambda}
$$

A blackbody has a reflectivity of 0 , since all the radiation is absorbed and emitted. When a gradient of temperature is applied to a blackbody and if only radiative heat transfer is considered, the Stefan-Boltzmann's law becomes:

$$
W_{b}=\sigma\left(T_{b}{ }^{4}-T_{s}^{4}\right)\left[\text { Watt } / m^{2}\right]
$$

where $T_{b}$ is the temperature of the body (in Kelvin) and $T_{s}$ is the temperature of the surroundings (in Kelvin).

From Planck's law (equation 2.2) and the emissivity equation (equation 2.1) it is possible to directly calculate the temperature of a body knowing the measured emitted power of that body at a specific wavelength. However, when an object has an emissivity less than unity the reflectivity of that body must be taken into account when calculating the temperature of that object from the measured total energy emitted from that body. For this reason, it is desirable to measure objects that have an emissivity as close to unity as possible for a higher 
accuracy of temperature measurement (Anbar 1994). Since the skin has an emissivity close to that of a blackbody, the adjustment needed to account for the reflectivity of the skin is negligible, especially when the temperature of the environment is lower than that of the skin and the angle of viewing of the measurement is less than $\pi / 4$ (Jones $1998,1019-1027$ ). It is important to note that in a clinical setting, it is possible to assume that the reflectivity has no effect on the direct link between the measured energy radiated by the object and the temperature of that object since the environment's temperature is controlled to be lower than that of the skin and the viewing angle is ensured to be less than $\pi / 4$.

\subsection{Past Research in Infrared Thermography}

Over the past 50 years, Thermal Infrared (TIR) imaging has been applied to many fields such as military, veterinary and electronic maintenance applications (Ernisse 1997, 2593-2603 and Huang 2005, 3281-3288). From the 1960 's to the 1980 's, medical thermal imaging applications were investigated.

Promising results were found in research such as pain assessment, breast cancer and arthritis (Collins 1974, 113-115; Aubry-Frize 1981 and Gautherie 1983, 74-92). The major limitations found in the early work included physical restrictions of early systems (large size, heavy weight, cooling requirements), subjectivity of the thermographic interpretations, limited power of computers for the imaging analysis, lower sensitivity and high cost of IR systems. These issues prevented the widespread applications of TIR imaging in the medical field until recently. The renewed interest in clinical IR thermography is due to the 
advances in IR technology, computers for analysis and standard protocols for examination. New IR systems are physically compact, cost less, have a higher thermal and spatial resolution, and have faster frame and processing rates. Today's computers also have faster processing speeds and high definition monitors for the IR analysis. This combination allows a higher quality of digital IR imaging for medical applications. Furthermore, since the mid 1990's standards for medical IR thermography examinations have been agreed upon (Ring 1995, 1697-1698). On the other hand, the issue of interpreting the higher quality IR images remains. Clinical experts trained in the interpretation of thermograms are the current standard in medical applications. However, the ability of the eye to identify the subtle temperature variations and recognise digital distribution patterns is not very good. It has also been shown that the use of pseudo-colours to improve human interpretation is not a good solution given that humans are subject to psychological effect of hot and cold colours (Anbar 1991, 479-484). Our group has introduced a method to improve IR interpretation by applying recovering processes for the contour of images to reduce noise and improve the low contrast. We also developed a segmentation process to identify abnormal regions on the IR surface of the body (Herry 2007, Herry 2008). Our group intends to incorporate this approach to the clinical application of assessing RA.

\subsection{Clinical Infrared Thermography}

It is possible to define clinical infrared thermography or thermal infrared imaging as the recording of the temperature distribution of the human body (or 
body areas) using infrared radiation emitted by the surface of that body - for example the skin (Jones 1998, 1019-1027). TIR images can provide valuable information on the physiology of the human body, since a major part of the energy radiated by the human body goes through the skin and can be detected by thermographic sensors in the infrared range of the electromagnetic spectrum (Houdas 1982). Some examples of information that can be analysed are neurological activity, vascular flow and distribution, rheumatic activity and abnormal growths in oncology provided that the temperature changes can be seen on the surface of the skin. Thermal IR images can provide a general indication of location and/or sources of pathologies of anatomical nature (Anbar 1991, 479-484). Using thermocouples, Horvath and Hollander have shown that the intra-articular temperature of RA patients can be used as a guide to the severity of inflammation (Horvath 1949, 469-473). Bacon et al. have demonstrated that measurement of mean skin temperature could be used as a measure of disease activity (Bacon 1976, 51-65). Furthermore, Ring et al. and Collins and Cosh have shown that variations in skin temperature over areas of chronic inflammation correctly reflect the chemical and cellular changes in rheumatoid arthritis (Ring 1970, 337 and Collins 1970, 386).

Many studies, such as Goodman et al. and Uematsu et al., have shown that since the skin temperature distribution of a normal healthy individual exhibits a contralateral symmetry, it is possible to identify some abnormalities when the temperature distribution shows asymmetrical patterns (Goodman 1986, 195-202; Uematsu 1985 and Uematsu 1988, 552-555). However, in some pathological 
diseases - such as rheumatoid arthritis - this might not always be helpful because the thermal dysfunctions are usually exhibited bilaterally. In cases where the disease is present bilaterally, the expert interpreting the thermographic image must find other signs of abnormalities in the temperature distribution and its patterns (Hooshmand 2001, 53-65). Interpreting the IR image is one of the most difficult processes in IR applications since the expert interpreter needs to understand the complexity of the pathology and the underlying processes that cause the thermal distribution dysfunction. (It is for this reason that Chapter 1 covers detailed information on the pathophysiology of RA.) Thus, the sensitivity and the specificity of the interpretation technique is greatly dependant on the pathological application of the IR technology. ${ }^{1}$

\subsubsection{General Medical Applications of TIR Technology}

The early literature on medical application of TIR technology focused on interpreting thermographic images and identifying abnormal thermal variations on the surface of the skin. This was done via visual evaluation of grey-level and/or pseudocoloured thermal images using isothermal functions, by identifying hot or cold regions within a Region of Interest (ROI) and by asymmetrical analysis (Aubry-Frize 1982, 82-83; Hooshmand 2001, 53-65; Hubbard 1985, 26-35;

Poussart 1988; LeRoy 1985 4-13; Kim 1995, 1699-1700; Goldberg 1986; Thomas 1990, 268-273; Ellis 1989). Head and Lipari studied the thermal

${ }^{1}$ The sensitivity is defined as sens. $=\frac{T P}{T P+F N}$ and the specificity as spec. $=\frac{T N}{T N+F P}$ where TP, TN, FP and FN are true positive, true negative, false positive and false negative respectively. 
distribution on the surface of breasts for breast cancer risk assessment. They analysed the images by manually selecting a large ROI along the contour of the entire breast using anatomical reference points and splitting each large ROI into four smaller ROls (Head 1997, 681-684 and Lipari 1997, 673-676). Complex methods of boundary detection have been studied and successfully implemented for breast segmentation to allow automation $(\mathrm{Ng} \mathrm{2001,12-16}$ and Scales 2004, 1737-1740). Other techniques of ROI automation have been attempted using military Automated Target Recognition (ATR) techniques (Irvine 2002, 31-40). The major obstacle in implementing military ATR to the clinical setting is the nature of the target of study. In a military setting, the shape of the ROI is already known, whereas in the clinical setting, the shape of the region of pain/tumour/joint is not always the same. The shape and size of the diseased region can vary from patient to patient and even within one patient over time.

Once the region of interest is selected, statistical analysis is performed on the selected ROI. This analysis mostly includes thermal detection of maximum and minimum temperature regions and first-order statistics (mean, median, standard deviation). Histograms are also used to graphically represent the temperature distribution in discrete time. Studies were also done to assess the effect of scaling on temperature variations and accuracy by varying the size of the ROI (Goodman 1986, 195-202 and Lipari 1997, 673-676).

Moreover, to ensure the accuracy of medical TIR readings it is important to implement the correct protocols and standards - control of the environment (temperature, reflectivity, humidity, etc.) and control of thermal characteristics of 
the skin (no lotions or creams, no extreme exercise, no hot/cold beverages prior to the examination, etc.) (Ring 1995, 1697-1698; Jones 1998, 1019-1027 and Herry 2004). The intensities of IR images depend on the object's temperature and surface properties and on the surface's orientation and wavelength. These properties are not always uniform across one surface where the temperature is uniform. As mentioned previously, environmental factors at the time of imaging greatly influence the IR images that are collected; however, in a clinical setting, these factors can be managed and controlled. Furthermore, controlling the room at ambient temperature (approximately $20^{\circ} \mathrm{C}$ ) increases the sensitivity of the skin's surface temperature to subcutaneous vascular flow when a cooling period is implemented (Stolwijk 1976, 40-51). Thus, it is recommended that prior to the TIR imaging procedures, the subject remains in the room at ambient temperature for a cooling period in order that the skin's radiating energy best reflects the blood flow and other cellular activity under the skin.

\subsubsection{RA Applications of TIR Technology}

Collins and Ring used a Thermal Index $(\mathrm{TI})^{2}$ calculation based on the isothermal temperature difference to quantify the measurement of synovitis (Collins 1974, 113 and Ring 1974, 353). The TI they introduced reflects the mean temperature in a determined ROI. Collins and Ring were able to show that

2 The Thermographic Index is measured from the expression: $\frac{\sum(\Delta t \times a)}{A}$; where $\Delta t$ is the difference between the measured isothermal temperature and the lowest common isotherm recorded, $a$ is the area occupied by isotherm $\left(\mathrm{cm}^{2}\right)$ and $A$ is the total area of the thermogram $\left(\mathrm{cm}^{2}\right)$. 
IR thermograms of the knees, hands and feet can be used to identify patients with RA and as an assessment tool for the disease. They showed that the TI decreased with treatment and that the $\mathrm{TI}$ increased during a flare episode of RA. Using a larger sample group and the t-test, Bird, Ring and Bacon's publication confirmed Collins and Ring's findings that thermography can be used to assess inflammation of the synovial membrane and to evaluate the effectiveness of an RA treatment (Bird 1979, 36-39). They studied the effect of intra-articular injection drugs on the state of RA knees and concluded that the TIR images showed improvements in the patients after the treatment was initiated.

In 1983, Salisbury et al. suggested a new method of interpreting the IR information to detect RA abnormalities in the knee, wrist, elbow and ankle joints (Salisbury 1983, Salisbury). He demonstrated that the quantification of synovitis by TIR imaging should be based on dysfunctional thermal patterns rather than by indexes, such as the $\mathrm{TI}$, that are based on absolute skin temperature values. The skin's surface temperature is greatly affected by many factors such as metabolic rate, consumption of very hot/cold beverages, calorie intake, emotional state, circadian variation, physical activity, environment temperature and humidity, etc. (Reinberg 1976, 128-139) Salisbury et al. therefore introduced a Heat Distribution Index (HDI) that would be a representation of pattern and spread of the temperature distribution over the surface of a joint. They went on to prove that the HDI correlated better with joint inflammation and synovitis than Collins and Ring's $\mathrm{TI}$. The $\mathrm{TI}$ proved to be less significant in identifying the presence of inflammation and was even less significant in identifying 
inflammation in afternoon TIR readings. The $\mathrm{TI}$ is more variable and less reproducible than the $\mathrm{HDI}$, since it is greatly affected by the patient's circadian rhythm and the environment in which the images are taken. The HDI was able to identify a normal thermal distribution in the absence of inflammation in the knee, ankle, wrist and elbow joints. Alternatively, inflammation of the synovial membrane was repeatedly detected by the HDI analysis and these results were not affected by the time of day of the TIR readings. Salisbury et al.'s HDI is calculated based on the relative frequency distribution of the thermogram via a 256 grey level TIR image. A ROI of interest is delimited to cover the joint to be analysed. A pixel count is made at each grey level within the ROI and each resulting number is divided by the number of pixels in the ROI. Normal joints present a narrower relative frequency distribution (lower standard deviation) than a diseased joint. Salisbury et al. also added that the distribution of heat over a normal joint results in a positive temperature gradient from the centre to the periphery of the joint. For example, when observing the lateral view of the knee, the temperature increases from the knee's patella (or knee cap) to the back of the knee joint periphery - the patella having the lowest temperature. They also noted that normal joints do not have localised areas of increased temperature hot spots. An abnormal joint with synovitis can therefore be described as a joint presenting with a negative thermal gradient with localized hot spots within the joint's ROI.

Devereaux et al. supported Salisbury et al's work by stating that the HDI significantly correlates with other RA evaluation methods - the Ritchie articular 
index (a graded assessment of 26 joint regions to assess tenderness plus a 44 joint count to assess swelling), grip strength, morning stiffness, erythrocyte sedimentation rate and pain score (Devereaux 1985, 434-437). They confirmed, using the product moment coefficient correlation test, that the thermographic HDI is a sensitive, reproducible, quantifiable method of analysis that gives results comparable to the current methods of assessment. In addition to its high correlation, TIR imaging using the HDI technique avoids complications that the current methods do not, such as invasiveness, interobserver variability, high cost, circadian variation, etc. Devereaux et al. also demonstrated that the HDI can be used to confirm if the disease is more severe or better controlled by observing patient's progress over time.

De Silva et al. compared the use of TIR imaging (using the HDI analysis procedure) to the clinical assessment and to the radioisotopic imaging methods of evaluating RA (De Silva 1986, 277-280). Using Spearman correlation coefficients, they found a high correlation between the HDI and the radioisotopic imaging method in rheumatoid arthritic knees. The HDI also significantly correlated with every aspect of the clinical assessment - swelling, tenderness, morning stiffness, joint score - except for pain.

Other research, such as llowite et al's, went on to validate the above findings and further confirm that the thermographic HDI is the best method to assess inflammation of the synovial membrane for RA diagnosis (llowite 1992, 343-346). 
In this thesis we analyse the $2^{\text {nd }}$ and the $3^{\text {rd }}$ MCPs, the $2^{\text {nd }}$ and the $3^{\text {rd }}$ PIPs, the wrists and the knees based on the temperature distribution methodology introduced by Salisbury and supported by many others. The temperature distribution pattern is analysed to see if abnormal localized regions of increased or decreased temperatures can be used to classify participants with RA. Moreover, histogram functions and the relative frequency of hot/cold regions were explored as another method of distinguishing patients from control subjects. 


\section{CHAPTER 3}

\section{METHODOLOGY}

Chapter 3 discusses the methodology used in this research thesis. It covers the extensive ethics review process completed prior to beginning this project, followed by the recruitment process of the participants. A description of the infrared system and the setup that was used for the imaging procedure is given. The chapter continues with the collection of data methodology which covers the thermal infrared protocols and recording procedures. It concludes with an explanation of the analysis of data.

\subsection{Ethics Review}

A pilot research proposal was approved by the Ottawa Hospital Research Ethics Board (OHREB) under the title of "Severity of illness measures in rheumatoid arthritis from multi-spectral imaging". Its principal investigator at the Ottawa Hospital was Dr. Jacob Karsh, a rheumatologist from the Riverside Campus. The co-investigator was Dr. Monique Frize from the Faculty of Engineering and Design at Carleton University. Since the pilot study involved the facilities and the instrumentation at both Carleton University and the University of Ottawa, two more research proposals were submitted and individually approved 
by the Carleton University Research Ethics Committee and the University of Ottawa Research Ethics Board. This thesis is part of the first objective outlined in the proposal submitted: "To perform an exploratory pilot test with patients diagnosed with RA to observe the various degrees of inflammation of patient articulations via heat distribution patterns using infrared thermography." The completion of this study would extend Dr. Frize's Medical Informationtechnologies Research Group (MIRG) at Carleton University in infrared thermography.

\subsection{Recruitment of Participants}

Research participants were patients over the age of 19 years of age of the Ottawa Hospital-Riverside Campus who were diagnosed with RA. They were recruited by their healthcare specialist - Dr. Jacob Karsh - who informed them of the study and asked them if they were interested in participating. Patients of both sexes and of any age were asked to participate in the research study. The exclusion criteria were patients who have joint pain for reasons other than RA; patients who have recently suffered bruising or trauma of the affected joints; and patients with joint sepsis. If patients were interested in the research study, the healthcare specialist provided them with a permission text which they signed to continue with the research process (see Appendix A). This signed permission sheet declared that patients agreed to release their name and contact information to the co-investigators of this study at Carleton University and the 
University of Ottawa. The patients were not given any research incentives to participate in the study, and were not coerced into participating in any other way.

Once the permission text was signed, the co-investigator's student, Cynthia Adéa, contacted the patients individually to confirm their interest and to send them "Research Participant Information and Consent Form" documents (see Appendix B). The patients who consented to the study completed the consent form which permitted the usage and storage of the digital IR images of their limbs and joints. No images of participants' faces were taken and no personal information was shared with Carleton University or the University of Ottawa's research teams.

The control participants were subjects over the age of 19 years old recruited at the University Campuses who have not been diagnosed with RA, who were in good general health and who were without pain or symptoms resembling RA. Control subjects were recruited to establish a range of temperature distribution for normal subjects and to provide a baseline for what is identified as normal. The control participants were not given any research incentives to participate in the study, and were not coerced into participating in any other way. They were given the same information package as the RA patients (see Appendix B) and were asked to sign a consent form which permitted the usage and storage of the digital IR images of their limbs and joints. No images of control subjects' faces were taken and no personal information was shared with Carleton University or the University of Ottawa's research teams. 
Thirteen patients diagnosed with RA were recruited from the Ottawa Hospital-Riverside Campus and eighteen control subjects were recruited from Carleton University, the University of Ottawa and a few from outside the campuses.

\subsection{Collection of Data}

\subsubsection{Specifications of the Infrared Camera}

The ThermoVision A40M Researcher Infrared Camera from FLIR Systems was used to capture the TIR images. This IR camera uses an uncooled microbolometer focal plane array of $320 \times 240$ pixels at a refresh rate of $50 / 60$ $\mathrm{Hz}$. It operates in the $7.5 \mu \mathrm{m}$ to $13 \mu \mathrm{m}$ of the far IR spectral range of the electromagnetic spectrum. The $\mathrm{A} 40 \mathrm{M}$ has a $24 \mathrm{~mm}$ germanium lens with an anti-reflective coating. The germanium lens renders a field of view that is $24^{\circ} \mathrm{x}$ $18^{\circ}$ with a minimum focus distance of $0.3 \mathrm{~m}$. The camera's spatial resolution is $1.3 \mathrm{mrad}$, which means that at a distance of $1 \mathrm{~m}$ each pixel represents a square of $1.3 \mathrm{~mm} \times 1.3 \mathrm{~mm}$. The $A 40 \mathrm{M}$ has a thermal sensitivity of $0.08{ }^{\circ} \mathrm{C}$ at $30^{\circ} \mathrm{C}$ and can detect temperature ranges from $-40{ }^{\circ} \mathrm{C}$ to $+120{ }^{\circ} \mathrm{C}$. The camera was connected to a laptop via a Firewire output connection, thereby capturing sequences of 14 bit digital thermal images at a maximum speed of 60 frames per seconds. 


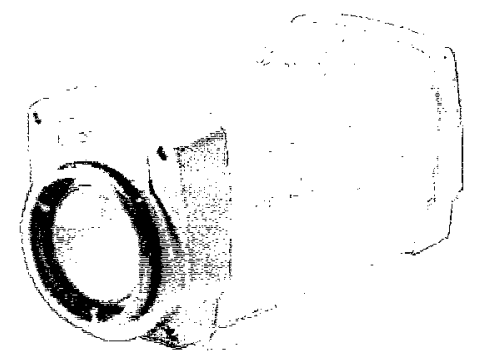

Figure 3-1: A40M Infrared Camera from FLIR Systems FLIR Systems, 2004)

To see the complete specifications of the A40M IR Camera from FLIR Systems turn to Appendix C.

\subsubsection{Infrared Camera and Recording Settings}

The software used during the imaging sessions was the ThermaCAM Researcher Pro 2.8 from FLIR Systems. It is via this software that measurement corrections were inputed by the user - the emissivity, the camera distance, the ambient temperature and the relative humidity. The $\mathrm{A} 40 \mathrm{M}$ radiometric camera outputs emissivity measurements to the ThermaCAM software which converts the measurements into absolute temperatures while taking into consideration all the input corrections.

The camera settings used for this thesis were as follows. The emissivity was kept constant at 0.98 for the skin. The camera distance ranged from $0.5 \mathrm{~m}$ to $1 \mathrm{~m}$ and was manually adjusted for every shot via the settings options of the software. The ambient temperature and relative humidity of the environment were measured for each session and were $23 \pm 1{ }^{\circ} \mathrm{C}$ and $40 \pm 2 \%$ respectively for all the sessions. The image intervals for the recording conditions were set at 2, which means 30 images were taken per second during each recording time. 
The image presentation box was enabled to allow the user to view the images live and manually adjust the camera for the best shot.

Images were recorded in *.seq format for sequences of images and were subsequently exported into Matlab as *.mat files. The *.mat files contained the temperatures of each pixel of the image and information about the environmental settings.

\subsubsection{Medical Thermal Infrared Protocols}

Prior to the imaging session, each participant was given these guidelines to follow:

- Do not consume alcoholic beverages twenty-four hours prior to the session.

- Do not consume hot beverages at least one hour prior to the session.

- Do not wear rings, necklaces, anklets and/or bracelets during the session.

- Do not use talcum powder, lotion, drug or deodorant on the skin on the day of the session.

- Avoid prolonged sun exposure at least one week prior to the session.

- Avoid smoking at least two hours prior to the session.

- Avoid any intense physical exercise at least four hours prior to the session.

- Avoid procedures such as electromyography, acupuncture, myelography, transcutaneous electrical stimulation, hot or cold patches, or any other form of physiotherapy at least twenty-four hours prior to the session.

\subsubsection{Recording Procedures}

The IR thermographic sessions took place in a room without windows to avoid any reflection captured by the camera. There were no shiny or plastic floors and the only lights used were fluorescent lights. The temperature and humidity were measured before and after every session. They were uniform throughout the room and were kept constant throughout the entire session. 
The patients and control subjects were asked to apply the medical thermal infrared protocol prior to the session (as described in the previous section). They were also asked to wear a sleeveless shirt and shorts and to remove their socks and shoes to facilitate the imaging of every articulation.

Before the recording session, the participants were placed on a chair with their arms resting to their sides and their bare feet relaxed on a foam carpet for a cooling down period of 15 minutes. During this time, the participants' surface body temperature was cooled sufficiently in a room remaining constant around $23{ }^{\circ} \mathrm{C}$ to allow the subcutaneous temperature to be well reflected at the surface of the skin. The participants remained seated without any body parts in contact with one another and without removing their feet from the carpet to ensure that the surface temperature of their skin correctly reflected the internal temperature of the joints and limbs.

The joints of interest were the same for the control subjects and patients with RA - elbows, wrist and hand joints, knees, ankles and feet joints. Each articulation was captured in different anatomical views.

The images of extended elbows were taken in the posterior and anterior view of the frontal anatomical plane. The elbows flexed at 90 degrees were imaged in the frontal plane. The flexed elbows were also imaged in the right and left median plan. See below grey level TIR images that were taken of the elbows. 
Table 3-1: Grey Level Thermal Infrared Views of the Elbow

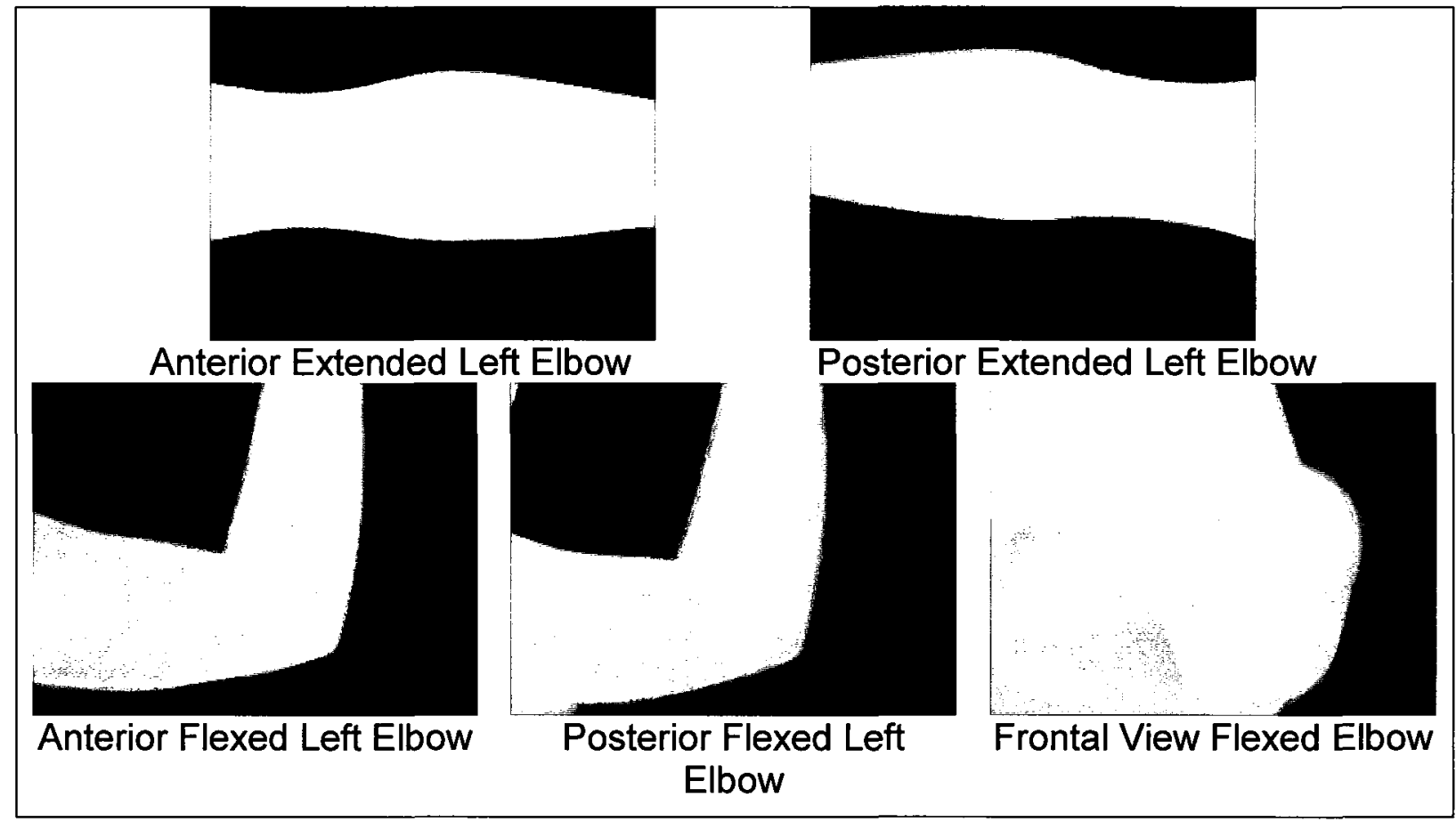

The extended wrists and hand joints were imaged in a single caption in the posterior and anterior view of the frontal anatomical plane. See below grey level TIR images that were taken of the wrist and hand joints.

Table 3-2: Grey Level Thermal Infrared Views of the Wrist and Hand

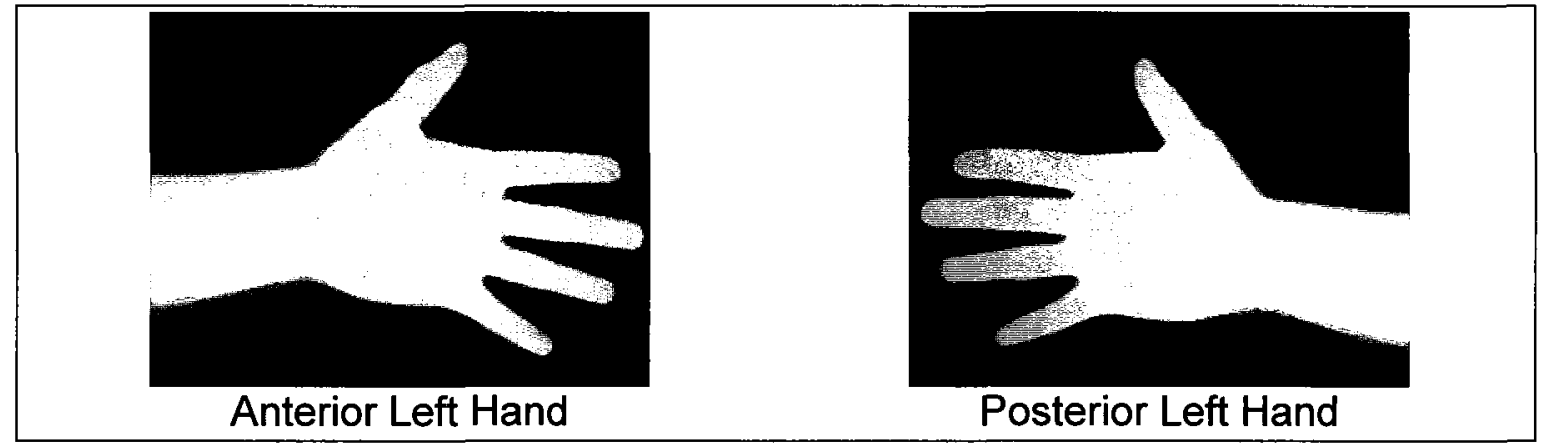

Images of the extended knees were taken in the posterior and anterior view of the frontal anatomical plane. Captions were taken of both extended 
knees at one time and of individual extended knees separately. Images were also captured in the sitting position (knees flexed at 90 degrees) in the anterior frontal view. Captions were taken of both flexed knees at one time and of individual flexed knees separately. The individual flexed knees were also imaged in the right and left median plan. See below grey level TIR images that were taken of the knees.

Table 3-3: Grey Level Thermal Infrared Views of the Knee

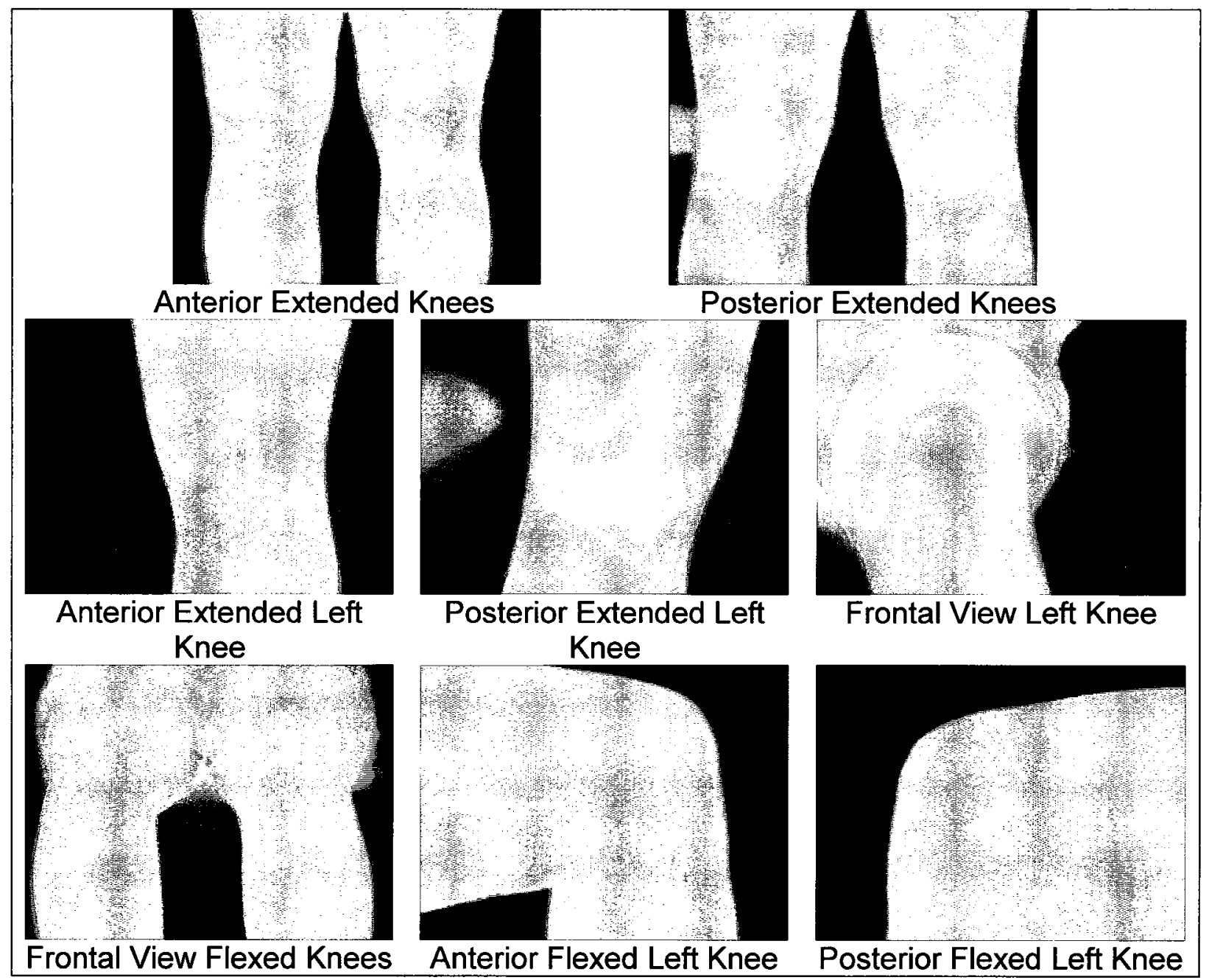


Images of the ankles and feet joints were taken in the posterior view of the frontal anatomical plane. Posterior captions were taken of both ankles at one time and of individual ankles separately. Images were also captured in the right and left median plan of the ankles and feet joints. The top and the bottom of the feet were also imaged. The top of the feet were taken at two distances $-0.5 \mathrm{~m}$ and $1 \mathrm{~m}$ respectively. Top views were taken of both feet at one time and of individual feet separately. The bottoms of the feet were taken at the end of the session in two rounds. The first round was captured immediately after the imaging session and the second round was captured after a 5 minute resting and cooling period. During this cooling period, the feet remained propped up and relaxed on a stool to allow the skin surface of the feet to efficiently represent the intra-articular temperature of all the feet joints. See below grey level TIR images that were taken of the ankles and feet joints.

Table 3-4: Gray scale Level Thermal Infrared Views of the Ankle and Foot

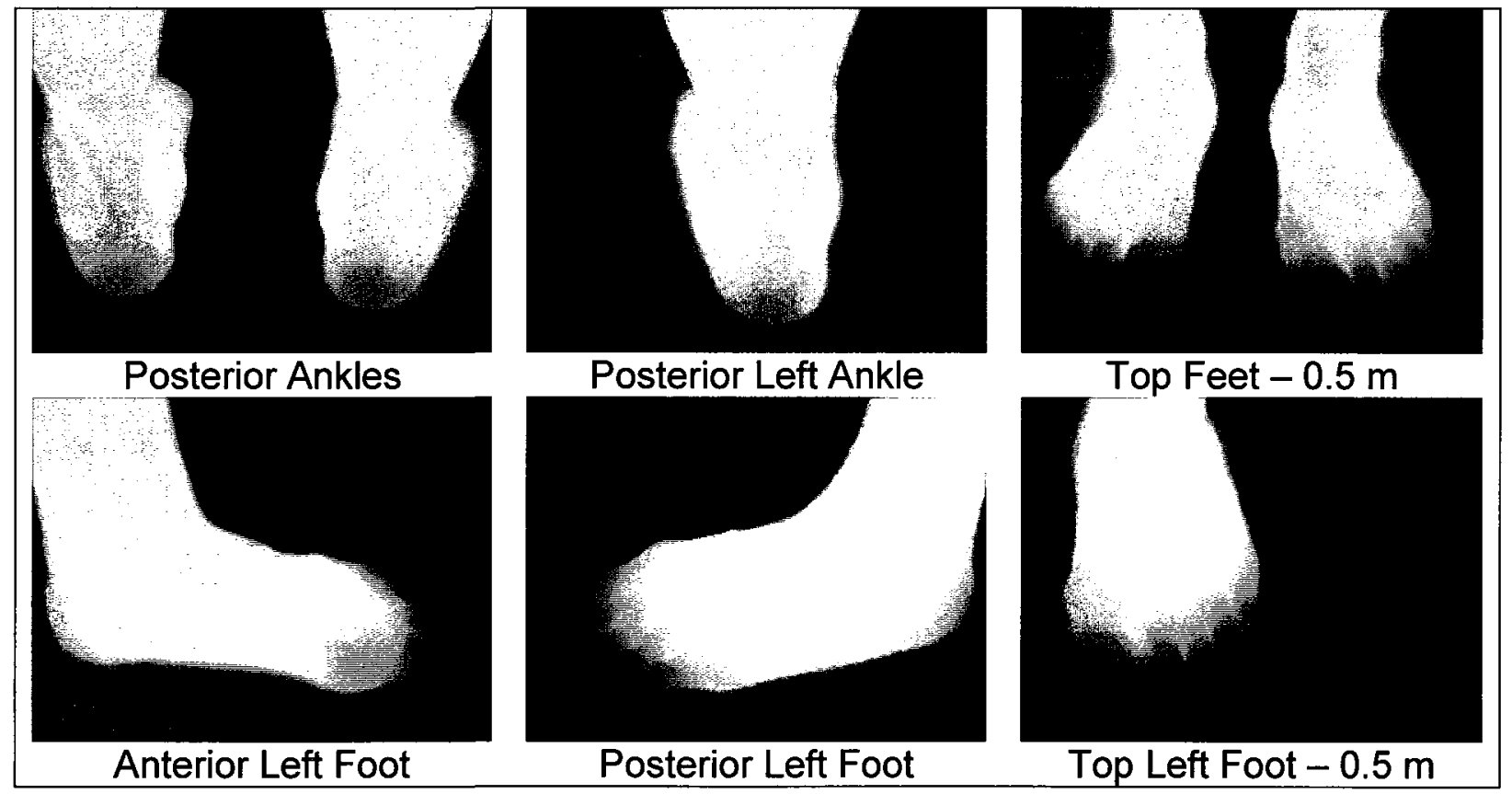




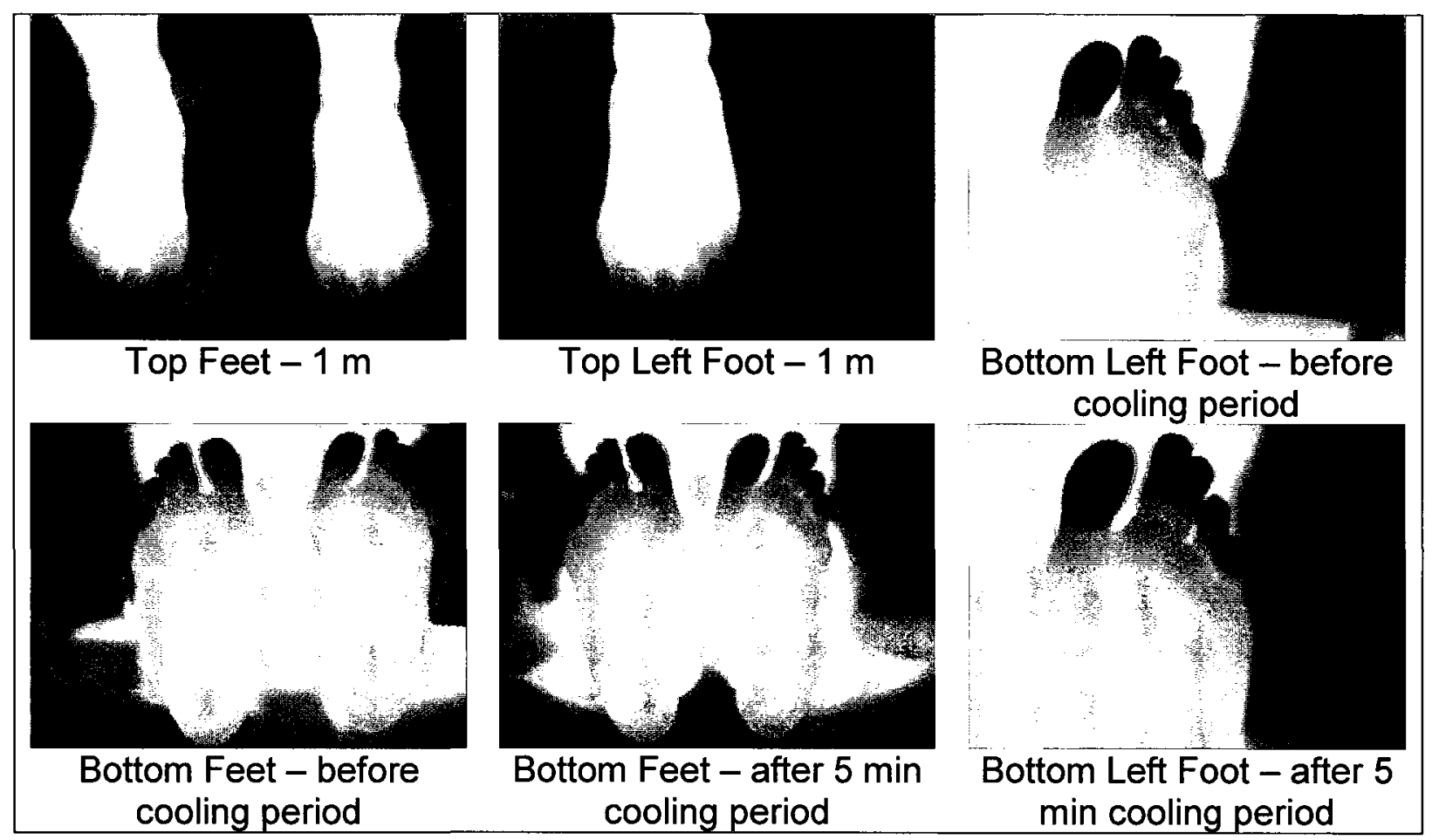

\subsection{Analysis of Data}

To properly analyse the data of the TIR images, it is important to first review the goals of the thesis. The first objective of this thesis is to determine if it is possible to discriminate between the control group and the RA group. The second objective is to identify the best joints to examine in a clinical setting that best determines if a participant has or does not have RA. The third objective of this thesis is to identify the best mathematical calculation of the data to assess the presence or absence of RA. The fourth objective is to determine if thermal IR images can be used to classify the RA patients into three severity groups - high degree of RA, moderate degree of RA and low degree of RA. The final objective of this thesis is to observe the effect of age on the thermal IR measurements. 


\subsubsection{Converting the Thermal IR Images into Data for analysis}

To accomplish the first three goals specifically each *.seq FLIR file is converted into a *.mat MATLAB file using the FLIR software. The image - now a matrix of temperatures in Kelvin - is transformed into a normalized matrix ranging from 0 to 1 using the mat2gray function in MATLAB. When the normalized matrix is displayed via MATLAB's imshow function, a gray scale image is produced. These images are displayed in gray scale to eliminate any biases that a pseudo-colour image may create when the interpreter is viewing the image. It has been shown that the use of pseudo-colours when interpreting TIR images is not a good practice given that humans are subject to psychological effect of hot and cold colours (Anbar 1991, 479-484).

The Regions of Interests (ROIs) are manually selected from the gray scale image using anatomical reference points and the MATLAB's roipoly function. The anatomical reference points are determined by studying the anatomy of each joint to determine the location of the synovial membrane (Moore, 2007). The joints analysed are the $2^{\text {nd }}$ and $3^{\text {rd }}$ MCPs, the $2^{\text {nd }}$ and $3^{\text {rd }}$ PIPs, the wrist and the knee. The main anatomical point that is used to select the region of interest of the knee was the patella. The synovial joint is found underneath the patella. The anatomical points that are used to select the region of interest of the wrist are the pisiform bone - the small pea shaped wrist bone on the inner side (ulnar side) of the forearm - and the base of the thumb - were the trapezium and scaphoid bones are found. Please refer to figure 1.3 for the location of these bones. The anatomical points that are used for the MCP and PIP joints are the 
MCP and PIP articulations themselves. The MCP and PIP joints are identified by their ovoid surface. The synovial joint is found directly under the ovoid surface. The images below demonstrate the gray scale matrix of the original TIR image with the manually selected ROIs.

Table 3-5: Regions of Interests Manually Selected for the $2^{\text {nd }}$ and $3^{\text {rd }}$ MCPS, the $2^{\text {nd }}$ and $3^{\text {rd }}$ PIPs, the Wrist and the Knee

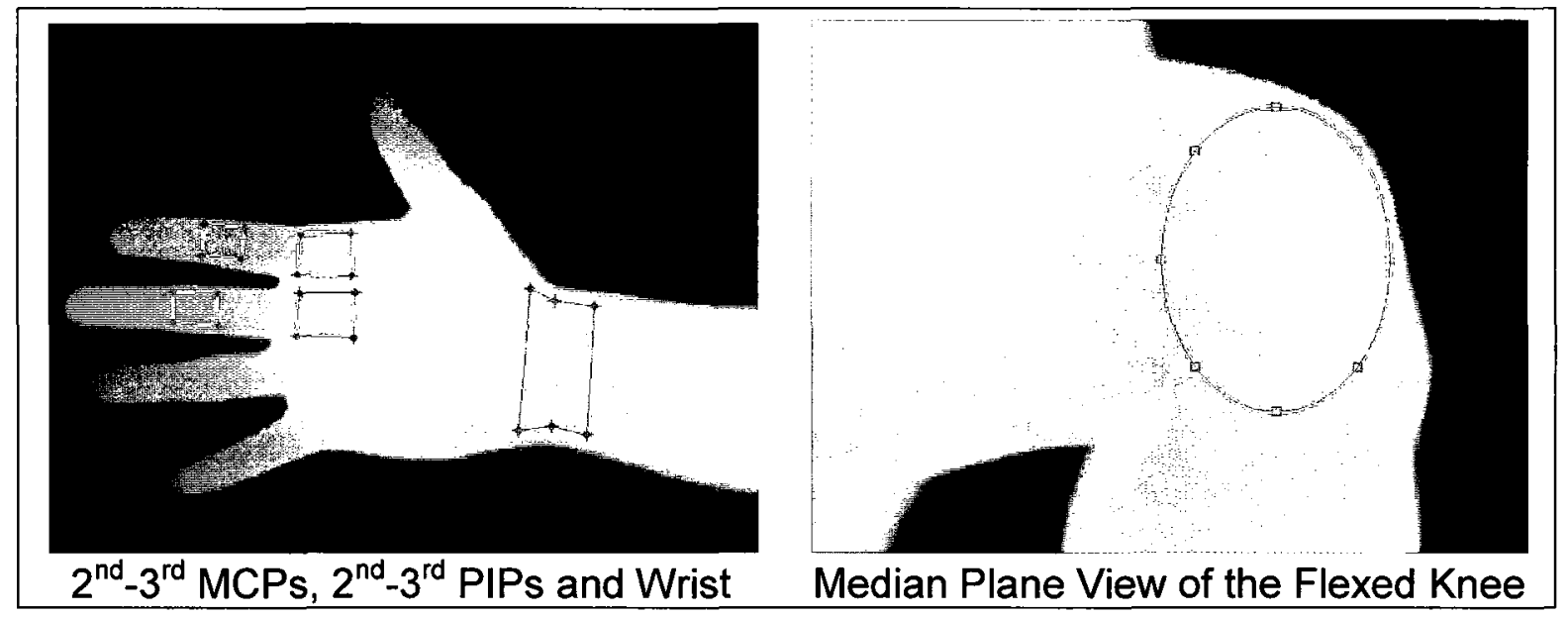

Once the joints are identified, MATLAB outputs an array of temperature values which represent the temperature of every pixel within the selected region of interest of the TIR image. The resulting array is a probability distribution of a real-valued random variable.

Measurements are then extracted from these arrays to be analysed: max, min, mean, median, mode, standard deviation, skewness and kurtosis. The max and min measurements refer to the maximum and minimum temperatures within the array of the joint. The mean is the average temperature value of the elements of the array. If there are $\mathrm{N}$ elements in the array then the mean value is the sum of all element temperatures divided by $\mathrm{N}$. The median is the 
temperature value of the middle element of the joint's array when all the elements of the array are arranged in either ascending or descending order according to their temperature values. If there are $\mathrm{N}$ elements in the array, the median is the value of the $(\mathrm{N}+1) / 2$ element of the ordered values of the array. The mode is the temperature value that occurs most frequently in the array of the joint. The mean, median and mode are measures of central tendency - the location of a distribution. The standard deviation is a measure of dispersion and refers to the variability of the array distribution around the mean value. The skewness and the kurtosis describe the shape of the distribution. The skewness is a measure of the asymmetry of an array's distribution. A symmetric distribution around its mean has zero skewness. In other words, a symmetric array distribution has zero skewness and equal values of mean, median and mode. A distribution is positively skewed if the right tail is longer with mean > median > mode. A distribution is negatively skewed if the left tail is longer with mode $>$ median $>$ mean. (Salvatore, 2002) The figure below illustrates the different types of skewness.

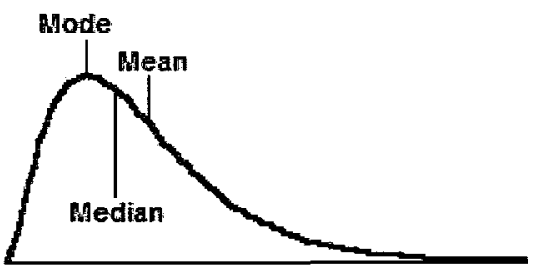

Positively Skewed

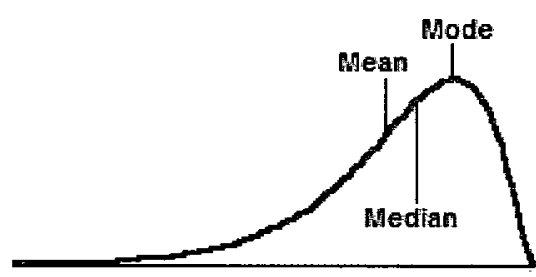

Hegatively Skewed

Figure 3-2: Types of Skewness 
The kurtosis is a description of the peak of the array's distribution. A mesokurtic distribution has zero excess kurtosis and has a normal peak around the mean. An example of a mesokurtic distribution is the normal Gaussian distribution. A distribution that is leptokurtic has a positive excess kurtosis and has a more acute peak around the mean with fatter tails. An example of a leptokurtic distribution is the Laplace distribution. A platykurtic distribution has a negative excess kurtosis and has a lower wider peak around the mean with thinner tails. An example of a leptokurtic distribution is the Bernoulli distribution. (Salvatore, 2002) The figure below illustrates the different types of kurtosis.

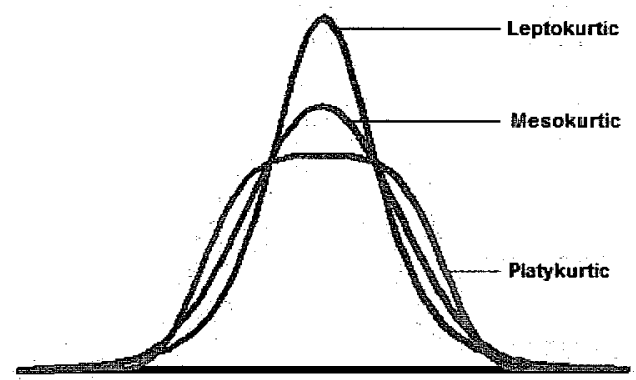

Figure 3-3: Types of Kurtosis

The literature review has shown that certain measurements (such as max, min, mean, median, mode) extracted from the TIR images are subject to interparticipant variation and many other factors (such as time of day, vascular distribution of the patient, environment, etc.). To eliminate the possibility that a person might simply be naturally warmer or colder than another, these measurements are slightly modified. Inspired by the Heat Distribution Index and Salisbury's research, Ratios, differences and dispersion measurements (variance) are used to compare the control samples and the patient samples. Ratios, differences and dispersion measurements are based on the thermal 
distribution of the surface of the skin rather than the exact temperature reading, thus eliminating inter-participant differences. The modified datasets that are used for the analysis were:

Skewness
Kurtosis
Variance
Mode/Max
Median/Max
Min/Max

\author{
Max-Min \\ (Mode-Mean) $^{2}$ \\ Mode/Min \\ Median/Min \\ Mean/Min
}

These modified datasets are referred to as matrices from this point on in the thesis. The matrix is a probability distribution of a real-valued random variable.

Salisbury's HDI is based on the dispersion (the variance) of the distribution of the thermal surface over the joint. In this thesis, many other measures of dispersion are used to characterize different dispersion aspects of the matrix. For instance, the skewness and kurtosis are direct representations of the temperature distribution's shape and thus indirectly represent the dispersion. The variance is the standard deviation (STD) squared and is the typical measurement of dispersion. The (Mode-Mean) ${ }^{2}$ is the mode temperature minus the mean temperature squared. It represents the dispersion range between the mode temperature and the mean temperature.

To further explore matrices that eliminate inter-participant variation, ratios are also explored: Mode/Max, Median/Max, Mode/Min, Median/Min and Mean/Min are calculated by dividing the numerator temperature (either mode, median or mean) by the denominator temperature (either max or min). These 
ratios normalize the exact temperature value with the participant's own maximum or minimum temperature value. These ratios eliminate the error caused by a naturally colder or warmer participant.

The Max-Min matrix is simply the maximum temperature minus the minimum temperature and represents the temperature range of the joint region. This range is explored to see if the matrix can be used to correctly represent the temperature range of control subjects and RA patients.

The hypothesis is that the dispersion, ratio and difference matrices may be a better representation of the presence or absence of RA in participants.

\subsubsection{First Objective - Discriminate Between the Control Group and the Patient Group}

To answer the first objective additional data is extracted from the image before the statistical analysis stage. A MATLAB function is used to identify the pixels within the image that ranged from the mode temperature to the maximum temperature. The hypothesis is that the patients have more pixels within this range than the control group since RA patients are warmer at the surface of the affected joint and have abnormal hot spots present. To calculate the amount of pixels from the mode temperature to the maximum temperature, MATLAB's roicolor function is used to drive every pixel within that range to 1 and every pixel outside of that range to 0 . This produces a binary black and white image that highlights the hot spots and the higher temperature pixels within the TIR image. An example of the binary image is seen below. 
Table 3-6: The Binary Image Output of MATLAB's roicolor Function which Highlights the Pixels that are in the Mode to Maximum Temperature Range

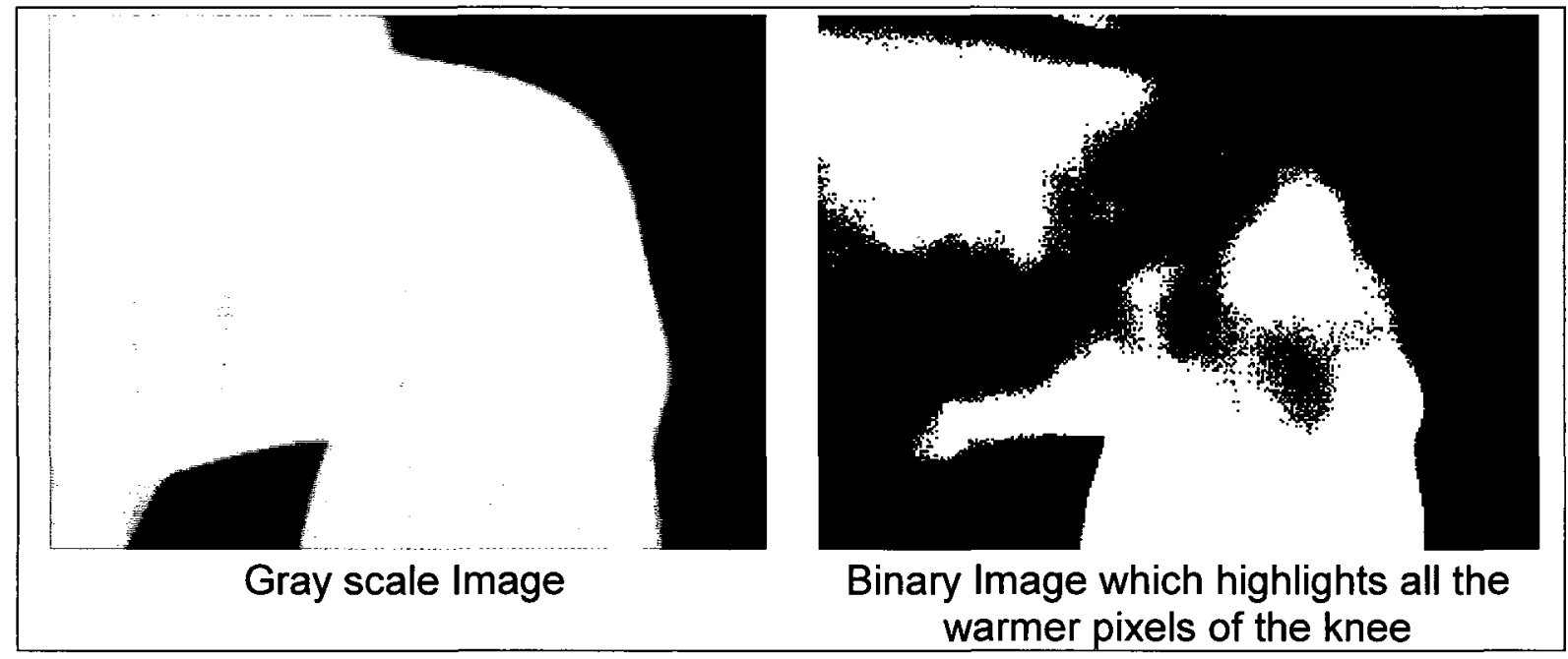

In the binary image, the black pixels are cooler than the white pixels. This type of image allows the user to visually see the temperature distribution of the joint and the presence of hot spots. To mathematically quantify the result, the sum of the pixels highlighted are counted and presented as the percentage of the joint that is covered by warmer pixels and/or hot spots.

The hypothesis of the first objective is that the matrices presented above and the percentage of the joint covered by warmer pixels will allow the user to discriminate between the control group and the patient group.

Once the matrices and percentages are extracted and calculated, statistical tests are applied to the results to satisfy the first objective - that the control group is statistically different from the patient group. The first step of the statistical analysis involves testing the distribution of the matrices for normality. If 
the matrices are not normally distributed, then the use of tests derived from the normally distribution such as the t-test and ANOVA are invalid (Salvatore, 2002). The current analysis uses the Lilliefors Test of normality (Matlab, 2009). It is applied to the matrix distributions to see if they were Gaussian. The Lilliefors Normality Test is explained in detail in the next chapter. The second step of the statistical analysis applying hypothesis tests to the matrices. If the matrices are normally distributed, parametric hypothesis tests such as the Student t-Test and the Anova Test, would be used to prove the statistical significance of the results. If the matrices are not normally distributed, then non-parametric hypothesis tests, such as the Kruskal-Wallis Test (Salvatore, 2002; Matlab, 2009) and the Ranksum Test (Matlab, 2009), would be used to prove the statistical significance of the results. The hypothesis tests used are explained in detail in the next chapter. To satisfy the first objective, these hypothesis tests should show that the control group matrix distributions are statistically different from the patient group matrix distributions at a significant level of at least $5 \%(\alpha=0.05)$ and with a $p$-value lower than 0.05 . The values for $\alpha$ and $p$ are the generally accepted values for statistical significance in hypothesis testing (Salvatore, 2002).

3.4.3 Second Objective - Identify the Joints that Best Determine if a Participant has or does not have RA

To answer the second objective the joints analysed are the $2^{\text {nd }}$ and $3^{\text {rd }}$ MCPs, the $2^{\text {nd }}$ and $3^{\text {rd }}$ PIPs, the wrist and the knee. The hypothesis is that the MCPs and the knees are the best joints in a clinical setting to best determine if a 
participant has or does not have RA. Verbal discussions with Dr. Jacob Karsh confirmed that in the majority of RA cases the MCPs are the joints that are most affected early on in the disease - (Dr. Jacob Karsh, Personal Communication, September $22^{\text {nd }} 2009$ ). Furthermore, the knees are expected to show good results since past research has shown that the knees are the best joint to observe the presence of RA. The knee is also a larger joint compared to the others resulting in a larger matrix with more elements (since there are more pixels in the TIR image over this joint); therefore the knee matrices should show better statistical results of the thermal distribution.

The MCPs, PIPs and wrists are analysed using the posterior view of the hand in the frontal plane, since the skin surface on the back of the hand is a better representation of the intra-articular temperature. The reason the back of the hand is a better representation is because the skin surface of the palms are subject to more heat transfer when the participant moves around and accidentally touches other body parts or objects. The knees are analysed using the anterior view of the flexed knees in the median plane. The views of the extended and flexed knees in the frontal plane is not expected to be a good representation of the intra-articular temperature since the patella acts as a thick barrier between the joint's temperature and the skin surface temperature. The anatomical views that were used for the hands and knees are shown in the table below. 
Table 3-7: Anatomical Views of the Hand and Knee Used for Analysis

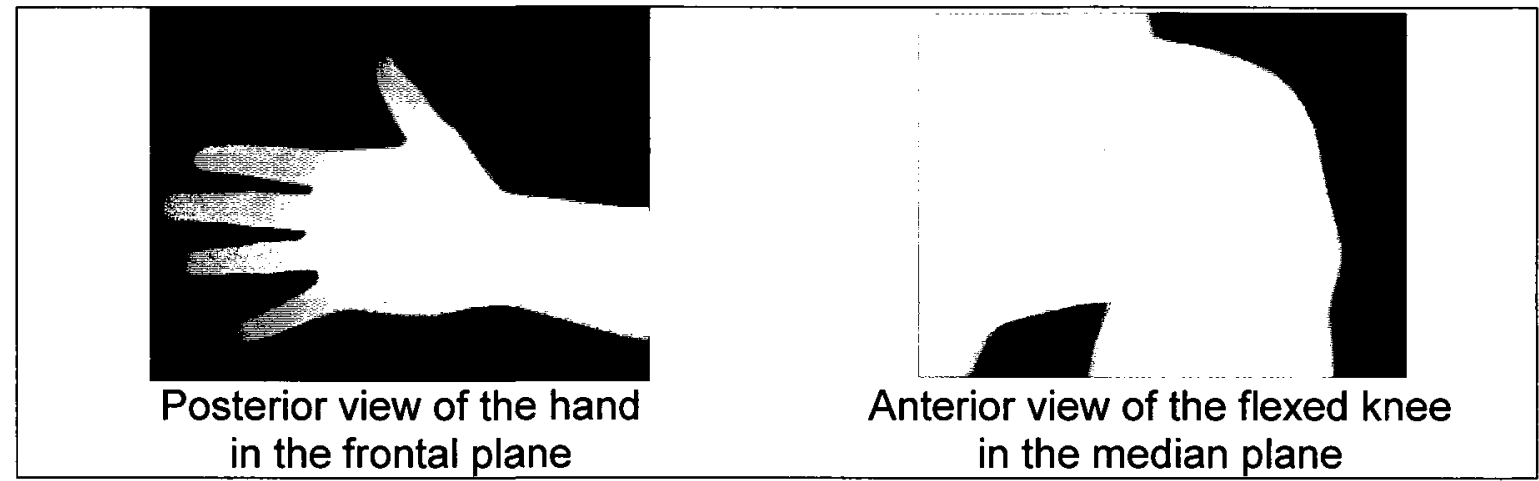

The measurements are extracted using the same methods described above and the ratio/difference/dispersion matrices are calculated for statistical analysis. The same statistical tests are applied to the matrices as explained in objective one to prove the statistical significance of the best joint. First, the Lilliefors Normality test is applied to observe if the matrix distributions are Gaussian. Second, the correct hypothesis tests (parametric or non-parametric) are used to prove which joints show the most distribution difference between the control and patient group. To satisfy the second objective, these hypothesis should be tested with a significant level of at least $5 \%(\alpha=0.05)$ and with a $p$ value lower than 0.05 . The values for $\alpha$ and $p$ are the generally accepted values for statistical significance in hypothesis testing (Salvatore, 2002).

3.4.4 Third Objective - Identify the Matrix that Best Assesses the Presence or Absence of RA

To answer the third objective it is important to restate the matrices that are used for analysis: 
Skewness

Kurtosis

Variance

Mode/Max

Median/Max

Min/Max
Max-Min

(Mode-Mean) $^{2}$

Mode/Min

Median/Min

Mean/Min

The matrices are expected to show a difference between the control group and the patient group. The hypothesis is that the variance, the max-min, the (mode-mean) ${ }^{2}$ will best represent the thermal distribution dispersion to statistically distinguish between the control and patient group since they are dispersion measurements. The control group is expected to have a larger range of temperatures (a higher variance, a higher max-min and a higher (modemean $)^{2}$ ) since they are expected to have a distribution that ranges from colder temperatures to the normal temperatures of the joint. The patient group is expected to have a lower variance, a lower max-min and a lower (mode-mean) ${ }^{2}$ since their joints have fewer cold regions and their temperature range will begin at a warmer temperature than the control subjects, thus making the temperature range over the joints smaller than the control subjects.

The values in the matrix go through the same normality and hypothesis testing as explained in objectives one and two. To satisfy the third objective, these hypothesis tests should emphasize the matrices that show the largest statistical difference between the best joints of the control group and the best joints of the patient group at a significant level of at least $5 \%(\alpha=0.05)$ and with a $p$-value lower than 0.05 . The values for $\alpha$ and $p$ are the generally accepted values for statistical significance in hypothesis testing (Salvatore, 2002). 


\subsubsection{Fourth Objective - Classify RA Patients into Three Severity Groups}

To study the fourth objective, the best matrices chosen from objective three are extracted from the TIR images of the patient group to represent the thermal distribution of the best joints found in objective two. Dr. Jacob Karsh identified each of the 13 patients as having "high", "moderate" or "low" degree of RA. The 13 patients are therefore ranked by severity according to Dr. Karsh's clinical assessment - (Dr. Jacob Karsh, Personal Communication, July $23^{\text {rd }}$ 2009). Then, the elements of the best matrix of the best representing joint are ranked according to their element values. As a result, the patients are ranked by RA severity done by the rheumatologist and ranked by element values within the matrix extracted from the measurements of the TIR image. These two rankings are compared using the Spearman Correlation Coefficient (Salvatore, 2002) to see whether the matrix distribution can correctly rank the patients by severity. The Spearman Correlation Coefficient is explained in detail in the next chapter. To satisfy the fourth objective, the Spearman Correlation Coefficient should show that the matrix ranking of the measurements highly correlates with the severity rankings done by the physician. A high correlation is a Spearman Coefficient greater than 0.5 .

\subsubsection{Fifth Objective - Observe the Effect of Age on Thermal IR Measurements}

To study the fifth objective, the best matrices (from objective three) of the best representative joints (from objective two) are analysed from the control group. The 18 control subjects are ranked by age group: 10-20 years of age, 20- 
30 years of age, $30-40$ years of age, $40-50$ years of age, $50-60$ years of age and 60-70 years of age. Then, the elements of the best matrix of the best representing joints are ranked according to their element values. The control subjects are also ranked by age group and ranked by their matrix values. These two rankings are compared using the Spearman Correlation Coefficient to see whether the matrix distribution is highly correlated to the matrix elements. To satisfy the fifth objective, the Spearman Correlation Coefficient should show the extent to which age correlates with the TIR results represented in the matrix distribution. If the Spearman Correlation Coefficient is greater than 0.5 , it is possible to state that age is associated strongly to the calculated matrix values derived from the TIR images. If the Spearman Coefficient is lower than 0.5 , it is possible to state that age is associated weakly to the calculated matrices.

The following chapter presents the results and analysis of the measurements and matrices extracted from the TIR images. 


\section{CHAPTER 4}

\section{RESULTS AND ANALYSIS}

Chapter 4 begins by displaying the resulting matrices derived from the raw data measurements extracted from the thermal infrared images. A discussion of the Lilliefors Normality Test, which determines whether the matrix distributions are Gaussian, follows. In addition, hypothesis tests are done to show the statistical significance of the results to see if each thesis objective can be met.

\subsection{Results}

All raw data measurements that are extracted from the original Thermal InfraRed (TIR) images can be found in Appendix D. The matrices used for analysis in this chapter are all calculated from the raw measurements.

These raw data measurements were presented at the IEEE International Workshop on Medical Measurements and Applications (MeMeA) in Cetraro, Italy in May 2009. The paper presented entitled "Preliminary Results of Severity of Illness Measures of Rheumatoid Arthritis Using Infrared Imaging" can be found in Appendix F.

The table below shows the average value for each matrix of every joint for the control and patient groups. 
Table 4-1: Average Values for Each Matrix of Every Joint for the Control and Patient Group

\begin{tabular}{|c|c|c|c|c|c|c|c|c|}
\hline & \multicolumn{7}{|c|}{ Average Value for: } \\
\hline & & Variance & \multicolumn{2}{|c|}{ Mode/Max } & \multicolumn{2}{|c|}{ Median/Max } & Min/Max & Max-Min \\
\hline \multirow{2}{*}{ 2nd MCP } & Control & 0,13997 & \multicolumn{2}{|c|}{0,99645} & \multicolumn{2}{|c|}{0,99680} & 0,99430 & 1,72480 \\
\hline & Patient & 0,14211 & \multicolumn{2}{|c|}{0,99725} & \multicolumn{2}{|c|}{0,99712} & 0,99378 & 1,89925 \\
\hline \multirow[t]{2}{*}{ 3rd MCP } & Control & 0,08825 & \multicolumn{2}{|c|}{0,99114} & \multicolumn{2}{|c|}{0,99738} & 0,99552 & 1,35634 \\
\hline & Patient & 0,06282 & \multicolumn{2}{|c|}{0,99328} & \multicolumn{2}{|c|}{0,99781} & 0,99621 & 1,15770 \\
\hline \multirow[t]{2}{*}{ 2nd PIP } & Control & 0,07617 & \multicolumn{2}{|c|}{0,99771} & \multicolumn{2}{|c|}{0,99793} & 0,99584 & 1,25547 \\
\hline & Patient & 0,07074 & \multicolumn{2}{|c|}{0,99776} & \multicolumn{2}{|c|}{0,99798} & 0,99554 & 1,36027 \\
\hline \multirow[t]{2}{*}{ 3rd PIP } & Control & 0,07945 & \multicolumn{2}{|c|}{0,99817} & \multicolumn{2}{|c|}{0,99828} & 0,99562 & 1,32301 \\
\hline & Patient & 0,07504 & \multicolumn{2}{|c|}{0,99826} & \multicolumn{2}{|c|}{0,99817} & 0,99517 & 1,47468 \\
\hline \multirow{2}{*}{ Wrist } & Control & 0,13762 & \multicolumn{2}{|c|}{0,99736} & \multicolumn{2}{|c|}{0,99733} & 0,99406 & 1,80680 \\
\hline & Patient & 0,16193 & \multicolumn{2}{|c|}{0,99713} & \multicolumn{2}{|c|}{0,99692} & 0,99337 & 2,02897 \\
\hline \multirow{2}{*}{ Knee } & Control & 0,35935 & \multicolumn{2}{|c|}{0,98889} & 0,99 & & 0,98962 & 3,15889 \\
\hline & Patient & 0,46501 & 0,98 & & 0,996 & & 0,99113 & 2,69858 \\
\hline & & & & & erage $\mathrm{V}$ & UE & & \\
\hline & & (Mode-M & $a n)^{2}$ & $\mathrm{Mc}$ & de/Min & $\mathrm{Me}$ & lian/Min & Mean/Min \\
\hline 2nd MCP & Control & 0,049 & & & 00217 & & 00252 & 1,00256 \\
\hline & Patient & 0,043 & & & 00350 & & 00337 & 1,00334 \\
\hline 3rd MCP & Control & 0,044 & & & 00152 & & 00186 & 1,00193 \\
\hline & Patient & 0,028 & & & 00155 & & 00161 & 1,00167 \\
\hline 2nd PIP & Control & 0,049 & & & 00187 & & 00209 & 1,00209 \\
\hline & Patient & 0,152 & & & 00223 & & 00246 & 1,00248 \\
\hline 3rd PIP & Control & 0,132 & & & 00256 & & 00267 & 1,00260 \\
\hline & Patient & 0,020 & & & 00312 & & 00303 & 1,00295 \\
\hline Wrist & Control & 0,053 & & & 00331 & & 00329 & 1,00319 \\
\hline & Patient & 0,050 & & & 00379 & & 00358 & 1,00354 \\
\hline Knee & Control & 0,129 & & & 00674 & & 00624 & 1,00602 \\
\hline & Patient & 0,269 & & & 00536 & & 00538 & 1,00513 \\
\hline
\end{tabular}

Please note that the control group's averages are calculated from 36 matrix values (18 control subjects $\times 2$ ), since each control subject presented two TIR images per joint - the left joint and the right joint. The patient group's averages are calculated from 26 matrix values (13 RA patients $\times 2$ ). RA is an auto-immune disease affecting the joints bilaterally. As explained in great detail in the introduction chapter, the majority of RA patients show diseased joints on both sides of their body. For instance if the left knee is affected by RA, the patient's 
right knee will be equally affected. Therefore, it is justified to include both the left and the right joints of a particular observed region of interest for the matrix distribution. Using both the right and left TIR images increases the sample size of the matrices; thus making the statistical analysis stronger and the matrix distributions more representative of each group.

\subsection{Analysis and Discussion}

Note that all statistical explanations and equations are derived from MathWork's MATLAB Help files and from D. Salvatore and D. Reagle's "Statistics and Econometrics" book from the Schaum's Outline Series of McGraw-Hill Companies (2002).

\subsubsection{First Objective - Discriminate Between the Control Group and the Patient Group}

First, normality is assessed using the Lilliefors Test for all the matrices calculated from the TIR measurements. Lilliefors tests the null hypothesis that the matrix samples come from a normal distribution against the alternative that they do not come from a distribution in the normal family. It is a 2-sided goodness-of-fit test used when the parameters of the distribution are unknown and must be estimated. The Lilliefors test statistic is:

$$
K S=\max _{x}|S C D F(x)-C D F(x)|
$$

where SCDF is the cumulative distribution function taken from the matrix samples and CDF is the normal cumulative distribution function with the same 
mean and standard deviation as the sample matrix (Matlab, 2009). MATLAB's lillietest uses a table of critical values computed using Monte Carlo simulation for sample sizes less than 1000 and significance levels between 0.001 and 0.50 . The significance level (also known as $\alpha$ or the type-l error) is used to determine the rejection region of the statistical test. Based on $\alpha$ and the degrees of freedom, a critical value is determined and compared to the Lilliefors test statistic stated in the equation above. If Lilliefors test statistic $>$ critical value, then the null hypothesis is rejected. If Lilliefors test statistic < critical value, then the null hypothesis cannot be rejected (Salvatore, 2002). MATLAB outputs four results: $h$ value, $p$ value, $K S$ value and critical value. The $h$ value is 1 if the null hypothesis is rejected and 0 if it cannot be rejected at the $5 \%$ significance level. The significance level is always set to 0.05 in this thesis. The $p$ value is the probability of observing an element from the matrix distribution if the null hypothesis is true. The $K S$ value is the Lilliefors test statistic retrieved from the equation stated above. The critical value is the value that determines the rejection region of the test based on $\alpha$ and the degrees of freedom.

It is important to note that $a$ is a number selected independently of the $p$ value and of the matrix. It is possible to reject the null hypothesis at a statistical significance of $\alpha$ when the $p$-value is smaller than $\alpha$; otherwise it is not possible to reject the null hypothesis at level $\alpha$ (Salvatore, 2002).

Random matrices for random joints were selected to see if any of them were normally distributed using MATLAB's lilliefors function. The table below shows MATLAB's four outputs obtained by the normality test. A reminder that 
the null hypothesis of the test is that the matrix distribution belongs to the normal distribution family; therefore, if $\mathrm{h}$ is 1 with a $\mathrm{p}$-value lower than $\alpha$ (where $\alpha=$ 0.05), it is possible to conclude that the matrix distribution is not a normal distribution.

Table 4-2: MATLAB Lilliefors Test Results for Randomly Selected Matrices

\begin{tabular}{|c|c|c|c|c|c|c|}
\hline \multirow[b]{2}{*}{$\begin{array}{l}\text { Participant } \\
\text { Type }\end{array}$} & \multirow[b]{2}{*}{ Joint } & \multirow[b]{2}{*}{ Matrix Distribution } & \multicolumn{4}{|c|}{ MATLAB Outputs } \\
\hline & & & $\begin{array}{c}\mathbf{h} \\
\text { value }\end{array}$ & $\begin{array}{c}p \\
\text { value }\end{array}$ & $\begin{array}{c}\text { KS } \\
\text { value }\end{array}$ & $\begin{array}{l}\text { Critical } \\
\text { Value }\end{array}$ \\
\hline \multirow{4}{*}{$\begin{array}{l}\text { Control } \\
\text { Subjects }\end{array}$} & 2nd & Skewness & 1 & 0.0482 & 0.1503 & 0.1497 \\
\hline & 3 rd MCP & Kurtosis & 1 & 0.0020 & 0.1955 & 0.1497 \\
\hline & 2nd PIP & Variance & 1 & 0.0026 & 0.1920 & 0.1497 \\
\hline & 3 rd PIP & Minimum Temperature & 1 & 0.0040 & 0.1865 & 0.1497 \\
\hline \multirow[t]{4}{*}{ Patients } & 2nd & Kurtosis & 1 & 0.001 & 0.4422 & 0.1699 \\
\hline & 3rd MCP & Kurtosis & 1 & 0.0346 & 0.1767 & 0.1699 \\
\hline & 2nd PIP & Maximum Temperature & 1 & 0.0019 & 0.2226 & 0.1699 \\
\hline & 3rd PIP & Minimum Temperature & 1 & 0.0133 & 0.1932 & 0.1699 \\
\hline
\end{tabular}

The results from Lilliefors show that the eight matrices representing the thermal distribution of the joints are not normally distributed (since all the $h$ values are 1$)$ at a $5 \%$ significance level $(\alpha=0.05)$. The $K S$ values are all superior to the critical values and the $p$-values are all lower than $\alpha$; therefore it can be confirmed that the matrices are not from the normal Gaussian family.

From the results of the eight randomly selected matrices that were tested for normality, it will be assumed in this thesis that all the matrices are not normality distributed. As a consequence, parametric hypothesis tests cannot be used for the statistical analysis. Instead of the Student t-Test and the Anova Test, the Kruskal-Wallis Test and the Ranksum Test are applied to the matrices.

Second, the Kruskal-Wallis Test and the Ranksum Test are used to show that the control group's matrix distributions are statistically different from the 
patient's matrix distributions, showing that TIR images can be used to discriminate between the control and patient groups. The Kruskal-Wallis Test is the non-parametric version of the classical one-way Anova test and is based on the standard Anova table using ranks rather than the numeric values of individual sample points. The test compares the medians of two or more matrices and returns the $p$-value for the null hypothesis that all samples are drawn from the same distribution (Salvatore, 2002; Matlab, 2009). MATLAB's ranksum test, also known as the Mann-Whitney U-test, is similar to the Kruskal-Wallis test. It is a two sided non-parametric test with the null hypothesis that two matrices are independent samples from identical continuous distributions with equal medians, against the alternative that they do not have equal medians. If the null hypothesis of the Kruskal-Wallis and the Ranksum tests are rejected at a significance level of $5 \%$ with a p-value lower than 0.05 , it is fair to say that the two compared matrices are not from the same distribution and are statistically different (Matlab, 2009).

Every matrix from the control group is compared to every matrix from the patient group for every joint using MATLAB'S ranksum test. The ransksum results are then confirmed via the Kruskal-Wallis test. MATLAB's ranksum test outputs two results: $h$ value, $p$ value. The $h$ value is 1 if the null hypothesis is rejected and 0 if it cannot be rejected at the $5 \%$ significance level. The $p$ value is the probability of observing an element from the matrix distribution if the null hypothesis is true. If the $h$ value is 1 , with a $p$-value lower than 0.05 , it is possible to conclude that the matrices of the control group are statistically 
different from the matrices of the patient group (Matlab, 2009). The table below demonstrates the two MATLAB outputs from the ranksum test.

Table 4-3: MATLAB's Ranksum Test Results Comparing the Matrix Distributions of the Control and the Patient Group

\begin{tabular}{|c|c|c|c|c|c|c|c|}
\hline Joint & Matrix & $\begin{array}{c}\text { p- } \\
\text { value }\end{array}$ & $\begin{array}{c}\mathbf{h} \\
\text { value }\end{array}$ & Joint & Matrix & $\begin{array}{c}\text { p- } \\
\text { value }\end{array}$ & $\begin{array}{c}\mathbf{h} \\
\text { value }\end{array}$ \\
\hline \multirow[t]{11}{*}{ MCP2 } & $\begin{array}{l}\text { STD } \\
\text { Skewness }\end{array}$ & $\begin{array}{l}0.3828 \\
0.0136\end{array}$ & $\begin{array}{l}0 \\
1\end{array}$ & \multirow[t]{11}{*}{ PIP2 } & $\begin{array}{l}\text { STD } \\
\text { Skewness }\end{array}$ & $\begin{array}{l}0,9346 \\
01417\end{array}$ & $\begin{array}{l}0 \\
0\end{array}$ \\
\hline & Kurtosis & 0,9346 & 0 & & Kurtosis & 0,0962 & 0 \\
\hline & Mode/Max & 0,0105 & 1 & & Mode/Max & 0,2476 & 0 \\
\hline & Median/Max & 0,0654 & 0 & & Median/Max & 0,2356 & 0 \\
\hline & Min/Max & 0,7484 & 0 & & Min/Max & 0,6599 & 0 \\
\hline & Variance & 0,3828 & 0 & & Variance & 0,9346 & 0 \\
\hline & Max-Min & 0,8171 & 0 & & Max-Min & 0,5912 & 0 \\
\hline & (Mode-Mean) $^{2}$ & 0,8521 & 0 & & (Mode-Mean) $^{2}$ & 0,2298 & 0 \\
\hline & Mode/Min & 0,0173 & 1 & & Mode/Min & 0,2021 & 0 \\
\hline & Median/Min & 0,2861 & 0 & & Median/Min & 0,2356 & 0 \\
\hline & Mean/Min & 0,4603 & 0 & & Mean/Min & 0,2241 & 0 \\
\hline \multirow[t]{12}{*}{ MCP3 } & STD & 0,0153 & 1 & \multirow[t]{12}{*}{ PIP3 } & STD & 0,7484 & 0 \\
\hline & Skewness & 0,4786 & 0 & & Skewness & 0,3360 & 0 \\
\hline & Kurtosis & 0,1969 & 0 & & Kurtosis & 0,0011 & 1 \\
\hline & Mode/Max & 0,0212 & 1 & & Mode/Max & 0,3993 & 0 \\
\hline & Median/Max & 0,0361 & 1 & & Median/Max & 0,3285 & 0 \\
\hline & Min/Max & 0,0047 & 1 & & Min/Max & 0,4694 & 0 \\
\hline & Variance & 0,0153 & 1 & & Variance & 0,7484 & 0 \\
\hline & Max-Min & 0,0074 & 1 & & Max-Min & 0,4162 & 0 \\
\hline & (Mode-Mean) $^{2}$ & 0,011 & 1 & & (Mode-Mean) $^{2}$ & 0,0612 & 0 \\
\hline & Mode/Min & 0,4786 & 0 & & Mode/Min & 0,4603 & 0 \\
\hline & Median/Min & 0,1055 & 0 & & Median/Min & 0,7371 & 0 \\
\hline & Mean/Min & 0,0289 & 1 & & Mean/Min & 0,7371 & 0 \\
\hline \multirow[t]{12}{*}{ Wrist } & STD & 0,3747 & 0 & \multirow[t]{12}{*}{ Knee } & STD & 0.0341 & 1 \\
\hline & Skewness & 0,4248 & 0 & & Skewness & 0,1585 & 0 \\
\hline & Kurtosis & 0,8404 & 0 & & Kurtosis & 0,2508 & 0 \\
\hline & Mode/Max & 0,3993 & 0 & & Mode/Max & 0,2226 & 0 \\
\hline & Median/Max & 0,0796 & 0 & & Median/Max & 0,0318 & 0 \\
\hline & Min/Max & 0,1226 & 0 & & Min/Max & 0,0027 & 1 \\
\hline & Variance & 0,3747 & 0 & & Variance & 0,0341 & 1 \\
\hline & Max-Min & 0,1121 & 0 & & Max-Min & 0,0026 & 1 \\
\hline & (Mode-Mean) ${ }^{2}$ & 0,8697 & 0 & & (Mode-Mean) ${ }^{2}$ & 0,6635 & 0 \\
\hline & Mode/Min & 0,2129 & 0 & & Mode/Min & 0,0072 & 1 \\
\hline & Median/Min & 0,4973 & 0 & & Median/Min & 0,0069 & 1 \\
\hline & Mean/Min & 0,4077 & 0 & & Mean/Min & 0.0128 & 1 \\
\hline
\end{tabular}

*MCP2 represents the $2^{\text {nd }} \mathrm{MCP}, \mathrm{MCP} 3$ represents the $3^{\text {rd }} \mathrm{MCP}$ and thusly for the PIP joints. 
Note that the Kruskal-Wallis results yield the same results as the ranksum test, thereby verifying the hypothesis test results above.

The table above highlights in gray the matrices where the null hypothesis is rejected at a significance level of $5 \%$ with a p-value that was lower than a. These highlighted test results are the ones that were successful in discriminating between the control group and the RA group matrices. Objective one is therefore satisfied since:

1- the skewness, mode/max, mode/min matrices of the $2^{\text {nd }} M C P$ are statistically different distributions for the control and patient group;

2- The standard deviation (STD), mode/max, median/max, $\min / \mathrm{max}$, variance, max-min, (Mode-Mean) ${ }^{2}$, Mean/Min matrices of the $3^{\text {rd }}$ MCP show a statistical difference between the control and patient distributions;

3- The control group's kurtosis matrix of the $3^{\text {rd }}$ PIP is statistically different than the patient's kurtosis matrix of the $3^{\text {rd }} \mathrm{PIP}$;

4- The standard deviation, $\min / \max$, variance, $\max -\min , \operatorname{mode} / \mathrm{min}$, median/min, mean/min matrices of the knee are statistically different distribution for the control and patient group.

To graphically see the difference between the control and patient matrices, histograms are created for a selected matrices mentioned above. These histograms are included in Appendix $E$, but an example is shown below. The histograms clearly show that the matrix of the patients is shifted differently compared to the matrix of the control subjects. The bin containing the most 
elements for the control group is never the same as the bin containing the most elements for the patient group. See example below.

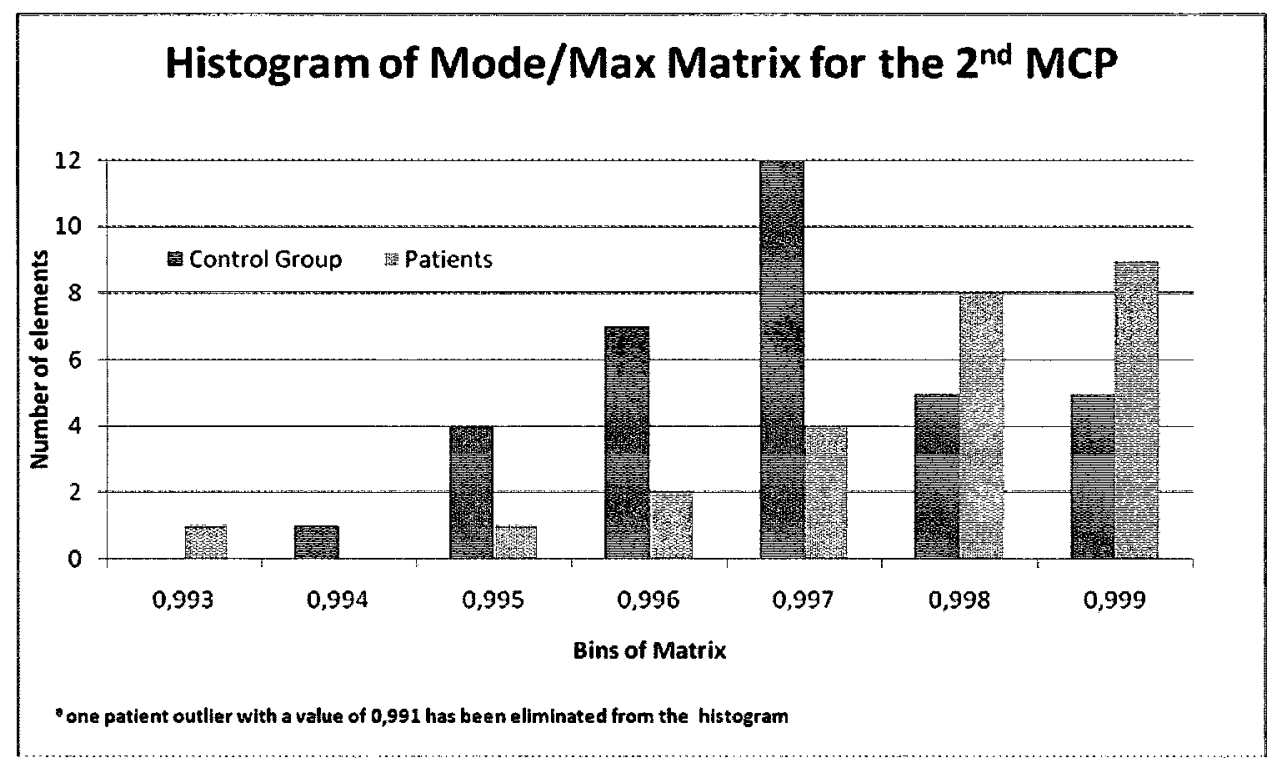

Figure 4-1: Histogram of the Mode/Max Matrix for the 2nd MCP

The histogram shows that the control subjects' $2^{\text {nd }}$ MCP mode/max matrix is shifted to the left whereas the patient's mode/max matrix is more shifted to the right. The bin containing the most elements in the control's matrix is the 0.997 bin whereas the bin containing the most elements in the patient's matrix is 0.999 . It is clear that the distributions of the mode/max matrix can distinguish between the control and patient groups.

To further satisfy objective one, the binary images that are produced using MATLAB's roicolor function are explored. This function highlights (drives to 1 ) all the pixels of the TIR image that are within the temperature range from mode to max; and drives to 0 all the pixels outside of that range. The tables below show 
the resulting images that are produced by MATLAB'S roicolor function for some control subjects and some patients.

Within the control group it is obvious from the binary images below that the knee gradient, as previously discussed in the literature review, are positive from the patella to the inside of the flexed knee. In other words, the patella region has a low thermal temperature and this temperature increases as you get closer to the inside of the flexed knee where the temperature is the warmest. Moreover, there are no evident hot regions within the ROI. It is therefore possible to show via these binary images that control subjects could be identified with a uniform positive temperature gradient and with the absence of evidently abnormal hot regions.

Table 4-4: Grey Images and their Corresponding Binary Images of Control Subjects

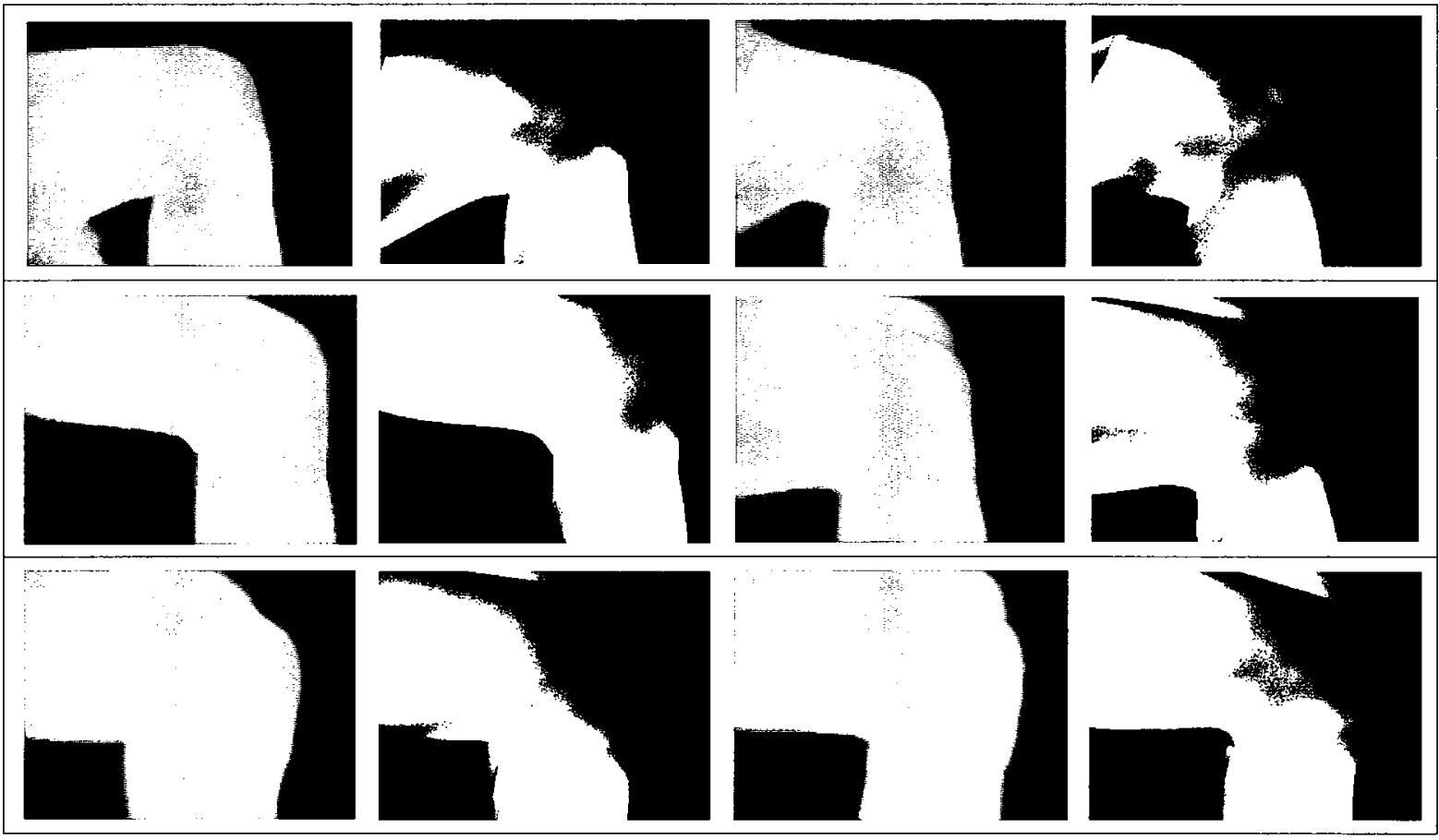


From the binary RA patient images below, it is clear that the patient's temperature distribution gradient from the patella to the inside of the flexed knee is not always uniform or positive. In many cases the temperature gradient has spikes and is negative (going from coolest to warmest). Moreover, there are obvious hot spots throughout the joint. It is possible to show via these binary images that RA patients could be identified with a non-uniform temperature gradient and with the presence of abnormal hot regions.

Table 4-5: Grey Images and their Corresponding Binary Images of Patients

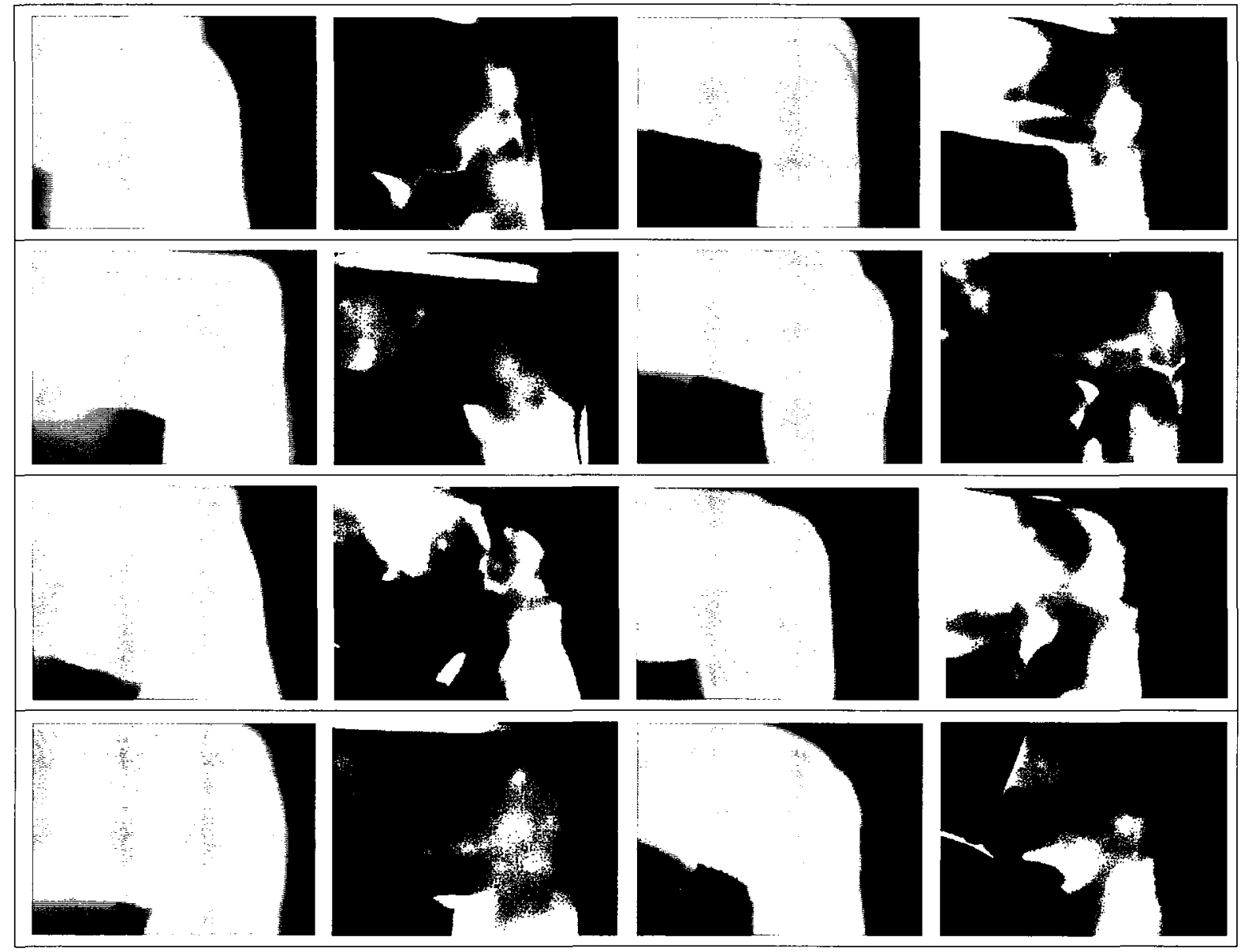


The non-uniform and negative thermal gradients seen in the RA patient's images can be explained by the increase in vascularity caused by synovitis and the proliferation of the synovial cells - the pannus. Blood is also shunted from the deep circulation to the subcutaneous circulation also adding to the increase in temperature at the surface of the joint (Nielsson 1980, 516-528). In RA, there are also chemical mediators and an increase in chemical activity within the intraarticular joint due to the inflammation process as explained in chapter 1 . All of these pathophysiological aspects can explain the abnormalities seen in the patient TIR images.

Since a visual difference is clear between the control and patient groups, statistical hypothesis tests are applied. The percentage of the pixels highlighted (driven to 1) within the joint surface area was calculated for each patient and control. This percentage represents the area of the joint that contained warmer pixels and/or hot spots. The tables below show the percentages extracted from these binary images for both groups. 
Table 4-6: Percentage of Pixels Within the Range Mode to Maximum Temperature Representing the Area of the Joint that is Covered with Warmer Pixels and/or Hot Regions

\begin{tabular}{|c|c|c|c|c|}
\hline & $\begin{array}{c}\text { Participant } \\
\#\end{array}$ & $\begin{array}{c}\text { Number of } \\
\text { Pixels } \\
\text { within the ROI }\end{array}$ & $\begin{array}{c}\text { Number of Pixels } \\
\text { within } \\
\text { the Range Mode to } \\
\text { Max }\end{array}$ & $\%$ \\
\hline \multirow{19}{*}{$\begin{array}{l}\text { Control } \\
\text { Group }\end{array}$} & 1 & 8057 & 2104 & 26,11 \\
\hline & 2 & 7414 & 1986 & 26,79 \\
\hline & 3 & 10972 & 4737 & 43,17 \\
\hline & 4 & 12856 & 4525 & 35,20 \\
\hline & 5 & 14813 & 4690 & 31,66 \\
\hline & 6 & 10753 & 2774 & 25,80 \\
\hline & 7 & 9144 & 5079 & 55,54 \\
\hline & 8 & 10961 & 4863 & 44,37 \\
\hline & 9 & 11531 & 6587 & 57,12 \\
\hline & 10 & 16445 & 9582 & 58,27 \\
\hline & 11 & 11287 & 5184 & 45,93 \\
\hline & 12 & 10706 & 6459 & 60,33 \\
\hline & 13 & 9883 & 2570 & 26,00 \\
\hline & $\overline{14}$ & 12221 & 5261 & 43,05 \\
\hline & 15 & 9046 & 5688 & 62,88 \\
\hline & 16 & 10209 & 2342 & 22,94 \\
\hline & 17 & 10467 & 3913 & 37,38 \\
\hline & 18 & 11969 & 5976 & 49,93 \\
\hline & \multicolumn{3}{|c|}{ Average \% of Control Group } & 41,80 \\
\hline \multirow{14}{*}{$\begin{array}{l}\text { Patient } \\
\text { Group }\end{array}$} & 1 & 14238 & 3660 & 25,71 \\
\hline & 2 & 15852 & 5743 & 36,23 \\
\hline & 3 & 14238 & 10001 & 70,24 \\
\hline & 4 & 9155 & 3856 & 42,12 \\
\hline & 5 & 9673 & 1664 & 17,20 \\
\hline & 6 & 14064 & 2656 & 18,89 \\
\hline & 7 & 11105 & 3781 & 34,05 \\
\hline & 8 & 14081 & 5422 & 38,51 \\
\hline & 9 & 11868 & 7201 & 60,68 \\
\hline & 10 & 11840 & 2876 & 24,29 \\
\hline & 11 & 14216 & 4818 & 33,89 \\
\hline & 12 & 7948 & 2367 & 29,78 \\
\hline & 13 & 11149 & 6043 & 54,20 \\
\hline & \multicolumn{3}{|c|}{ Average $\%$ of Patient Group } & 37,37 \\
\hline
\end{tabular}

The control group has more pixels driven to one within the knee region, or a higher percentage covering the knee region, since its mode temperature is generally lower than that of the patient group. Therefore, from the table above, it is possible to state that more pixels fall in the range mode to maximum temperature for the control group than for the patient group. 
MATLAB's ranksum test is then applied to the control and to the patient percentage matrix distributions to see if they are statistically different at a significance level of $5 \%$. The ranksum test outputs are $h$ equals 0 and $p$ equals 0.2888. The null hypothesis is not rejected; therefore the percentage of the joint that has warmer pixels and/or hot spots cannot be used to statistically discriminate between the patient and control group. In conclusion, the binary image can only be used as a visual tool to discriminate between control and patient TIR image.

To complete the overall analysis of objective one, a cluster chart is created to identify a potential threshold that could distinguish between the control samples and the patient samples. Since the $2^{\text {nd }}$ MCP's mode/max matrix gave great statistical significant results (using the ranksum test above), its matrix element values were used for the cluster chart. The graph below is the cluster chart of the mode/max matrix of the $2^{\text {nd }}$ MCP joint of the control and patient group. 


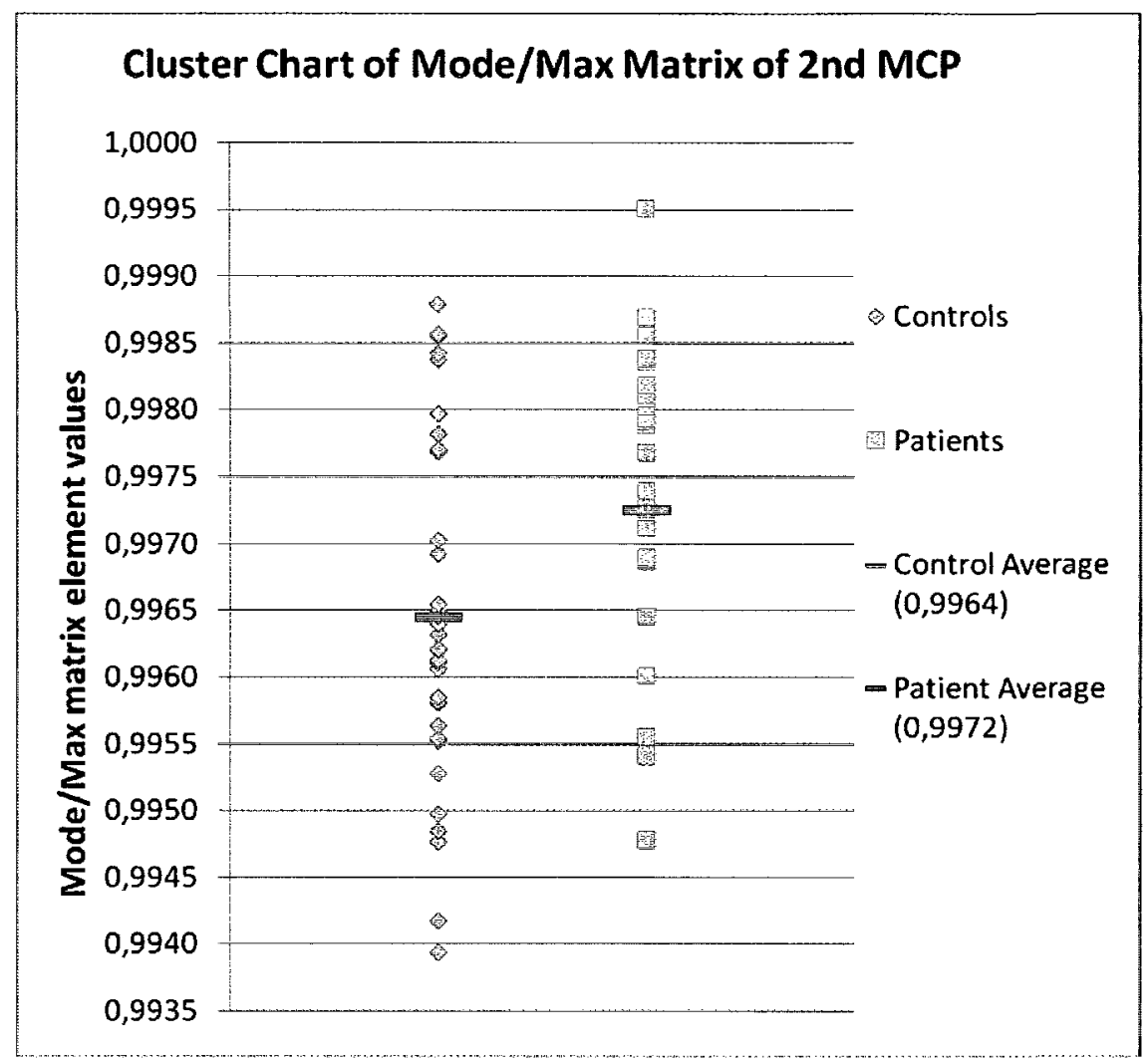

Figure 4-2: Cluster Chart of Mode/Max Matrix of the 2nd MCP of the Control and the Patient Groups

From this graph, one can see that the highest density of the control group lies around 0,99625 and the highest density of the patient group lies around 0,99825 . The averages do not represent the highest density for both groups since many outliers with extreme values do not follow the trend of their respective groups. Explanation for these outliers can be a failure to comply with protocol procedures, the influence of medication or a bad imaging session (imaging technician's error). Therefore, using the high density groupings for the patients and the control subjects, a potential threshold can be seen at around 0.997 to discriminate between the groups. 
As this project continues to gather more samples, this threshold will become more and more evident, eliminating the outliers and putting more emphasis on the highest density region of each group. With the small sample set that this thesis is using, an exact value cannot be determined; however, the cluster graph clearly shows that a density trend is forming to see that a threshold will be able to be identified in the future with additional samples.

The results of the matrices extracted from the raw measurements of the TIR image, the visual highlights of the warmer and/or hot spot regions covering the joint of the binary images, and the potential thresholds of the cluster graphs show that it is possible to conclude that the first objective has been satisfied. There are many matrices (listed above) that are able to statistically differentiate at a $5 \%$ significance level between the control distribution and the patient distribution. Histograms of these matrices graphically confirm this differentiating capacity. The visual binary images clearly show that a patient could be identified with the presence of hot spots and by a negative temperature gradient from the patella of the knee to the inner flexed knee. The cluster graph demonstrates that a threshold is forming to discriminate between the control samples and the patient samples. 
4.2.2 Second Objective - Identify the Best Joints that Best Determines whether a Participant has RA

From the normality Lilliefors tests completed in the first objective, it is assumed that all matrices extracted from the raw measurements are not normally distributed. Therefore, to complete the second goal of the thesis, non-parametric hypothesis tests are used once again. The same ranksum tests are applied to the matrix distributions of the control group and the patient group as explained in the first objective. The results are displayed once again in Table 4.3 from the first objective section.

From the ranksum hypothesis test results displayed in Table 4.3 it is possible to see that the matrices of the $2^{\text {nd }}$ PIP, the $3^{\text {rd }}$ PIP and the wrist cannot differentiate between the control distributions and the patient distributions. Only the kurtosis matrix of the $3^{\text {rd }}$ PIP shows statistically significant results. The joints that best distinguish between the control and patient groups are the $2^{\text {nd }} \mathrm{MCP}$, the $3^{\text {rd }}$ MCP and the knee, since they have many matrices that discriminate between the control and patient distribution at a significance level of $5 \%$.

Refer once again to Appendix $E$ to graphically view the histograms of the matrices of the best representative joints. These histograms show that within the $2^{\text {nd }} \mathrm{MCP}, 3^{\text {rd }} \mathrm{MCP}$ and knee, the matrices of the patients are shifted differently compared to the matrices of the control subjects. The bins containing the most elements for the control group is never the same as the bins containing the most elements for the patient group. One histogram example is shown below. 


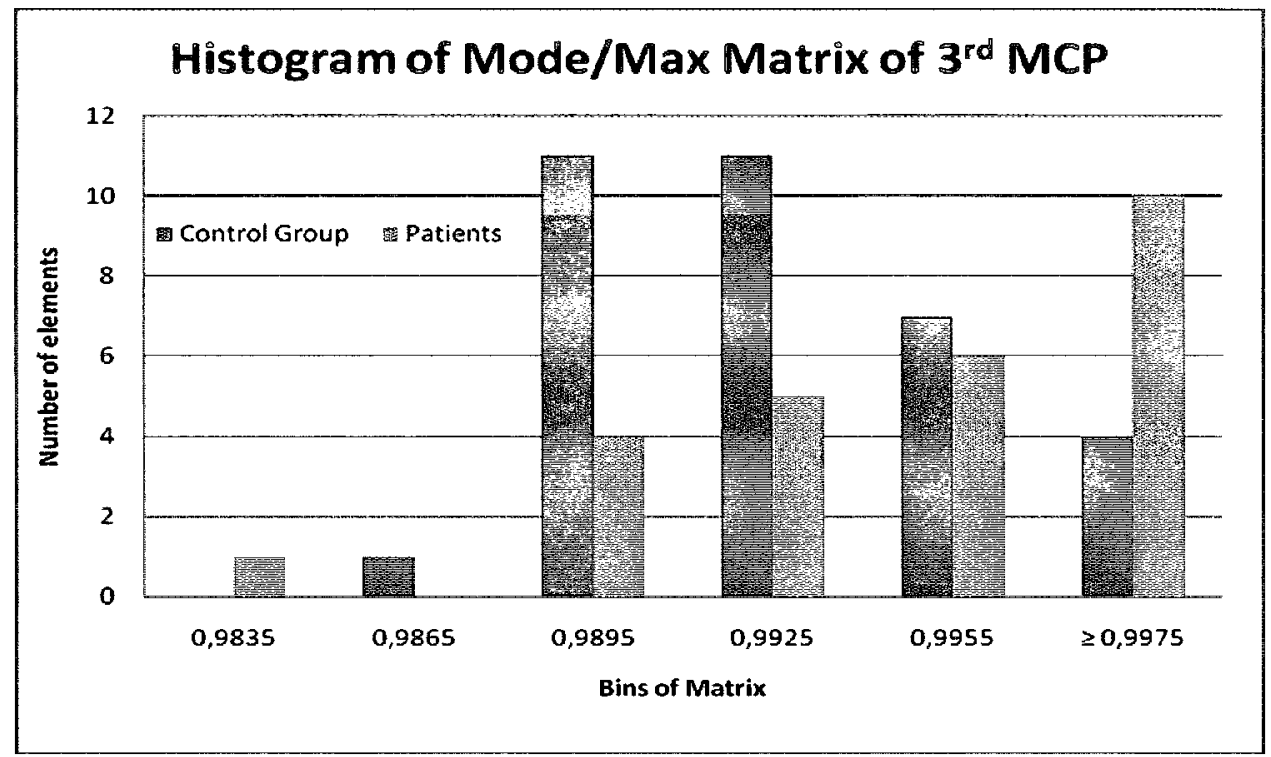

Figure 4-3: Histogram of the Mode/Max Matrix of the 3rd MCP

Again, this histogram shows that the control subjects' $3^{\text {rd }}$ MCP mode/max matrix is shifted to the left, whereas the patients' mode/max matrix is shifted to the right. The bin containing the most elements in the control's matrix is the 0.9895 bin whereas the bin containing the most elements in the patient's matrix is greater than 0.9975 . It is clear from the histogram above that the distributions of the mode/max matrix of the $3^{\text {rd }}$ MCP can distinguish between the control and patient groups.

Several factors could explain the fact that the PIP joints do not show better statistical results is its small area. The average amount of pixels within the PIP joint is 435 pixels compared to 12243 pixels in the knee joint. This smaller region makes it more difficult to observe an abnormal thermal distribution and to observe the presence of irregular hot/cold spots. Furthermore, the wrist is taken from the posterior view of the frontal plane. This view might not result in the best 
representation of the intra-articular temperature because of barriers that prevent the dissipation of heat to the surface of the skin - for example, hair (especially in men participants) and the pisiform bone. This view is chosen over the anterior view of the frontal plane because in the anterior view the carpal tunnel, containing nerves and ligaments, covers the joint creating more of a barrier for the intra-articular heat to dissipate to the surface of the skin. Other views could be explored to find a better representation of the intra-articular joint of the wrist.

The hypothesis stated in chapter 3 that the MCPs and the knees are the best joints in a clinical setting to best determine if a participant has or does not have RA is therefore accepted. The second objective is therefore satisfied since the matrices of the MCPs and the knees were able to discriminate between the control group and the patient group.

\subsubsection{Third Objective - Identify the Matrix that Best Assesses the Presence or} Absence of RA

The same ranksum test results are used to satisfy the third objective of this thesis. Referring to the results displayed in Table 4.3, it is possible to identify which matrices extracted from the raw measurements of the TIR images best distinguish between the control and patient groups. Using the best representative joints found in objective two, the mathematical matrices that best rendered statistical differences between both groups are the maximum temperature minus the minimum temperature (max-min), the variance (standard 
deviation squared) and the ratio between the mode and maximum temperatures (mode/max). The first and second mathematical matrices - max-min and variance - represent the range and the dispersion of the thermal distribution within the joint. The third mathematical matrix - mode/min - can represent abnormal hot regions that characterize RA disease.

Appendix $\mathrm{E}$ graphically displays the histograms of the best representative matrices of the best representative joints (objective two). These histograms show that max-min, mode/max and variance matrices of the $2^{\text {nd }} M C P, 3^{\text {rd }} M C P$ and knee can distinguish between the patient and control groups. Their respective histograms are shifted differently and their bins containing the most elements are always different and far apart. One histogram example is shown below.

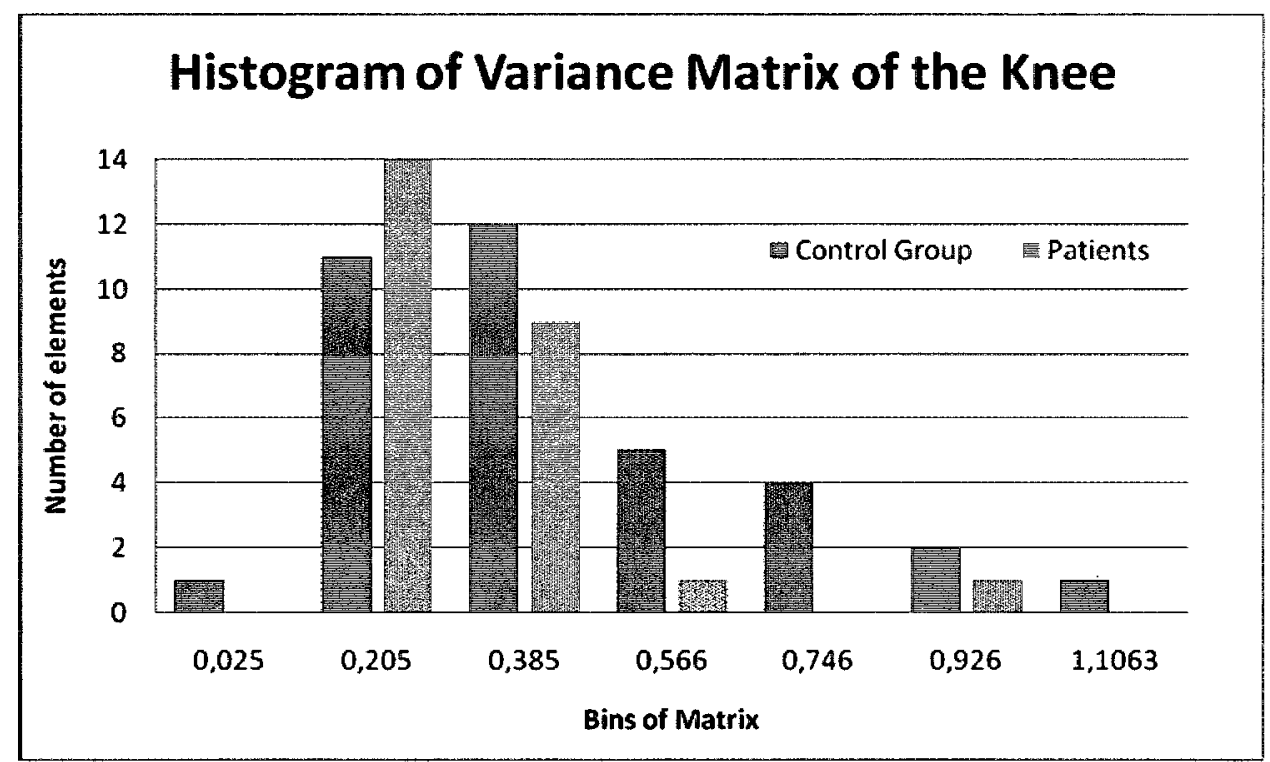

Figure 4-4: Histogram of the Variance Matrix of the Knee 
This histogram shows that the control subject's knee variance matrix is shifted to the right whereas the patient's variance matrix is shifted to the left. The bin containing the most elements in the control's matrix is the 0.385 bin whereas the bin containing the most elements in the patient's matrix is the $0.205 \mathrm{bin}$. It is clear from the histogram above that the distributions of the variance matrix can distinguish between the control and patient groups.

All the histograms of the best representative matrices found in Appendix $E$ had outlier elements found in the last bin. In all cases but one, the outliers have extremely high values that skew the shape of the histogram. These outliers could be the result of a failure to comply to all the TIR imaging protocols (such as applying lotions to the skin, doing physical activity prior to the session), or a failure to comply to recording protocols (such as body parts touching other body parts causing heat transfers that are reflected at the surface of the skin). Another variable that could have caused outlier elements in the patient group matrices is the influence of first or second line medications taken by the patient. These medications may be in their trial mode, or may be taken because of a flare period. The influence and effect of these first and second line medications are unknown at this stage of the study. In future work on this project, it is expected that these outliers will have less of an impact on the matrix since more participants will be imaged to increase the sample size of the study.

The hypothesis stated in the previous chapter that dispersion matrices will statistically distinguish between the control and patient group can be accepted. In addition to the dispersion matrices - variance and max-min - is the mode/max 
matrix that can also statistically differentiate between both group distributions. This can be explained by the fact that abnormal TIR images are not only characterised by their non-uniform thermal distribution, but also by the presence of hot spots. The presence of a hot spot increases the value of the mode temperature, thus making the patients mode/max ratio generally smaller than that of the control group. The third objective is therefore satisfied since the mode/max, the variance and the max-min matrices of the MCPs and the knees were able to discriminate between the control group and the patient group.

The matrix distributions from Table 4-3 that did not reject the hypothesis tests (in others words, they rendered an $h$ value of 0 ) cannot be used to discriminate between the control and the patient groups. They could not reject the null hypothesis that two matrices are independent samples from identical continuous distributions with equal medians. Therefore the control matrix and the patient matrix have identical distributions with equal medians. Since the matrices are not statistically significant in the context of differentiating between the control subjects and the patients, they cannot be used to answer objectives 4 and 5. Only the matrices that rejected Matlab's ranksum hypothesis test at a significance level of 0.05 can be explored for objectives 4 and 5 . Those matrices are the variance, the max-min and the mode/max matrices. Utilising these matrices will statistically validate the results obtained from objectives 4 and 5 . 


\subsubsection{Fourth Objective - Classify RA Patients into Three Severity Groups}

First, the patients were classified into three RA severity groups - high, moderate and low. Dr. Jacob Karsh is the specialist who identified each patient within the RA group. His classification is as follows:

Table 4-7: Physician's Assessment of Each Patient and their Corresponding RA Severity Group

\begin{tabular}{|c|c|c|c|}
\hline Patient \# & Sexe & $\begin{array}{c}\text { RA } \\
\text { Severity }\end{array}$ & Age \\
\hline $\mathbf{1}$ & M & Low & 65 \\
\hline $\mathbf{2}$ & F & Low & 58 \\
\hline $\mathbf{3}$ & M & High & 84 \\
\hline $\mathbf{4}$ & F & Low & 64 \\
\hline $\mathbf{5}$ & F & Low & 44 \\
\hline $\mathbf{6}$ & M & Low & 71 \\
\hline $\mathbf{7}$ & F & High & 79 \\
\hline $\mathbf{8}$ & F & Low & 40 \\
\hline $\mathbf{9}$ & M & Low & 68 \\
\hline $\mathbf{1 0}$ & F & Moderate & 69 \\
\hline $\mathbf{1 1}$ & F & Moderate & 67 \\
\hline $\mathbf{1 2}$ & F & Low & 53 \\
\hline 13 & F & Moderate & 64 \\
\hline
\end{tabular}

The patients in the High group are ranked 1 and 2 . The patients in the Moderate group were ranked 3,4 and 5. The rest of the patients in the Low group were ranked from 6 to 13 .

Second, the patient's $2^{\text {nd }}$ MCP mode/max matrix is selected for this test since its $p$-value is very small compared to the variance and max-min matrices. Its $p$-value is 0.0105 and therefore should strongly represent the degree of severity of the illness of the patients. The table below shows the patient's $2^{\text {nd }}$ MCP mode/max matrix elements ranked from highest to lowest according to their 
values. The highest value is given a rank of 1 and the lowest value is given a rank of 13.

Table 4-8: Ranking of the Patient's 2nd MCP Mode/Max Matrix

\begin{tabular}{|c|c|c|}
\hline $\begin{array}{c}\text { Patient } \\
\#\end{array}$ & $\begin{array}{c}\text { Mode/Max } \\
\text { Matrix } \\
\text { Elements }\end{array}$ & $\begin{array}{c}\text { Ranking of } \\
\text { Mode/Max } \\
\text { (from Highest to } \\
\text { Lowest) }\end{array}$ \\
\hline $\mathbf{1}$ & 0,99541 & 12 \\
\hline $\mathbf{2}$ & 0,997933 & 4 \\
\hline $\mathbf{3}$ & 0,998104 & 2 \\
\hline $\mathbf{4}$ & 0,997877 & 5 \\
\hline $\mathbf{5}$ & 0,998019 & 3 \\
\hline $\mathbf{6}$ & 0,990813 & 13 \\
\hline $\mathbf{7}$ & 0,997116 & 8 \\
\hline $\mathbf{8}$ & 0,997264 & 7 \\
\hline $\mathbf{9}$ & 0,99646 & 10 \\
\hline $\mathbf{1 0}$ & 0,996009 & 11 \\
\hline $\mathbf{1 1}$ & 0,996868 & 9 \\
\hline $\mathbf{1 2}$ & 0,997396 & 6 \\
\hline $\mathbf{1 3}$ & 0,998684 & 1 \\
\hline
\end{tabular}

The Spearman Correlation Coefficient is then used to see the extent to which the physician's ranking of severity correlates with the mode/max matrix ranking of the patient (Salvatore, 2002). The Spearman Correlation Coefficient states:

$$
\text { SpearmanCoeff }=1-\frac{6 \sum D^{2}}{n\left(n^{2}-1\right)}
$$

where $D$ is the difference in rankings and $n$ is the number of samples. The closer the coefficient to 1 the more correlated the rankings. The table below displays the calculations of the Spearman Correlation Coefficient between the physician ranking and the matrix ranking. 
Table 4-9: Spearman Correlation Coefficient Calculation Between the Physician Ranking and the Matrix Ranking

\begin{tabular}{|c|c|c|c|c|c|}
\hline $\begin{array}{c}\text { Patien } \\
\mathbf{t} \#\end{array}$ & $\begin{array}{c}\text { Mode/Max } \\
\text { Matrix } \\
\text { Elements }\end{array}$ & $\begin{array}{c}\text { Mode/Max } \\
\text { Matrix } \\
\text { ranking of } \\
\text { Patients }\end{array}$ & $\begin{array}{c}\text { Physician } \\
\text { Ranking of } \\
\text { Patients by } \\
\text { RA } \\
\text { Severity }\end{array}$ & $\mathbf{D}$ & $\mathbf{D}^{\mathbf{2}}$ \\
\hline $\mathbf{1}$ & 0,99541 & 12 & 12 & 0 & 0 \\
\hline $\mathbf{2}$ & 0,997933 & 4 & 7 & 3 & 9 \\
\hline $\mathbf{3}$ & 0,998104 & 2 & 1 & -1 & 1 \\
\hline $\mathbf{4}$ & 0,997877 & 5 & 8 & 3 & 9 \\
\hline $\mathbf{5}$ & 0,998019 & 3 & 6 & 3 & 9 \\
\hline $\mathbf{6}$ & 0,990813 & 13 & 13 & 0 & 0 \\
\hline $\mathbf{7}$ & 0,997116 & 8 & 2 & -6 & 36 \\
\hline $\mathbf{8}$ & 0,997264 & 7 & 10 & 3 & 9 \\
\hline $\mathbf{9}$ & 0,99646 & 10 & 11 & 1 & 1 \\
\hline $\mathbf{1 0}$ & 0,996009 & 11 & 5 & -6 & 36 \\
\hline $\mathbf{1 1}$ & 0,996868 & 9 & 4 & -5 & 25 \\
\hline $\mathbf{1 2}$ & 0,997396 & 6 & 9 & 3 & 9 \\
\hline $\mathbf{1 3}$ & 0,998684 & 1 & 3 & 2 & 4 \\
\hline
\end{tabular}

The sum of $D^{2}$ is 148 and $n$ 's value is 13 , giving a Spearman Correlation Coefficient of 0.593 . Since the coefficient is above 0.5 , it is possible to state that mode/max matrix elements for the $2^{\text {nd }} \mathrm{MCP}$ joint are strongly correlated to the physician's severity classification.

The same analysis is repeated with the patient's $3^{\text {rd }}$ MCP mode/max matrix ( $p$-value of 0.0212 ), to see if its ranked elements highly correlate with the physician's severity classification. The Spearman Correlation Coefficient calculations resulted in a $D^{2}$ of 192 giving a coefficient of 0.473 . Since the coefficient is lower than 0.5 , it is possible to state that the mode/max matrix elements for the $3^{\text {rd }}$ MCP joint are correlated more weakly to the physician's severity classification. 
The same analysis is repeated one more time with the patient's Knee variance matrix ( $p$-value of 0.0341 ), to observe whether the ranked elements highly correlate with the physician's severity classification. The Spearman Correlation Coefficient calculations resulted in a $D^{2}$ of 169 giving a coefficient of 0.536. Since the coefficient is above 0.5 , it is possible to state that the knee is also correlated strongly to the physician's severity classification. However, it is important to note, that the knee's correlation coefficient was lower than the $2^{\text {nd }}$ MCP's correlation coefficient. Therefore, the strongest correlation found was the correlation of the patient's $2^{\text {nd }}$ MCP mode/max matrix; which correctly classified the patients within their severity group at a Spearman Correlation Coefficient of 0.593. The graph below graphically demonstrates with increased RA severity there is an increase in the average mode/max value.

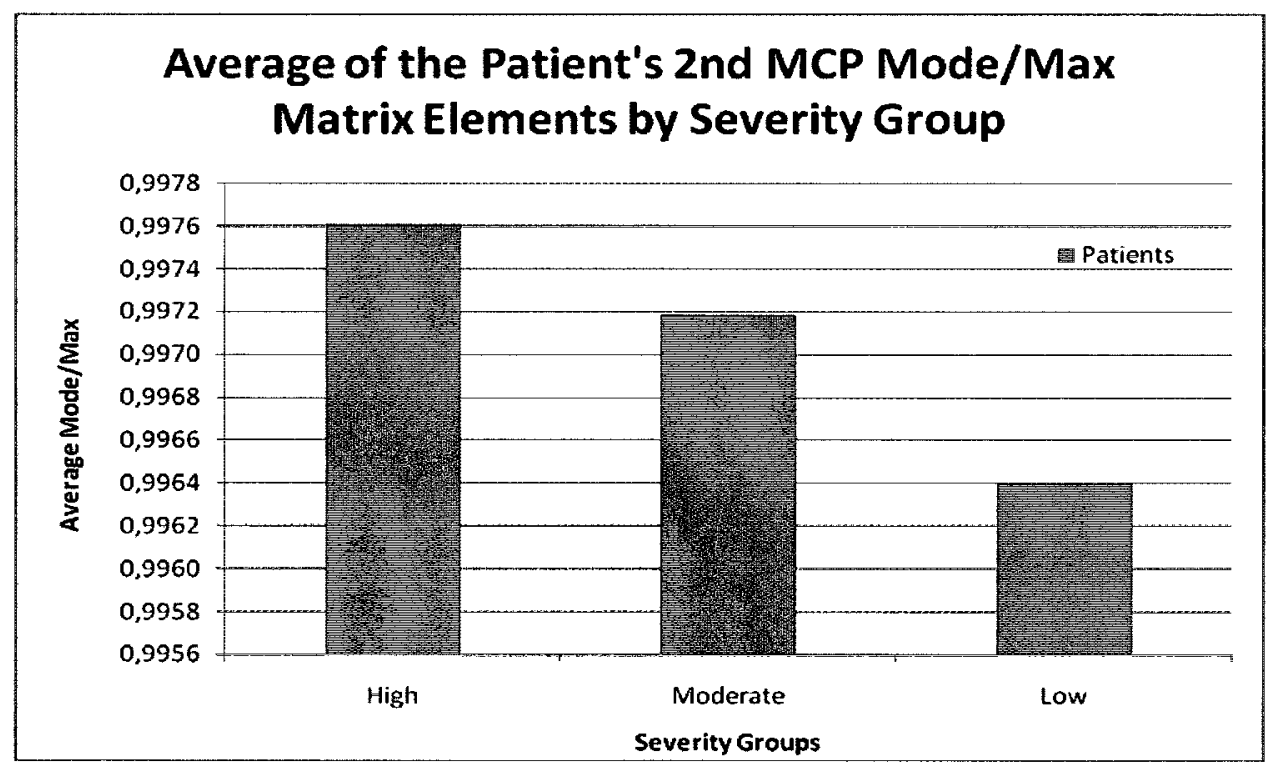

Figure 4-5: Average of the Patient's 2nd MCP Mode/Max Matrix Elements by Severity Group 
The graph therefore confirms that the patients can be classified into three severity groups using the patient's $2^{\text {nd }} \mathrm{MCP}$ mode/max matrix ranking. The fourth objective is therefore satisfied, since the patient's $2^{\text {nd }} M C P$ mode/max matrix ranking highly correlations with the severity rankings of the physician with a Spearman Correlation Coefficient greater than 0.5 .

\subsubsection{Fifth Objective - Obsenve the Effect of Age on Thermal IR Matrices}

In an attempt to explain the small overlap of elements between the patients and the older control subjects observed in the histograms of Appendix $E$, the effect of age on the matrices was studied. The Spearman Correlation Coefficient is used to see the extent to which the control subjects' age correlates with the mode/max matrix of the $2^{\text {nd }} M C P$ of the control group. Again, the control's $2^{\text {nd }}$ MCP mode/max matrix was chosen for its low $p$-value ( $p$ equals $0.0105)$ and its success in distinguishing between the control and patient groups (objectives 1 through 3 ). The table below shows the control subjects and their corresponding age groups. 
Table 4-10: Control Subjects with their Corresponding

\begin{tabular}{|c|c|c|}
\hline Control \# & Sexes & Age Group \\
\hline 1 & F & $20-30$ \\
\hline 2 & F & $10-20$ \\
\hline 3 & F & $20-30$ \\
\hline 4 & M & $20-30$ \\
\hline $\mathbf{6}$ & M & $20-30$ \\
\hline $\mathbf{6}$ & M & $50-60$ \\
\hline 8 & F & $50-60$ \\
\hline $\mathbf{1 0}$ & M & $40-50$ \\
\hline 11 & M & $60-70$ \\
\hline 12 & F & $60-70$ \\
\hline 13 & M & $20-30$ \\
\hline 14 & M & $20-30$ \\
\hline 15 & F & $20-30$ \\
\hline 16 & M & $30-40$ \\
\hline 17 & F & $20-30$ \\
\hline 18 & F & $20-30$ \\
\hline & M & $60-70$ \\
\hline
\end{tabular}

First, the control subjects are ranked according to their age group. The control subjects in the 60-70 age group are ranked 1,2, 3 and 4. The control subjects in the 50-60 age group are ranked 5 and 6 ; and so on.

Second, the control subjects are ranked according to their $2^{\text {nd }}$ MCP mode/max matrix value. The table below shows the control subject's $2^{\text {nd }} M C P$ mode/max matrix elements ranked from highest to lowest according to their values. The highest value is given a rank of 1 and the lowest value is given a rank of 18. 
Table 4-11: Ranking of the Control's 2nd MCP Mode/Max Matrix

\begin{tabular}{|c|c|c|}
\hline $\begin{array}{c}\text { Control } \\
\#\end{array}$ & $\begin{array}{c}\text { Mode/Max } \\
\text { Matrix } \\
\text { Elements }\end{array}$ & $\begin{array}{c}\text { Ranking of } \\
\text { Mode/Max } \\
\text { (from Highest to } \\
\text { Lowest) }\end{array}$ \\
\hline $\mathbf{1}$ & 0,997819 & 5 \\
\hline $\mathbf{2}$ & 0,996318 & 9 \\
\hline $\mathbf{3}$ & 0,99797 & 4 \\
\hline $\mathbf{4}$ & 0,996923 & 7 \\
\hline $\mathbf{5}$ & 0,996463 & 8 \\
\hline $\mathbf{6}$ & 0,996196 & 11 \\
\hline $\mathbf{7}$ & 0,996057 & 13 \\
\hline $\mathbf{8}$ & 0,994762 & 17 \\
\hline $\mathbf{1 0}$ & 0,998375 & 3 \\
\hline $\mathbf{1 1}$ & 0,995535 & 15 \\
\hline $\mathbf{1 2}$ & 0,998791 & 1 \\
\hline $\mathbf{1 3}$ & 0,995277 & 16 \\
\hline $\mathbf{1 4}$ & 0,996206 & 10 \\
\hline $\mathbf{1 5}$ & 0,997702 & 6 \\
\hline 16 & 0,996122 & 12 \\
\hline $\mathbf{1 7}$ & 0,995632 & 14 \\
\hline $\mathbf{1 8}$ & 0,998566 & 2 \\
\hline
\end{tabular}

The same equation (4.2) is used to calculate the Spearman Correlation Coefficient between the ranking of the control subjects done by age group and the ranking of the control subjects done by the values of the $2^{\text {nd }}$ MCP's mode/max matrix elements. The table below displays the calculations of the Spearman Correlation Coefficient. 
Table 4-12: Spearman Correlation Coefficient Calculation

Between the Age Ranking and the Matrix Ranking

\begin{tabular}{|c|c|c|c|c|c|}
\hline $\begin{array}{c}\text { Control } \\
\#\end{array}$ & $\begin{array}{c}\text { Mode/Max } \\
\text { Matrix }\end{array}$ & $\begin{array}{c}\text { Mode/Max } \\
\text { Matrix } \\
\text { ranking of } \\
\text { Control } \\
\text { subjects }\end{array}$ & $\begin{array}{c}\text { Ranking of } \\
\text { Control } \\
\text { subject by } \\
\text { Age Group }\end{array}$ & $\mathbf{D}$ & $\mathbf{D}^{\mathbf{2}}$ \\
\hline $\mathbf{1}$ & 0,997819 & 5 & 10 & 5 & 25 \\
\hline $\mathbf{2}$ & 0,996318 & 9 & 17 & 8 & 64 \\
\hline $\mathbf{3}$ & 0,99797 & 4 & 9 & 5 & 25 \\
\hline $\mathbf{4}$ & 0,996923 & 7 & 12 & 5 & 25 \\
\hline $\mathbf{5}$ & 0,996463 & 8 & 13 & 5 & 25 \\
\hline $\mathbf{6}$ & 0,996196 & 11 & 4 & -7 & 49 \\
\hline $\mathbf{7}$ & 0,996057 & 13 & 5 & -8 & 64 \\
\hline $\mathbf{8}$ & 0,994762 & 17 & 6 & -11 & 121 \\
\hline $\mathbf{1 0}$ & 0,998375 & 3 & 2 & -1 & 1 \\
\hline $\mathbf{1 1}$ & 0,995535 & 15 & 15 & 0 & 0 \\
\hline $\mathbf{1 2}$ & 0,998791 & 1 & 8 & 7 & 49 \\
\hline $\mathbf{1 3}$ & 0,995277 & 16 & 16 & 0 & 0 \\
\hline $\mathbf{1 4}$ & 0,996206 & 10 & 7 & -3 & 9 \\
\hline $\mathbf{1 5}$ & 0,997702 & 6 & 11 & 5 & 25 \\
\hline $\mathbf{1 6}$ & 0,996122 & 12 & 14 & 2 & 4 \\
\hline $\mathbf{1 7}$ & 0,995632 & 14 & 3 & -11 & 121 \\
\hline $\mathbf{1 8}$ & 0,998566 & 2 & 1 & -1 & 1 \\
\hline & & & & & \\
\hline
\end{tabular}

The sum of $D^{2}$ is 608 and $n$ 's value is 18 , giving a Spearman Correlation Coefficient of 0.255 . Since the coefficient is very low it is possible to state that age is associated weakly with the mode/max matrix of the control group. However, a coefficient of 0.255 is enough to explain the small overlap that is seen between the matrices of the control and the patient group.

The graph below shows that an increased age does not necessarily lead to a higher average mode/max value. The average mode/max value is not highly correlated to age. 


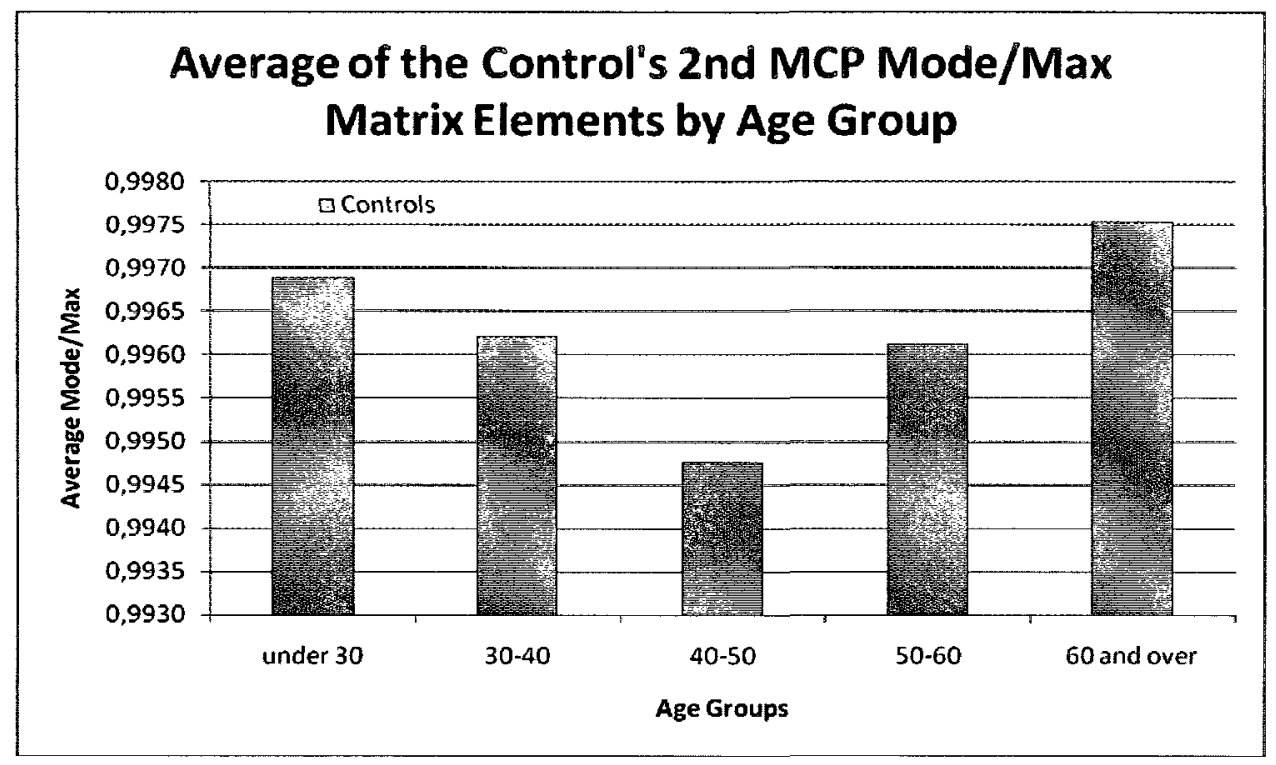

Figure 4-6: Average of the Control's 2nd MCP Mode/Max Matrix Elements by Age Group

From the graph above, it is possible to visually see that the correlation is low between the control subject's age and their average mode/max matrix elements. The older age group does not necessarily equate to a higher mode/max reading from the TIR image. However, the 0.255 value can be due to the 60 and over age group, where it is obvious that the average TIR's ratio of mode/max is above the rest of the age groups. Therefore, the over 60 age group may explain the small overlap that is seen between the patient and control samples in the histograms of Appendix E.

An interesting finding is that, the mode/max matrix of the $2^{\text {nd }} \mathrm{MCP}$ within the patient group had a Spearman Correlation Coefficient of 0.402 with the age of the patients. This suggests that in the presence of disease, a degenerative factor, the severity RA amplifies the abnormal results of the RA patients. 
The pathophysiology of RA can support the statement that with age, a degenerative factor can affect the outcome within the patient group. As previously explained in the first chapter, severe RA causes joint deformity as well as bone destruction. Age and the overuse of the body's joints will make the joint more susceptible to bone destruction and joint deformity. These physiological changes will increase the inflammation of a joint and thus increase the subcutaneous temperature of an affected RA joint.

The firth objective is met, since the correlation between the ranking of the control subjects by their $2^{\text {nd }} \mathrm{MCP}$ mode/max matrix elements and by their age group was studied. It was possible to observe that age does not have much of an effect on the matrices of the control group. However, the Spearman Correlation Coefficient is 0.402 between the ranking of the patients by their $2^{\text {nd }}$ MCP mode/max matrix elements and by their age group. Although the Spearman Coefficient is not greater than 0.5 , it is high enough to suggest that there is a weak age-degenerative factor that influences the RA measurements of the patients.

The final chapter of this thesis states the conclusions that can be made from the results and the analysis above, the contributions made to knowledge and suggests future work. 


\section{CHAPTER 5}

\section{CONCLUSION}

This chapter states the contributions to knowledge of this thesis work. It will end with some proposed future work.

\subsection{Contributions to Knowledge}

The first contribution to knowledge was the completion of objective one, that is, the thesis showed that the ratio/difference/dispersion matrices represent correctly the thermal distributions on the surface of the skin. These matrices can distinguish between the temperature distribution of the control group and of the patient group. Moreover, a new method of visually observing the thermal distribution and the presence of hot regions on the knee is presented. Binary images are created using MATLAB's roicolor function and they visually illustrate a difference between the control and patient TIR images. The patients' binary images have negative temperature gradients from the patella to the inside of the knee and have abnormal hot regions present. Finally, by representing a highly statistical significant matrix (the $2^{\text {nd }} M C P$ 'S mode/max matrix) in a cluster graph this thesis demonstrates a threshold to be determined to clearly discriminate 
between the control and patient groups. Distinct highly dense regions were visible in the patient cluster and in the control cluster.

The second contribution to knowledge was the identification of the joints to examine in a clinical setting that best determine if a participant has or does not have RA. The best joints are the $2^{\text {nd }} \mathrm{MCP}$, the $3^{\text {rd }} \mathrm{MCP}$ and the knee. These joints statistically showed at a significance level of $5 \%$ that they were able to differentiate between the control's matrix measurements and the patient's matrix measurements. This was proven using the non-parametric ranksum and Kruskal-Wallis tests.

The third contribution to knowledge was the identification of the best mathematical calculations of the data to assess the difference between a control and a RA patient. A wide variety of dispersion, ratio and difference matrix distributions were analysed and statistically tested. The best representative matrices of RA are the matrices that characterize the dispersion measurements of the distribution and the matrix ratios that can distinguish between the presence or absence of high temperature regions (via the mode temperature). The best dispersion measurement matrices are the variance and the max-min matrices; and the best matrix representing high temperatures is the mode/max matrix. This was again demonstrated using the non-parametric ranksum and KruskalWallis tests.

The fourth contribution to knowledge was to succeed in classifying the RA patients into three severity groups using the best representative matrix (objective three) of the best representative joint (objective two). The patient's $2^{\text {nd }}$ MCP 
mode/max matrix was proven to correlate strongly with the physician's RA severity ranking of the patients; thus proving that the elements within this matrix could be used to classify the RA patients into high, moderate and low severity groups. This was proven using the Spearman Correlation Coefficient.

The final contribution to knowledge was the study of the effect of age on the thermal IR measurements and the resulting calculated matrices. The thesis found that within the control group, age correlates weakly to the $2^{\text {nd }}$ MCP'S mode/max matrix. It is possible to state that age does not greatly affect the extracted measurements of the control's TIR images. Within the patient group, the thesis found that there is a weak but noticeable correlation between the patient's age and their measurements results. Based on this, the thesis found an age-degenerative factor in the patients' RA measurements.

\subsection{Future Work}

To further the thermal infrared imaging work of the MIRG's research team, it would be necessary to collect data on more patients to increase the sample size of the database. During these imaging sessions, it is recommended to have fixed cameras for imaging to standardize the distance and the zoom of the recorded images. This would allow the amount of pixels within each ROI to be identical in order to more easily compare the analysis results.

Applying image processing techniques to the TIR images would have been useful to eliminate surface effects, such as hair (especially within the ROls 
of male participants' knees and wrists). Filtering and image enhancing techniques could be explored to improve the data extraction for analysis.

Further analysis should be done to refine the assessment of age on the measurements and the calculated matrices. The participants in the control group should also be chosen to represent the RA patient group with respect to age. In this thesis, $70 \%$ of the patients imaged were above the age of 60 and only $22 \%$ of the control subjects were above that age.

In the future, it would be important that patients be assessed by the physician on the day of the imaging session or soon after/before the imaging session. This would provide necessary medical information with regards to their RA state - "are they in a flare period, are their medications effective in controlling inflammation", etc. This additional information would serve to further classify patients by severity and would better explain outliers or extreme results. This thesis did not compare IR results with a recent physician's physical examination and therefore did not have all the information to complete an in-depth analysis of the pathophysiology and corresponding RA samples.

Repetitive imaging sessions should also be done to assess the effect of medication on the intra-articular temperature and disease progression. This effect can be assessed by doing repetitive imaging sessions on the same patients during a determined treatment period to quantify via TIR the pain and inflammation of the treated joints. A longitudinal comparison can be made, comparing the thermal IR distribution of the first set of images with the successive ones. 
Correlation of IR imaging results should be statistically compared to RA diagnosis gold standards such as MRI and HRUS, which are tools that can correctly classify the normal versus the RA patient. This would further solidify the conclusions stated in this thesis. This comparison would also assess if IR technology can detect inflammation as early on in the disease as MRI and HRUS. As discussed in the introductory chapter, detecting inflammation early would assist in diagnosing RA before the manifestation of clinical symptoms and improve the prognosis of the patient.

As previously stated, this thesis is the first step of a research project that will incorporate multi-spectral imaging to IR imaging as a diagnostic tool for RA disease. This research project will therefore continue with the following objectives. First, incorporating Christophe Herry's work to the IR system would allow the incorporation of automated segmentation and automated extraction of ROI. Future work would also include an automated detection of anomalies within the selected $\mathrm{ROI}$ and a classification mechanism between normal and abnormal. The automated system that is envisioned is shown in the figure below.

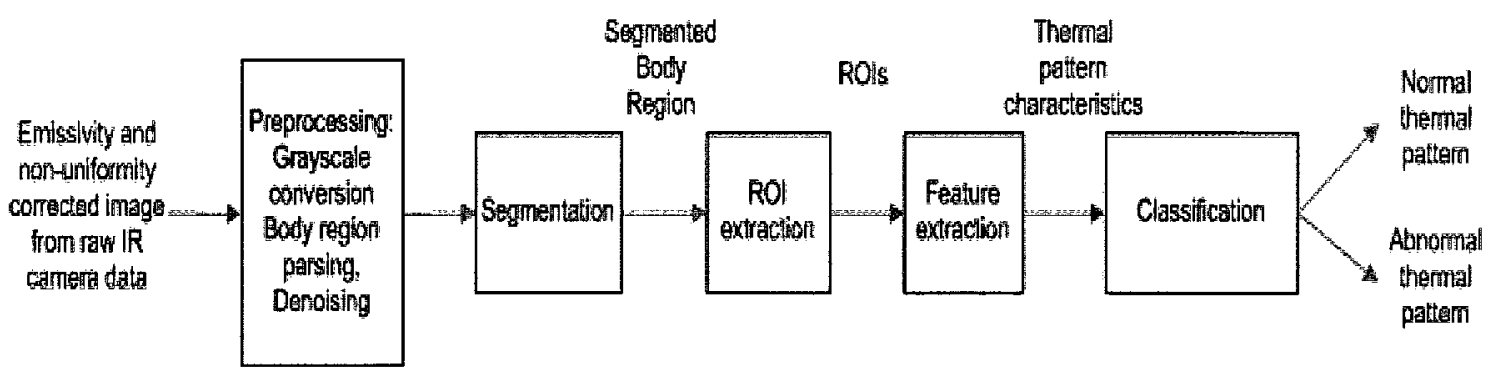

Figure 5-1: Block Diagram of Christophe Herry's Thesis Research (2008) 
Second, to improve the efficiency of the thermal IR diagnostic tool, IR imaging will be combined with multi-spectral imaging to increase its sensitivity and specificity of diagnosis. Multi-spectral imaging would take a sequence of videos with visual cameras to assess the range of motion and level of mobility of the extremities and joints in patients with RA. This would combine two important diagnostic parameters: the distribution and state of the inflamed joints (via IR imaging) and the limitation of motion of the joints (via multi-spectral imaging). This combination of imaging tools would be a highly innovative and promising tool that would be a reliable, quantitative and reproducible means of assessing RA. The ultimate goal is to combine thermal IR technology and multi-spectral imaging and applying it to clinical settings to become a diagnosis-aid that is safe, efficient, inexpensive and easy to operate, and that can be used in health care settings worldwide.

This thesis makes the thermal infrared imaging tool even more a reality as a possible inexpensive, non-invasive and efficient method to diagnose RA. There is more to be explored with thermal infrared technologies; but thus far the results are very promising. One day thermal infrared systems can have a large impact on the quality of life of RA patients, on the Canadian Health Care System and on society as a whole. 


\section{BIBLIOGRAPHY}

American College of Rheumatology Subcommittee on Rheumatoid Arthritis Guidelines. "Guidelines for the management of rheumatoid arthritis 2002 update." Arthritis \& Rheumatism. 46(2): 328-46, 2002.

Anbar, M. "Objective assessment of clinical computerized thermal images." In Medical imaging V: image processing. Vol. 1445 of Proceedings of SPIE, pages 479-484. SPIE-The International Society for Optical Engineers, 1991.

Anbar, M. "Quantitative dynamic telethermometry in medical diagnosis and management." Boca Raton: CRC Press, 1994.

Arnett, F. C. et al. "The American Rheumatism Association 1987 Revised Criteria for the Classification of Rheumatoid Arthritis." Arthritis Rheum 31: 315-24, 1998.

Aubry-Frize, M., G.R.C. Quartey, H. Evans, and D. LaPalme. "The thermographic detection of pain." In Proceedings of the 3rd Canadian Clinical Engineering Conference. pages 82-83, Saskatoon, September 1981.

Bacon, P.A. et al. "Thermography in the assessment of inflammatory arthritis." Clin Rheum Dis. 2:51-65, 1976.

Bednar, Michael S. and Terry R Light. "Chapter 10. Hand Surgery." CURRENT Diagnosis \& Treatment in Orthopedics, 4th Edition. 2003. Accessed on June 15th 2009 <http://www.accessmedicine.com/content.aspx?alD=2314974>.

Bird, H.A., E.F.J. Ring and P.A. Bacon. "A thermographic and clinical comparison of three intra-articular steroid preparations in rheumatoid arthritis." Annals of the Rheumatic Diseases. 28:36-39, 1979.

Bohndorf, K. and Schalm J. "Diagnostic radiography in rheumatoid arthritis: benefits and limitations." Baillière's Clinical Rheumatology. 10: 399-407, 1996.

Brooks, P.M. "MJA Practice Essentials" Rheumatology. Sydney: Australian Medical Publishing Company Limited, 1997.

Bykerk, Vivian P. et al. "Canadian Consensus Statement on Early Optimal Therapy in Early Rheumatoid Arthritis." The Journal of the Canadian 
Rheumatology Association. 2009. Accessed on June 15th 2009 <http://www.rheum.ca/Resources/Pdf/ERA.pdf>.

Collins, A.J and J.A. Cosh. "Temperature and biochemical studies of joint inflammation." Ann. Rheum. Dis. 29: 386, 1970.

Collins, A.J., E.F.J. Ring, J.A. Cosh, and P.A. Bacon. "Quantitation of thermography in arthritis using multiisothermal analysis." Annals of Rheumatoid Diseases. 33:113-115, 1974.

De Silva, M. et al. "Assessment of inflammation in the rheumatoid knee joint: correlation between clinical, radioisotopic, and thermographic methods." Annals of the Theumatic Diesases. 45: 277-280, 1986.

Devereaux, M. et al. "Disease activity indexes in rheumatoid arthritis; a prospective, comparative study with thermography." Annals of the Rheumatic Diseases. 44: 434-437, 1985.

El-Gabalawy, Hani et al. "Chapter 3. Immune Mechanims." Mechanisms in Rheumatology. CD-ROM. Core Health Services Inc., 2001.

Ellis, W.V., J.M. Morris, and A.A. Swartz. "Screening thermography of chronic back pain patients with negative neuromusculoskeletal findings." Thermology. 3, 1989.

Ernisse, B. et al. "Complete automatic target cuer/recognition system for tactical forward-looking infrared images." Optical Engineering. 36: 2593-2603, September 1997.

FLIR Systems. ThermaCAM Researcher User's manual. Sweeden: Publ. No. 1 558071 Rev. a52, June 2004.

M. Frize, J. Karsh, P. Payeur, C. Herry. C. Adea, I Aleem. "Preliminary Results of Severity of Illness Measures of Rheumatoid Arthritis Using Infrared Imaging." Presented at the IEEE International Workshop on Medical Measuremetns and Applications MeMeA. Cetraro, Italie, May 2009.

Gaussorgues, G. "Infrared thermography." Chapman and Hall, 1994.

Gautherie, M., A. Kotewicz, and P Gueblez. "Accurate and objective evaluation of breast thermograms: principles and new advances with special reference to an improved computer-assisted scoring system." Proceedings of the international Conference on Thermal Assessment of Breast Health. Washington, DC, USA. pages 74-92,1983. 
Goldberg, G.S. "Infrared imaging and magnetic resonance imaging correlated in 35 cases." Thermology. 1(4), 1986.

Government of Ontario. "Wait times in Ontario." Ministry of Health and Long Term Care. June $16^{\text {th }}$ 2009. Accessed on June $24^{\text {th }} 2009$ $<$ http://www.health.gov.on.ca/transformation/wait_times/providers/wt_pro_ mn.html>

Goodman, P.H., M.G. Murphy, G.L. Siltanen, M.P. Kelley, and L. Rucker. "Normal temperature asymmetry of the back and extremities by computerassisted infrared imaging." Thermology. 1:195-202, 1986.

Guedes, C. et al. "Mortality in rheumatoid arthritis." Rev Rheum Engl. 66(10): 492-498, 1999.

Haraoui, Boulos, and Edward Keystone. "Chapter 5. Rheumatoid Arthritis." Mechanisms in Rheumatology. CD-ROM. Core Health Services Inc., 2001.

Head, J.F., C.A. Lipari, F. Wang, and R.L. Elliott. "Image analysis of digitized infrared images of the breasts from a first generation infrared imaging system." In Proceedings of the 19th Annual International Conference of the IEEE Engineering in Medicine and Biology Society. pages 681-684, 1997.

Herry, C.L. and M. Frize. "Quantitative assessment of pain-related thermal dysfunction through clinical digital infra-red thermal imaging." Biomedical Engineering, 3:19, 2004.

Herry, C.L., R.A. Goubran and M. Frize. "Segmentation of infrared images using cued morphological processing of edge maps." In Proc. IEEE Instr. Meas. Tech. Conf. Warsaw, May 2007.

Herry, C.L., M. Frize and R.A. Goubran. "Search for abnormal thermal patterns in clinical thermal infrared imaging." In Proceedings of the IEEE International Workshop on Medical Measurements and Applications MeMeA. Ottawa, Ontario, Canada, May 2008.

Herry, C.L., R.A. Goubran, and M. Frize. "Improving the Detection of Localization of Anatomical Landmark Points in Infrared Images Using Summetry and Region Specific Constraints." Proceedings of the $25^{\text {th }}$ IEEE International Instrumentation and Measurement Technology Conference (I2MTC08). Victoria, BC, May 2008. 
Hochberg, M. C. et al. "The American College of Rheumatology 1991 revised criteria for the classification of global functional status in rheumatoid arthritis." Arthritis \& Rheumatism. 35(5):498-502, 1992.

Hooshmand, H., M. Hashmi, and E.M. Phillips. "Infrared thermal imaging as a tool in pain management - an 11 year study, part I of II." Thermology International. 11(2):53-65, 2001.

Horvath, S.M. and J.L. Hollander. "Intra-articular temperature as a measure of joint reaction." J Clinical Invest. 28: 469-73, 1949.

Houdas, Y. and E.F.J. Ring. "Human body temperature: its measurement and regulation." Plenum Press, 1982.

Huang, S.-Y. et al. "A vq-based approach to thermal image analysis for printed circuit boards diagnosis." IEEE Trans. Instrum. Meas. 54:3281-3288, December 2005.

Hubbard, J.E. and C. Hoyt. "Pain evaluation by electronic infrared thermography: correlation with symptoms, emg, myelogram and ct scan." Thermology. 1(1):26-35, 1985.

Hudson Jr., R.D. "Infrared system engineering." Wiley-Interscience. 1969.

llowite, N.T. et al. "Assessment of pain in patients with juvenile rheumatoid arthritis: relation between pain intensity and degree of joint inflammation." Annals of the Rheumatic Diseases. 51: 343-346, 1992.

Irvine, J.M. "Targeting breast cancer detection with military technology." IEEE Engineering in Medicine and Biology Magazine. 21:31-40, 2002.

Jones, B.F. "A reappraisal of the use of infrared thermal image analysis in medicine." IEEE Transactions on Medical Imaging. 17(6):1019-1027, 1998.

Karsh, J. Rheumatologist at the Ottawa Hospital - Riverside Campus. Personal Communication. July $23^{\text {rd }} 2009$.

Karsh, J. Rheumatologist at the Ottawa Hospital - Riverside Campus. Personal Communication. September $22^{\text {nd }} 2009$.

Kim, Y.-S. and Y.-E. Cho. "Correlation of pain severity with thermography." In Proceedings of the 17th Annual International Conference of the IEEE Engineering in Medicine and Biology Society and 21st Canadian Medical 
and Biological Engineering Conference. Volume 2, pages 1699-1700, 1995.

LeRoy, P.L., C.R. Christian, and R. Filasky. "Diagnostic thermography in low back pain syndromes." The Clinical Journal of Pain. 1(1):4-13, 1985.

Lipari, C.A. and J.F. Head. "Advanced infrared image processing for the breast cancer risk assessment." In Proceedings of the 19th Annual International Conference of the IEEE Engineering in Medicine and Biology Society. pages 673-676, 1997.

Lipsky, Peter E., et al. "Chapter 314. Rheumatoid Arthritis." Harrison's Principles of Internal Medicine, 17th Edition. 2008. Accessed on June 15th 2009 <http://www.accessmedicine.com/content.aspx?alD=2859081>.

Matlab R2009a. Software - help files. Version 7.8.0.347, Mathworks 2009.

Moore, Keith L. and Arthur F. Dalley II. Anatomie Médicale - Aspects fondamentaux et applications cliniques, $2^{\text {ieme }}$ édition. Bruxelles : de Boeck, 2007.

Ng, E.Y.K., Y. Chen, and L.N. Ung. "Computerized breast thermography. Study of image segmentation and temperature cyclic variations." Journal of Medical Engineering and Technology. 25:12-16, 2001.

Nielsson, S.K., S.E. Gustafsson, L.M. Torell. "Skin temperature over a heat source: expirmental studies and theoretical calculations. Thermal characteristics of tumours. Applications in detection and treatment." Ann NY Acad. Sci. 335: 516-528, 1980.

O'Dell, James R. et al. "Chapter 15. Rheumatoid Arthritis: The DiseaseDiagnosis and Clinical Features." CURRENT Rheumatology Diagnosis \& Treatment, 2e. 2008. Accessed on June 15th 2009 <http://www.accessmedicine.com/content.aspx?alD=2725004>.

O'Dell, James R. et al. "Chapter 16. Treatment of Rheumatoid Arthritis." CURRENT Rheumatology Diagnosis \& Treatment, 2e. 2008. Accessed on June 15th 2009

<http://www.accessmedicine.com/content.aspx?alD=2725074>.

Poussart, Y., M. Frize, and R. Roberge. "The re-evaluation of thermography in breast cancer detection by new image enhancement techniques." Technical report. Terry Fox Foundation, Fredericton, NB, 1988. 
Reinberg, A. "Circadian changes in the temperature of human beings." Clin. Rheum. Dis. 2:128-39, 1976.

Ring, E.F.J and A.J. Collins. "Quantitative thermography." Rheum Phys Med. 10:337, 1970.

Ring, E.F.J, A.J. Collins, P.A. Bacon, and J.A. Cosh. "Quantitation of thermography in arthritis using multi-isothermal analysis II." Ann. Rheum. Dis. 33:353, 1974.

Ring, E.F.J. "Criteria for thermal imaging in medicine." In IEEE 17th Annual Conference of the Engineering in Medicine and Biology Society. pages 1697-1698, 1995.

Salisbury, R.S. et al. "Heat distribution over normal and abnormal joints: thermal pattern and quantification." Annals of the Rheumatic Diseases. 42: 494499, 1983.

Salvatore, D. and D. Reagle. "Theory and Problems of Statistics and Econometrics - Second Edition." Schaum's Outline Series of McGraw-Hill Companies, 2002.

Scales, N., C.Herry and M. Frize. "Automated Image Segmentation for Breast Analysis Using Infrared Images." In Proceedings EMBS Conf., San Francisco, pages 1737-1740, Sept 2004.

Stolwijk, J. "Medical thermography: theory and clinical applications." Los Angeles: Brentwood Publishing. pages 40-51, 1976.

Sugimoto, H. A. Takeda and K. Hyodoh. "Early-stage rheumatoid arthritis: prospective study of the effectiveness of MR imaging for diagnosis." Radiology. 216: 569-575, 2000.

Symmons, Deborah, Colin Mathers and Bruce Pfleger. "The global burden of rheumatoid arthritis in the year 2000." The global burden of disease. August $15^{\text {th }} 2006$. Accessed on July $2^{\text {nd }} 2009$ $<$ http://www.who.int/healthinfo/statistics/bod_rheumatoidarthritis.pdf $>$.

Thomas, D., D. Cullum, G. Siahamis, and S. Langlois. "Infrared thermographic imaging, magnetic resonance imaging, ct scan and myelography in low back pain." British Journal of Rheumatology. 29:268-273, 1990.

Uematsu, S. "Symmetry of skin temperature comparing one side of the body to the other." Thermology. 1:1, 1985. 
Uematsu, S., D.H. Edwin, W.R. Jankel, J. Kozikowski, and M. Trattner. "Quantification of thermal asymmetry, part 1: Normal values and reproducibility." Journal of Neurosurgery. 69:552-555, 1988.

Wolfe, W. L. "Handbook of military infrared technology." Office of Naval Research, Department of Navy. 1965. 


\section{APPENDIX A}

\section{Permission Text for Potential Participants}

Title: Severity of illness measures in rheumatoid arthritis from multi-spectral imaging

I agree that my name and contact information be released to the researchers of the rheumatoid arthritis imaging research project: Dr. Monique Frize, Dr. Pierre Payeur, and their graduate students: Cynthia Lecavalier and Daphne Townsend.

Name (please print):

Signature:

Contact information:

Address:

Email address:

Telephone numbers: Home

Office

Fax

Cellular

You may contact the student co-investigator Cynthia Lecavalier at (613) 5202600 ext 5586 or email: clecaval@connect.carleton.ca. 


\section{APPENDIX B}

\section{Research Participant Information and Consent Form}

You are being asked to participate in a research study using an infrared (IR) camera (heat images) to determine temperature measurements of limbs. In addition, a series of color pictures using a digital camera will be taken to assess the level of mobility of extremities and joints. The research will be conducted at the University of Ottawa and will be scheduled at a convenient time.

The information collected will not contain pictures of the face and will be kept anonymous.

\section{Purpose and Design}

Rheumatoid arthritis affects 300,000 Canadians each year; it involves inflammation and symptoms like redness, swelling, heat, and pain. In this study, we plan to measure the temperature of the joints of patients diagnosed with rheumatoid arthritis and of participants without rheumatoid arthritis using an infrared camera. The assumption is that the infrared pictures of the rheumatoid arthritis patients will show inflammation of tissue or joints. A second test to be

conducted at the same time is the taking of video pictures with digital cameras to assess the extent of movement of extremities and joints of both groups: patients with rheumatoid arthritis and healthy participants. This is a pilot project to assess whether these two types of tests can be helpful as an aid to the early diagnosis of rheumatoid arthritis. These tests may also help to monitor therapy in joint debilitating diseases.

Approximately 15 patients will be involved in this pilot study and 20 to 30 normal subjects.

Because the skin surface temperature variations are extremely subtle and sensitive to environmental changes:

- Do not use talcum powder, lotion, drug or deodorant on the skin on the day of the session.

- Do not consume alcoholic beverages 12 hours prior to the session.

- Do not consume hot beverages at least 1 hour prior to the session.

- Do not use procedures such as acupuncture, transcutaneous electrical stimulation (TENS machine), hot or cold patches, or any other form of physiotherapy at least $24 \mathrm{~h}$ prior to the session. You should not do any intense physical exercises at least 4 hours prior to the session

- Avoid prolonged sun exposure for at least one week prior to the session.

- Do not smoke at least 2 hours prior to the session.

- Do not wear any rings, necklaces, and bracelets during the session.

\section{- Page 1 of $4-$}


The session will take place in a room with the following characteristics:

- Without windows or with covered windows to avoid any reflection.

- Constant uniform temperature between 20 and 25.

- Constant humidity.

- Fluorescent lights.

You will be asked to come to the biomedical laboratory of Dr. Monique Frize at the University of Ottawa (Colonel By Building, Room C507) at a mutually convenient time. Both types of cameras will be available at that location. You need to wear shorts and short sleeve shirt (or change into these in a nearby bathroom).

The skin has to cool at room temperature for 10-15 minutes; then pictures are taken of the joints, hands, feet. For each body region, you will be asked to assume a position facilitating the imaging. For instance, for images of the hands (palm and back), you will be seated on a chair and will be asked to place your hand over a table. For the under-feet view, you will be seated on a chair and raise your leg, with the ankle resting on a stool. For other views, you will be standing.

You will also be asked to move your hands, elbows, knees and feet from time to time. Sequences of images will be taken with an infrared camera and several regular intraoptical video cameras. Both types of cameras will record the sequences simultaneously.

The imaging session is expected to last approximately 30 minutes.

You may be asked to participate for a maximum of three sessions in order to study the stability of the temperature distribution over time. But this is not mandatory to participate in the study. It is totally voluntary.

\section{Are there any risks?}

There are no foreseen risks to participants.

If you are a patient diagnosed with rheumatoid arthritis, the research team will capture images of your body parts affected by rheumatoid arthritis. If you are a healthy volunteer, the research team will capture images of several healthy joints and limbs.

There is no direct contact or risk associated with gathering these images. 
Are there any benefits to me if I choose to participate? While there are no immediate benefits for you, this study is expected to provide a useful tool for early diagnosis of rheumatoid arthritis.

\section{How will my confidentiality be protected?}

Your name will not be used in any publications that may arise from this study. Only group information will be published. No images of the participant's faces will be taken. The consent forms and images will be stored at Carleton and Ottawa Universities research laboratories of Dr Frize and Dr Payeur containing only numbers as reference. The images will be stored in a protected file with user id and password protection. The data will be destroyed five years after the tests are completed.

\section{Withdrawal from the Study}

If you are a patient with rheumatoid arthritis, you may withdraw from the study at any time without any impact to the care you are receiving. You can also choose to have your images withdrawn from the study completely.

If you are a healthy participant, you may withdraw from the study at any time. You can also choose to have your images withdrawn from the study completely.

\section{Study Expenses}

If you are a patient, you will be reimbursed for parking or bus expenses to attend the session at the University of Ottawa. If you are a student, no compensation will be needed, as you will be on either campus and there is a free bus service between the two universities.

\section{Questions about the Study}

You may ask any questions about the research at any time. If you have questions about the research after you leave today you should contact the student co-investigator Cynthia Lecavalier at (613) 520-2600 ext 5586 or email: clecaval@connect.carleton.ca.

If you have any questions about your rights as a research subject, you may contact the Chairperson of the Ottawa Hospital Research Ethics Board at (613) 798-5555, extension 14902 or the University of Ottawa's Research Ethics Board through the Research Grants and Ethics Services at (613) 562-5841, e-mail ethics@uOttawa.ca. 


\section{Consent}

I have read this Research Participant Information and Consent Form and have had an opportunity to ask my study doctor and the researchers any questions I

had about the study.

My questions and/or concerns have been answered to my satisfaction and I agree to participate in this study. If I decide at a later stage in the study that I would like to withdraw my consent, I may do so at any time.

A copy of the Research Participant Information and Consent Form will be provided to me.

\section{Signatures}

Name of Participant (please print):

Signature

Date

Student co-investigator: Cynthia Lecavalier

Signature

Date 


\section{APPENDIX C}

\section{ThermoVision A40M Researcher}

IMAGING PERFORMANCE

Field of view/min focus distance $24^{\circ} \times 18^{\circ} /$ $0.3 \mathrm{~m}$

Spatial resolution (IFOV) $1.3 \mathrm{mrad}$

Thermal sensitivity @ $50 / 60 \mathrm{~Hz} 0.08^{\circ} \mathrm{C}$ at 30 ${ }^{\circ} \mathrm{C}$

Focusing Built-in focus motor

Detector type Focal Plane Array (FPA), uncooled microbolometer

Spectral range 7.5 to $13 \mu \mathrm{m}$

IMAGE PRESENTATION

FireWire output IEEE-1394 8/16-bit monochrome and 8-bit color

Video output RS170 EIAJNTSC or CCIR/PAL composite video

\section{MEASUREMENT}

\section{Temperature ranges}

Range 1: $-40^{\circ} \mathrm{C}$ to $+120^{\circ} \mathrm{C} \quad(-40$ to $+248^{\circ} \mathrm{F}$ )

Range 2: $0^{\circ} \mathrm{C}$ to $+500^{\circ} \mathrm{C} \quad(+32$ to $+932^{\circ} \mathrm{F}$ )

Optional: Up to $+1500^{\circ} \mathrm{C}\left(+2732^{\circ} \mathrm{F}\right)$

Optional: Up to $+2000^{\circ} \mathrm{C}\left(+3632^{\circ} \mathrm{F}\right)$

Accuracy (\% of reading) $\pm 2{ }^{\circ} \mathrm{C}$ or $\pm 2 \%$

Measurement modes Spot, Area, Isotherm, Difference

Automatic emissivity Variable from 0.1 to 1.0

Individual emissivity settings Individually settable

Measurement corrections Reflected ambient, distance, relative humidity, external optics. Automatic, based on user input

\section{POWER SOURCE}

AC operation AC adapter 110/220 VAC, $50 / 60 \mathrm{~Hz}$ (included)

DC operation 8-30V nominal, $<6 \mathrm{~W}$

\section{PHYSICAL CHARACTERISTICS}

Weight $1.4 \mathrm{~kg}$ (3.0 lbs)

Size $207 \mathrm{~mm} \times 92 \mathrm{~mm} \times 109 \mathrm{~mm}\left(8.1^{\prime \prime} \times 3.6^{\prime \prime} \times\right.$ 4.3")

Tripod mounting 1/4"- 20

\section{ENVIRONMENTAL}

Operating temperature range $-15^{\circ} \mathrm{C}$ to $+50^{\circ} \mathrm{C}$ $\left(5^{\circ} \mathrm{F}\right.$ to $\left.122^{\circ} \mathrm{F}\right)$

Storage temperature range $-40^{\circ} \mathrm{C}$ to $+70^{\circ} \mathrm{C}(-$ $40^{\circ} \mathrm{F}$ to $158^{\circ} \mathrm{F}$ )

Humidity Operating and storage $10 \%$ to $95 \%$, non-condensing

Encapsulation

IP

40

(Determined by connector type)

Shock Operational: 25G, IEC 68-2-29

Vibration Operational: 2G, IEC 68-2-6 


\title{
10 APPENDIX D
}

\author{
$10.12^{\text {nd }}$ MCP Raw Data Measurements
}

Table 10-1: Raw Data Measurements Extracted from the Control Group's 2nd MCP Joint

\begin{tabular}{|c|c|c|c|c|c|c|c|c|c|}
\hline & & $\operatorname{Max}\left({ }^{\circ} \mathrm{C}\right)$ & $\operatorname{Min}\left({ }^{\circ} \mathrm{C}\right)$ & Mean $\left({ }^{\circ} \mathrm{C}\right)$ & Median ( ${ }^{\circ} \mathrm{C}$ ) & Mode $\left({ }^{\circ} \mathrm{C}\right)$ & STD & Skewness & Kurtosis \\
\hline \multirow{18}{*}{ 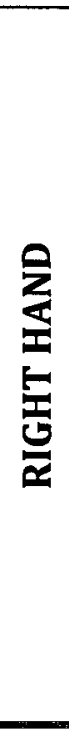 } & 1 & 26,1105 & 24,6853 & 25,4597 & 25,5042 & 25,4580 & 0,3534 & $-0,2794$ & 1,9844 \\
\hline & 2 & 28,3482 & 25,7030 & 27,1593 & 27,1930 & 27,2386 & 0,6124 & $-0,1033$ & 2,1580 \\
\hline & 3 & 27,8476 & 26,9645 & 27,3119 & 27,3050 & 27,2370 & 0,1473 & 0,1713 & 2,7103 \\
\hline & 4 & 30,0950 & 28,8796 & 29,2699 & 29,1995 & 29,1624 & 0,2359 & 1,1101 & 3,6497 \\
\hline & 5 & 31,9448 & 30,4923 & 31,0995 & 31,0931 & 30,8663 & 0,3064 & 0,1588 & 2,2105 \\
\hline & 6 & 34,4558 & 33,0637 & 33,6921 & 33,6296 & 33,2861 & 0,3598 & 0,2760 & 1,9135 \\
\hline & 7 & 31,9741 & 30,4487 & 30,9595 & 30,8778 & 30,7716 & 0,3280 & 0,5527 & 2,2921 \\
\hline & 8 & 32,8184 & 30,8655 & 31,4970 & 31,4279 & 31,2164 & 0,3612 & 0,6998 & 2,8610 \\
\hline & 9 & $x$ & $x$ & $\mathrm{x}$ & $\mathbf{x}$ & $x$ & $x$ & $x$ & $x$ \\
\hline & 10 & 32,9767 & 31,8570 & 32,3339 & 32,3496 & 32,4796 & 0,2265 & 0,0531 & 2,6858 \\
\hline & 11 & 27,5611 & 25,9822 & 26,4582 & 26,3486 & 26,2190 & 0,2976 & 0,9900 & 3,1782 \\
\hline & 12 & 27,3503 & 25,4305 & 26,5945 & 26,6153 & 26,9872 & 0,4126 & $-0,3342$ & 2,5027 \\
\hline & 13 & 24,4499 & 22,1455 & 23,2691 & 23,2255 & 23,0450 & 0,4804 & 0,2267 & 2,3356 \\
\hline & 14 & 26,7441 & 24,3879 & 25,5904 & 25,6069 & 25,6069 & 0,4945 & $-0,0251$ & 2,4962 \\
\hline & 15 & 33,3864 & 32,3649 & 32,8731 & 32,8552 & 32,6824 & 0,2387 & 0,0836 & 1,9904 \\
\hline & 16 & 29,2447 & 27,5989 & 28,1753 & 28,1477 & 28,0727 & 0,3244 & 0,4250 & 2,5414 \\
\hline & 17 & 32,5950 & 30,6676 & 31,3209 & 31,2675 & 31,2602 & 0,3478 & 0,7991 & 3,6520 \\
\hline & 18 & 33,0994 & 32,1115 & 32,6186 & 32,6317 & 32,6606 & 0,2271 & $-0,0597$ & 2,0101 \\
\hline \multirow{18}{*}{$\frac{Q}{\sum}$} & 1 & 26,2329 & 24,2346 & 25,1966 & 25,1496 & 24,9796 & 0,3884 & 0,3312 & 2,7780 \\
\hline & 2 & 27,7155 & 25,5182 & 26,6314 & 26,6605 & 26,6605 & 0,5214 & $-0,0739$ & 2,1749 \\
\hline & 3 & 26,6837 & 24,7830 & 25,6857 & 25,6685 & 25,4382 & 0,3921 & 0,2635 & 2,5750 \\
\hline & 4 & 29,2590 & 27,3793 & 28,2671 & 28,2519 & 27,9894 & 0,3828 & 0,1800 & 2,1998 \\
\hline & 5 & 32,2273 & 30,9688 & 31,6088 & 31,6689 & 31,7851 & 0,2859 & $-0,4344$ & 2,1899 \\
\hline & 6 & 34,8808 & 33,1283 & 33,9226 & 33,9366 & 33,2933 & 0,4055 & 0,0045 & 2,0417 \\
\hline & 7 & 32,8696 & 31,5455 & 31,8960 & 31,8363 & 31,7854 & 0,2206 & 1,3763 & 5,4255 \\
\hline & 8 & 32,9048 & 30,4474 & 31,2677 & 31,2748 & 31,1215 & 0,4350 & 0,7281 & 4,1835 \\
\hline & 9 & $\mathrm{x}$ & $x$ & $x$ & $x$ & $x$ & $x$ & $x$ & $x$ \\
\hline & 10 & 33,5432 & 31,7408 & 32,2995 & 32,2556 & 32,1687 & 0,2675 & 1,2854 & 5,7058 \\
\hline & 11 & 26,7669 & 24,6901 & 25,6044 & 25,5494 & 25,5993 & 0,4262 & 0,4738 & 2,7667 \\
\hline & 12 & 26,7445 & 25,0764 & 25,8706 & 25,8524 & 25,8524 & 0,3327 & 0,2228 & 2,4346 \\
\hline & 13 & 24,2248 & 21,6849 & 22,9110 & 22,8877 & 22,7302 & 0,5093 & 0,0442 & 2,6394 \\
\hline & 14 & 26,5998 & 23,6092 & 25,1774 & 25,2419 & 24,7829 & 0,6574 & $-0,1606$ & 2,0324 \\
\hline & 15 & 33,5796 & 32,3577 & 32,8979 & 32,9056 & 32,8696 & 0,2155 & 0,3059 & 3,2486 \\
\hline & 16 & 29,2818 & 27,7119 & 28,3897 & 28,3575 & 28,2377 & 0,3109 & 0,2165 & 2,4786 \\
\hline & 17 & 31,5299 & 29,9982 & 30,7524 & 30,7996 & 30,4327 & 0,3546 & $-0,1281$ & 2,0290 \\
\hline & 18 & 32,9413 & 31,8214 & 32,4148 & 32,4477 & 32,4586 & 0,2620 & $-0,2772$ & 2,1671 \\
\hline \multicolumn{2}{|c|}{ Average } & 29,9702 & 28,2454 & 29,0140 & 29,0007 & 28,8952 & 0,3565 & 0,2677 & 2,7130 \\
\hline
\end{tabular}


Table 10-2: Raw Data Measurements Extracted from the Patient Group's 2nd MCP Joint

\begin{tabular}{|c|c|c|c|c|c|c|c|c|c|}
\hline & & $\operatorname{Max}\left({ }^{\circ} \mathrm{C}\right)$ & $\operatorname{Min}\left({ }^{\circ} \mathrm{C}\right)$ & Mean $\left({ }^{\circ} \mathrm{C}\right)$ & $\begin{array}{l}\text { Median } \\
\left({ }^{\circ} \mathrm{C}\right)\end{array}$ & Mode $\left({ }^{\circ} \mathrm{C}\right)$ & STD & Skewness & Kurtosis \\
\hline \multirow{13}{*}{ 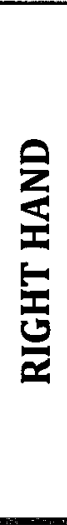 } & 1 & 34,1853 & 32,5157 & 32,9144 & 32,8760 & 32,7752 & 0,2123 & 1,8015 & 8,9345 \\
\hline & 2 & 34,3468 & 32,9070 & 33,5189 & 33,5539 & 33,7114 & 0,2631 & $-0,0834$ & 2,7726 \\
\hline & 3 & 33,6470 & 31,7626 & 32,7416 & 32,8710 & 33,0655 & 0,4647 & $-0,2646$ & 1,6601 \\
\hline & 4 & 30,0216 & 28,3053 & 29,0348 & 29,0589 & 29,3783 & 0,3849 & $-0,0057$ & 1,8287 \\
\hline & 5 & 29,8801 & 28,3182 & 29,2812 & 29,3025 & 29,2802 & 0,3420 & $-0,4080$ & 2,4527 \\
\hline & 6 & 31,6099 & 27,4611 & 29,1036 & 28,9679 & 28,8114 & 0,9284 & 0,4308 & 2,3475 \\
\hline & 7 & 34,1497 & 32,4363 & 33,2885 & 33,2890 & 33,2639 & 0,2014 & $-0,0640$ & 3,8788 \\
\hline & 8 & 32,9120 & 31,7341 & 32,2329 & 32,1835 & 32,0749 & 0,2439 & 0,4930 & 2,4673 \\
\hline & 9 & 31,2683 & 29,5413 & 30,2286 & 30,2426 & 30,1911 & 0,2889 & 0,0198 & 2,8278 \\
\hline & 10 & 33,9721 & 31,4287 & 32,4823 & 32,4658 & 32,7470 & 0,5197 & 0,2233 & 2,3592 \\
\hline & 11 & 33,7791 & 31,7989 & 32,5586 & 32,5878 & 32,8184 & 0,3941 & $-0,0181$ & 1,9991 \\
\hline & 12 & 32,6211 & 31,3234 & 31,8121 & 31,8036 & 31,8254 & 0,2546 & 0,3638 & 2,7489 \\
\hline & 13 & 28,5139 & 27,5378 & 28,0228 & 28,0569 & 28,1170 & 0,1676 & $-0,5022$ & 2,8177 \\
\hline \multirow{13}{*}{$\underset{1}{2}$} & 1 & 33,3571 & 32,3134 & 32,7815 & 32,7824 & 32,7176 & 0,1961 & $-0,0138$ & 2,5599 \\
\hline & 2 & 33,7114 & 31,8063 & 33,2150 & 33,2453 & 33,5610 & 0,2907 & $-0,9527$ & 4,4021 \\
\hline & 3 & 34,1972 & 27,4902 & 33,4464 & 33,4965 & 33,6399 & 0,4492 & $-5,7915$ & 57,5579 \\
\hline & 4 & 30,1469 & 28,3801 & 29,5020 & 29,5117 & 29,5117 & 0,2609 & $-0,3176$ & 3,5379 \\
\hline & 5 & 29,3841 & 27,5893 & 28,5867 & 28,5875 & 28,4455 & 0,3419 & $-0,0962$ & 2,3800 \\
\hline & 6 & 28,5128 & 26,3002 & 27,2766 & 27,2760 & 26,9391 & 0,4515 & 0,1988 & 2,4525 \\
\hline & 7 & 33,6004 & 32,1542 & 33,0773 & 33,1060 & 33,0126 & 0,2997 & $-0,7272$ & 3,1715 \\
\hline & 8 & 33,0844 & 31,5378 & 32,3608 & 32,4148 & 32,5808 & 0,3553 & $-0,1136$ & 1,9768 \\
\hline & 9 & 31,7268 & 30,2058 & 30,9235 & 30,9103 & 31,3266 & 0,3486 & $-0,0327$ & 1,9586 \\
\hline & 10 & 33,8295 & 31,6325 & 32,6355 & 32,6101 & 32,4658 & 0,5274 & 0,1053 & 2,0062 \\
\hline & 11 & 33,5289 & 32,1325 & 32,9158 & 32,9982 & 33,0342 & 0,3331 & $-0,4642$ & 2,2452 \\
\hline & 12 & 32,4913 & 31,4327 & 31,9641 & 31,9813 & 32,0502 & 0,2404 & $-0,0672$ & 2,1993 \\
\hline & 13 & 29,1401 & 28,1920 & 28,6156 & 28,5737 & 28,4391 & 0,1941 & 0,3605 & 2,3810 \\
\hline \multicolumn{2}{|c|}{ Average } & 32,2161 & 30,3168 & 31,3277 & 31,3367 & 31,3763 & 0,3444 & $-0,2279$ & 4,9201 \\
\hline
\end{tabular}


10.2 $3^{\text {rd }}$ MCP Raw Data Measurements

Table 10-3: Raw Data Measurements Extracted from the Control Group's 3rd MCP Joint

\begin{tabular}{|c|c|c|c|c|c|c|c|c|c|}
\hline & & $\operatorname{Max}\left({ }^{\circ} \mathrm{C}\right)$ & $\operatorname{Min}\left({ }^{\circ} \mathrm{C}\right)$ & $\begin{array}{l}\text { Mean } \\
\left({ }^{\circ} \mathrm{C}\right)\end{array}$ & $\begin{array}{c}\text { Median } \\
\left({ }^{\circ} \mathrm{C}\right)\end{array}$ & Mode $\left({ }^{\circ} \mathrm{C}\right)$ & STD & Skewness & Kurtosis \\
\hline \multirow{18}{*}{ 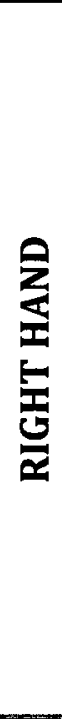 } & 1 & 25,9880 & 24,4990 & 25,2549 & 25,2653 & 24,9099 & 0,3747 & 0,0264 & 2,0235 \\
\hline & 2 & 28,8054 & 27,2082 & 28,1365 & 28,1678 & 28,4833 & 0,3883 & $-0,2086$ & 1,9324 \\
\hline & 3 & 28,1704 & 27,0402 & 27,6344 & 27,6595 & 27,7423 & 0,2372 & $-0,2217$ & 2,2984 \\
\hline & 4 & 30,3895 & 29,2887 & 29,7749 & 29,7406 & 29,6297 & 0,2470 & 0,3682 & 2,2880 \\
\hline & 5 & 31,6907 & 30,3086 & 30,8597 & 30,8224 & 30,6758 & 0,3011 & 0,4344 & 2,3225 \\
\hline & 6 & 34,3919 & 32,4155 & 33,1733 & 33,0996 & 33,0206 & 0,4710 & 0,5141 & 2,5070 \\
\hline & 7 & 32,3649 & 30,7349 & 31,1827 & 31,1300 & 30,9985 & 0,2778 & 1,0317 & 4,2677 \\
\hline & 8 & 31,9513 & 30,6896 & 31,1362 & 31,1361 & 30,8435 & 0,2498 & 0,4963 & 2,7302 \\
\hline & 9 & $x$ & $x$ & $x$ & $x$ & $x$ & $x$ & $x$ & $x$ \\
\hline & 10 & 32,5734 & 31,5226 & 31,9505 & 31,9441 & 32,0746 & 0,2118 & 0,2967 & 2,5494 \\
\hline & 11 & 27,5762 & 26,8807 & 27,1890 & 27,1761 & 27,0172 & 0,1694 & 0,1757 & 2,0087 \\
\hline & 12 & 27,8551 & 26,3108 & 27,1695 & 27,1765 & 27,1538 & 0,2794 & $-0,3697$ & 3,2960 \\
\hline & 13 & 24,4112 & 22,8169 & 23,6351 & 23,6326 & 23,4762 & 0,3256 & $-0,0919$ & 2,3508 \\
\hline & 14 & 27,3121 & 25,8369 & 26,6115 & 26,6378 & 26,7897 & 0,3297 & $\begin{array}{l}-0,3488 \\
\end{array}$ & 2,3923 \\
\hline & 15 & 32,9127 & 32,2348 & 32,4359 & 32,4372 & 32,4516 & 0,1082 & 0,5938 & 3,7834 \\
\hline & 16 & 28,9249 & 27,2517 & 27,8189 & 27,7495 & 27,6140 & 0,3378 & 0,6234 & 2,6660 \\
\hline & 17 & 32,1180 & 30,5869 & 31,0833 & 31,0338 & 30,8875 & 0,2834 & 0,6766 & 2,9600 \\
\hline & 18 & 33,1855 & 32,1260 & 32,5662 & 32,5380 & 32,3719 & 0,2448 & 0,3700 & 2,2187 \\
\hline \multirow{18}{*}{ 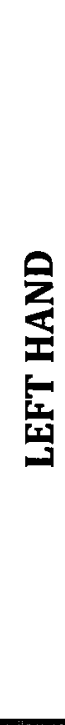 } & 1 & 27,9548 & 26,5154 & 27,2260 & 27,1995 & 27,0858 & 0,3569 & 0,1274 & 2,0021 \\
\hline & 2 & 28,8129 & 27,3523 & 28,1262 & 28,1226 & 27,9042 & 0,3491 & $-0,0138$ & 2,1087 \\
\hline & 3 & 27,5616 & 26,1888 & 26,8446 & 26,8432 & 26,6077 & 0,2789 & 0,0939 & 2,4005 \\
\hline & 4 & 29,6000 & 28,5288 & 29,1702 & 29,1772 & 29,1327 & 0,2202 & $-0,3065$ & 2,3702 \\
\hline & 5 & 32,1622 & 30,6685 & 31,1935 & 31,1442 & 30,7931 & 0,3027 & 0,5650 & 2,6446 \\
\hline & 6 & 34,4487 & 32,5815 & 33,3944 & 33,4007 & 33,6511 & 0,4172 & 0,0447 & 1,9892 \\
\hline & 7 & 32,5310 & 31,3926 & 31,8374 & 31,7600 & 31,5965 & 0,2727 & 0,7486 & 2,4340 \\
\hline & 8 & 31,6390 & 30,2856 & 30,7270 & 30,6750 & 30,5062 & 0,2576 & 0,8266 & 3,2548 \\
\hline & 9 & $\mathrm{x}$ & $\mathrm{x}$ & $x$ & $x$ & $x$ & $x$ & $x$ & $x$ \\
\hline & 10 & 32,7896 & 31,5517 & 32,0198 & 31,9803 & 31,8062 & 0,2510 & 0,4639 & 2,5095 \\
\hline & 11 & 27,4707 & 26,2342 & 26,8916 & 26,9186 & 27,0474 & 0,2874 & $-0,2636$ & 2,1485 \\
\hline & 12 & 28,0504 & 26,6077 & 27,0681 & 27,0213 & 26,9417 & 0,2551 & 0,8292 & 3,6955 \\
\hline & 13 & 24,6359 & 23,2334 & 23,9262 & 23,9291 & 23,7341 & 0,3178 & 0,0527 & 2,1870 \\
\hline & 14 & 27,2970 & 25,9669 & 26,6052 & 26,5960 & 26,3715 & 0,3121 & 0,1191 & 2,0681 \\
\hline & 15 & 33,0924 & 32,3649 & 32,6331 & 32,6175 & 32,5021 & 0,1386 & 0,3769 & 2,5512 \\
\hline & 16 & 29,6376 & 27,8022 & 28,5544 & 28,5221 & 28,2227 & 0,3862 & 0,4670 & 2,6062 \\
\hline & 17 & 31,5445 & 30,1088 & 30,5492 & 30,4988 & 30,3739 & 0,2751 & 0,9564 & 3,6453 \\
\hline & 18 & 33,1066 & 31,7052 & 32,1949 & 32,1658 & 31,9955 & 0,2696 & 0,5151 & 2,7192 \\
\hline \multicolumn{2}{|c|}{ Average } & 30,0869 & 28,7306 & 29,3110 & 29,2917 & 29,1886 & 0,2878 & 0,2932 & 2,5862 \\
\hline
\end{tabular}


Table 10-4: Raw Data Measurements Extracted from the Patient Group's 3rd MCP Joint

\begin{tabular}{|c|c|c|c|c|c|c|c|c|c|}
\hline & & $\operatorname{Max}\left({ }^{\circ} \mathrm{C}\right)$ & $\operatorname{Min}\left({ }^{\circ} \mathrm{C}\right)$ & Mean ( $\left.{ }^{\circ} \mathrm{C}\right)$ & $\begin{array}{c}\text { Median } \\
\left({ }^{\circ} \mathrm{C}\right)\end{array}$ & Mode $\left({ }^{\circ} \mathrm{C}\right)$ & STD & Skewness & Kurtosis \\
\hline \multirow{13}{*}{ 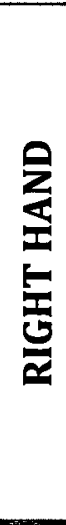 } & 1 & 33,7148 & 32,7104 & 33,0718 & 33,0629 & 32,9479 & 0,1634 & 0,6039 & 3,7875 \\
\hline & 2 & 34,2114 & 33,1590 & 33,6750 & 33,6971 & 33,9117 & 0,2670 & $-0,0658$ & 1,7664 \\
\hline & 3 & 33,4965 & 32,6184 & 33,1865 & 33,1878 & 33,1662 & 0,1218 & $-0,5207$ & 4,0262 \\
\hline & 4 & 30,0953 & 28,8356 & 29,2370 & 29,1853 & 29,1036 & 0,2460 & 1,5795 & 5,5391 \\
\hline & 5 & 29,9465 & 29,0944 & 29,5748 & 29,6065 & 29,6953 & 0,1492 & $-0,4995$ & 2,6517 \\
\hline & 6 & 30,5062 & 29,5176 & 29,8991 & 29,9022 & 29,9392 & 0,2060 & 0,4202 & 2,8930 \\
\hline & 7 & 33,7362 & 32,7968 & 33,3208 & 33,3427 & 33,5504 & 0,2495 & $-0,2523$ & 1,7555 \\
\hline & 8 & 32,7681 & 31,7849 & 32,2695 & 32,2558 & 32,1835 & 0,1719 & 0,0768 & 2,7501 \\
\hline & 9 & 30,8371 & 29,6671 & 30,0733 & 30,0216 & 29,9257 & 0,2342 & 0,7954 & 3,1084 \\
\hline & 10 & 33,4865 & 31,8650 & 32,2666 & 32,1839 & 32,1332 & 0,2519 & 1,7503 & 6,5791 \\
\hline & 11 & 33,3069 & 31,8498 & 32,4927 & 32,4796 & 32,2917 & 0,3363 & 0,2096 & 2,1556 \\
\hline & 12 & 32,6643 & 31,6147 & 32,0417 & 32,0502 & 32,1298 & 0,2147 & 0,1873 & 2,3189 \\
\hline & 13 & 28,5438 & 27,9368 & 28,2591 & 28,2519 & 28,1320 & 0,1193 & 0,2457 & 2,4683 \\
\hline \multirow{13}{*}{$\underset{1}{2}$} & 1 & 33,3284 & 32,5229 & 32,9428 & 32,9407 & 32,9695 & 0,1485 & $-0,0659$ & 2,8655 \\
\hline & 2 & 33,3387 & 32,5822 & 33,0151 & 33,0223 & 32,9863 & 0,1379 & $-0,6092$ & 3,3563 \\
\hline & 3 & 34,0902 & 32,8566 & 33,6180 & 33,6542 & 33,7043 & 0,2345 & $-0,8674$ & 3,7121 \\
\hline & 4 & 30,7639 & 29,3486 & 29,7821 & 29,6967 & 29,6079 & 0,3071 & 0,9006 & 3,0437 \\
\hline & 5 & 29,3915 & 28,6921 & 29,0793 & 29,0833 & 29,0721 & 0,1437 & $-0,2263$ & 2,5309 \\
\hline & 6 & 29,2876 & 27,8077 & 28,4814 & 28,4155 & 28,1533 & 0,3390 & 0,4014 & 2,1294 \\
\hline & 7 & 33,6648 & 32,5373 & 33,1810 & 33,2208 & 33,5074 & 0,2846 & $-0,4192$ & 2,1694 \\
\hline & 8 & 33,0054 & 31,5159 & 32,2548 & 32,2342 & 31,8647 & 0,3351 & 0,2685 & 2,1979 \\
\hline & 9 & 31,7994 & 29,4376 & 30,1800 & 30,1137 & 30,1174 & 0,4380 & 0,8169 & 3,5398 \\
\hline & 10 & 33,4435 & 32,0100 & 32,5267 & 32,4658 & 32,4514 & 0,3063 & 0,6538 & 2,7593 \\
\hline & 11 & 33,4430 & 32,1325 & 32,7466 & 32,6960 & 32,9335 & 0,3346 & 0,2709 & 1,9305 \\
\hline & 12 & 32,7076 & 31,4691 & 31,9673 & 31,9560 & 31,9125 & 0,2450 & 0,2584 & 2,4530 \\
\hline & 13 & 29,4667 & 28,5811 & 28,9966 & 28,9690 & 28,8349 & 0,1869 & 0,3749 & 2,3855 \\
\hline \multicolumn{2}{|c|}{ Average } & 32,1171 & 30,9594 & 31,4669 & 31,4498 & 31,4317 & 0,2374 & 0,2418 & 2,9567 \\
\hline
\end{tabular}




\section{$10.32^{\text {nd }}$ PIP Raw Data Measurements}

Table 10-5: Raw Data Measurements Extracted from the Control Group's 2nd PIP Joint

\begin{tabular}{|c|c|c|c|c|c|c|c|c|c|}
\hline & & $\operatorname{Max}\left({ }^{\circ} \mathrm{C}\right)$ & $\operatorname{Min}\left({ }^{\circ} \mathrm{C}\right)$ & $\begin{array}{c}\text { Mean } \\
\text { ('C) }\end{array}$ & Median ("C) & Mode $\left({ }^{\circ} \mathrm{C}\right)$ & STD & Skewness & Kurtosis \\
\hline \multirow{18}{*}{$\begin{array}{l}2 \\
\frac{1}{5} \\
\frac{5}{5}\end{array}$} & 1 & 24,1645 & 22,9971 & 23,6758 & 23,7037 & 23,8445 & 0,2556 & $-0,3692$ & 2,2885 \\
\hline & 2 & 25,0006 & 23,1654 & 24,1359 & 24,1845 & 24,2547 & 0,4384 & $-0,2647$ & 2,0702 \\
\hline & 3 & 26,7065 & 26,0666 & 26,4829 & 26,5088 & 26.5164 & 0,1115 & $-1,0337$ & 4,0433 \\
\hline & 4 & 30,0065 & 28,8796 & 29,3577 & 29,3184 & 29,1327 & 0,2651 & 0,4385 & 2,2370 \\
\hline & 5 & 32,1622 & 30,8297 & 31,6574 & 31,6398 & 31,5379 & 0,2405 & $-0,1575$ & 2,8235 \\
\hline & 6 & 34,5834 & 33,4723 & 34,1562 & 34,1929 & 34,2924 & 0,2280 & $-0,6434$ & 3,1801 \\
\hline & 7 & 31,9886 & 30,7496 & 31,2844 & 31,2322 & 30,5379 & 0,3231 & 0,2941 & 1,9904 \\
\hline & 8 & 32,8688 & 31,7626 & 32,2721 & 32,2339 & 31,9948 & 0,2631 & 0,2795 & 2,0386 \\
\hline & 9 & $x$ & $x$ & $x$ & $x$ & $x$ & $x$ & $x$ & $x$ \\
\hline & 10 & 33,1850 & 32,2049 & 32,6823 & 32,6527 & 32,5734 & 0,1700 & 0,2976 & 2,8805 \\
\hline & 11 & 27,2819 & 25,7909 & 26,5397 & 26,5237 & 26,4629 & 0,3256 & 0,1131 & 2,3854 \\
\hline & 12 & 25,3306 & 24,1783 & 24,6459 & 24,6205 & 24,4733 & 0,2401 & 0,4108 & 2,3964 \\
\hline & 13 & 20,7094 & 20,0162 & 20,3398 & 20,3473 & 20,3876 & 0,1384 & 0,1624 & 2,3345 \\
\hline & 14 & 23,9914 & 22,4619 & 23,1525 & 23,1784 & 23,2334 & 0,3645 & $-0,0143$ & 2,0142 \\
\hline & 15 & 33,9864 & 32,5310 & 33,4785 & 33,5582 & 33,7368 & 0,3245 & $-0,8970$ & 2,9950 \\
\hline & 16 & 29,9628 & 28,6192 & 29,2112 & 29,2521 & 28,6938 & 0,3328 & 0,1190 & 1,9648 \\
\hline & 17 & 33,1132 & 31,9078 & 32,5747 & 32,5734 & 32,7968 & 0,2797 & $-0,1401$ & 2,1567 \\
\hline & 18 & 32,8550 & 31,6325 & 32,1400 & 32,1187 & 31,9520 & 0,2531 & 0,5517 & 2,9350 \\
\hline \multirow{18}{*}{$\frac{9}{2}$} & 1 & 24,5611 & 23,3588 & 24,2386 & 24,2813 & 24,2968 & 0,1766 & $-1,3103$ & 5,3514 \\
\hline & 2 & 25,1709 & 22,9999 & 24,0704 & 24,0596 & 23,9346 & 0,5772 & 0,0452 & 1,7740 \\
\hline & 3 & 25,0456 & 24,4268 & 24,6976 & 24,6825 & 24,5973 & 0,1102 & 0,2399 & 2,7099 \\
\hline & 4 & 28,0119 & 26,2863 & 26,9189 & 26,8721 & 26,7279 & 0,3410 & 0,7798 & 3,2300 \\
\hline & 5 & 32,4441 & 31,2902 & 31,8711 & 31,8577 & 31,6252 & 0,2771 & 0,0158 & 1,9755 \\
\hline & 6 & 34,8454 & 33,8724 & 34,3417 & 34,3635 & 34,4487 & 0,2062 & $-0,0742$ & 2,4332 \\
\hline & 7 & 32,3216 & 30,6763 & 31,6273 & 31,6328 & 31,6328 & 0,1667 & $-0,3021$ & 8,1294 \\
\hline & 8 & 32,4074 & 30,8875 & 31,6825 & 31,6754 & 31,4061 & 0,3058 & 0,0217 & 2,2089 \\
\hline & 9 & $x$ & $x$ & $x$ & $x$ & $x$ & $x$ & $x$ & $x$ \\
\hline & 10 & 33,1850 & 31,8860 & 32,6281 & 32,6023 & 32,6023 & 0,1756 & 0,4422 & 4,8155 \\
\hline & 11 & 25,9287 & 24,5662 & 25,0524 & 25,0377 & 25,2842 & 0,2772 & 0,4919 & 2,6216 \\
\hline & 12 & 26,3032 & 25,0147 & 25,5711 & 25,5765 & 25,2613 & 0,3050 & 0,2818 & 2,1821 \\
\hline & 13 & 21,2862 & 20,4198 & 20,8147 & 20,7977 & 20,7736 & 0,1853 & 0,1875 & 2,1033 \\
\hline & 14 & 24,0771 & 22,7696 & 23,2827 & 23,2647 & 23,1784 & 0,2372 & 0,4446 & 2,8447 \\
\hline & 15 & 33,4795 & 32,3143 & 33,2097 & 33,2359 & 33,2144 & 0,1746 & $-2,0751$ & 9,1976 \\
\hline & 16 & 29,5043 & 28,1927 & 28,5862 & 28,5295 & 28,4099 & 0,2556 & 1,0043 & 4,0426 \\
\hline & 17 & 32,0746 & 30,8948 & 31,4185 & 31,4206 & 31,5372 & 0,2580 & 0,1484 & 2,3501 \\
\hline & 18 & 33,0060 & 31,7415 & 32,4962 & 32,5524 & 32,6461 & 0,2759 & $-0,6364$ & 2,7538 \\
\hline \multicolumn{2}{|c|}{ Average } & 29,1632 & 27,9077 & 28,5381 & 28,5377 & 28,4705 & 0,2606 & $-0,0338$ & 3,0429 \\
\hline
\end{tabular}


Table 10-6: Raw Data Measurements Extracted from the Patient Group's 2nd PIP Joint

\begin{tabular}{|c|c|c|c|c|c|c|c|c|c|}
\hline & & $\operatorname{Max}\left({ }^{\circ} \mathrm{C}\right)$ & $\operatorname{Min}\left({ }^{\circ} \mathrm{C}\right)$ & $\begin{array}{l}\text { Mean } \\
\left({ }^{\circ} \mathrm{C}\right)\end{array}$ & Median ( ${ }^{\circ} \mathrm{C}$ ) & Mode $\left({ }^{\circ} \mathrm{C}\right)$ & STD & Skewness & Kurtosis \\
\hline \multirow{13}{*}{$\begin{array}{l}\frac{Q}{2} \\
\frac{1}{2} \\
\frac{5}{2}\end{array}$} & 1 & 33,2926 & 32,2483 & 32,9118 & 32,9623 & 33,1060 & 0,2411 & $-0,5396$ & 2,3129 \\
\hline & 2 & 34,1900 & 32,9791 & 33,7927 & 33,8259 & 33,9546 & 0,2213 & $-0,9457$ & 3,8211 \\
\hline & 3 & 34,1330 & 31,9444 & 32,7004 & 32,5895 & 32,3724 & 0,3737 & 0,9882 & 3,5413 \\
\hline & 4 & 29,5561 & 28,5147 & 28,9690 & 28,9548 & 28,9473 & 0,2140 & 0,3809 & 2,5600 \\
\hline & 5 & 27,8153 & 26,7037 & 27,3067 & 27,3327 & 27,5667 & 0,2574 & $-0,1519$ & 1,9688 \\
\hline & 6 & 28,1608 & 26,5592 & 27,1407 & 27,0831 & 27,1663 & 0,3201 & 0,6853 & 2,9175 \\
\hline & 7 & 32,6311 & 30,9753 & 31,9235 & 31,9368 & 31,9803 & 0,3266 & $-0,5247$ & 2,8909 \\
\hline & 8 & 33,1992 & 31,8647 & 32,6568 & 32,7105 & 32,8257 & 0,3049 & $-0,8074$ & 3,1125 \\
\hline & 9 & 31,5378 & 30,0364 & 30,9003 & 30,8811 & 30,6760 & 0,3150 & $-0,0482$ & 2,4945 \\
\hline & 10 & 33,3934 & 31,8722 & 32,8480 & 32,9054 & 31,0276 & 0,2808 & $-0,5848$ & 2,8017 \\
\hline & 11 & 33,1563 & 32,1470 & 32,6834 & 32,6599 & 32,5806 & 0,2311 & $-0,0676$ & 2,2293 \\
\hline & 12 & 32,4913 & 30,2024 & 32,0495 & 32,0429 & 32,0647 & 0,2236 & $-2,6959$ & 20,6997 \\
\hline & 13 & 28,0269 & 26,7279 & 27,3980 & 27,3717 & 27,2962 & 0,2490 & 0,0520 & 2,4629 \\
\hline \multirow{13}{*}{$\frac{2}{2}$} & 1 & 34,2493 & 31,9731 & 32,7117 & 32,6095 & 32,5301 & 0,4387 & 1,1993 & 4,4869 \\
\hline & 2 & 33,3746 & 31,6606 & 32,6203 & 32,5533 & 32,5172 & 0,2811 & 0,1714 & 2,5976 \\
\hline & 3 & 33,7401 & 31,7480 & 32,9545 & 32,9503 & 32,9575 & 0,2852 & $-0,4021$ & 4,6211 \\
\hline & 4 & 29,4524 & 28,9548 & 29,2161 & 29,2150 & 29,2150 & 0,0827 & 0,2008 & 3,2241 \\
\hline & 5 & 26,9921 & 26,0176 & 26,5885 & 26,5973 & 26,4983 & 0,2146 & $-0,2251$ & 2,3509 \\
\hline & 6 & 25,9258 & 24,9185 & 25,3012 & 25,2655 & 25,1962 & 0,2177 & 0,5451 & 2,6524 \\
\hline & 7 & 31,6099 & 30,6163 & 31,2975 & 31,3332 & 31,3405 & 0,1795 & $-1,2540$ & 5,0888 \\
\hline & 8 & 33,2637 & 31,9953 & 32,7796 & 32,7897 & 32,8185 & 0,1860 & $-0,0597$ & 2,9159 \\
\hline & 9 & 31,8430 & 30,2647 & 31,2508 & 31,2391 & 31,1734 & 0,2582 & $-0,4214$ & 3,9464 \\
\hline & 10 & 33,9721 & 32,3719 & 33,4775 & 33,5294 & 33,8010 & 0,3385 & $-0,9874$ & 3,7468 \\
\hline & 11 & 33,3642 & 32,5012 & 32,9675 & 32,9731 & 33,1563 & 0,1944 & $-0,2671$ & 2,2227 \\
\hline & 12 & 33,0959 & 32,0140 & 32,5272 & 32,4480 & 32,2384 & 0,2856 & 0,1967 & 1,5220 \\
\hline & 13 & 28,6708 & 27,9593 & 28,3752 & 28,3942 & 28,3942 & 0,1193 & $-0,7521$ & 3,4630 \\
\hline \multicolumn{2}{|c|}{ Average } & 31,5822 & 30,2219 & 30,9749 & 30,9675 & 30,9000 & 0,2554 & $-0,2429$ & 3,7174 \\
\hline
\end{tabular}


$10.43^{\text {rd }}$ PIP Raw Data Measurements

Table 10-7: Raw Data Measurements Extracted from the Control Group's 3rd PIP Joint

\begin{tabular}{|c|c|c|c|c|c|c|c|c|c|}
\hline & & $\operatorname{Max}\left({ }^{\circ} \mathrm{C}\right)$ & $\operatorname{Min}\left({ }^{\circ} \mathrm{C}\right)$ & $\begin{array}{l}\text { Mean } \\
\left({ }^{\circ} \mathrm{C}\right)\end{array}$ & Median $\left({ }^{\circ} \mathrm{C}\right)$ & Mode $\left({ }^{\circ} \mathrm{C}\right)$ & STD & Skewness & Kurtosis \\
\hline \multirow{18}{*}{$\begin{array}{l}\text { 是 } \\
\text { 至 } \\
\text { 它 } \\
\frac{5}{2}\end{array}$} & 1 & 23,9560 & 22,8315 & 23,4545 & 23,4883 & 23,5784 & 0,2724 & $-0,2561$ & 2,0285 \\
\hline & 2 & 25,6722 & 23,4722 & 24,6071 & 24,7215 & 23,0000 & 0,5827 & $-0,2542$ & 1,9003 \\
\hline & 3 & 26,4327 & 25,7758 & 26,1384 & 26,1735 & 26,7914 & 0,1542 & $-0,4916$ & 2,2960 \\
\hline & 4 & 29,8958 & 29,1847 & 29,5182 & 29,4889 & 29,4074 & 0,1609 & 0,3444 & 2,0702 \\
\hline & 5 & 31,9230 & 31,0712 & 31,5652 & 31,5452 & 31,3412 & 0,1746 & $-0,0039$ & 2,3087 \\
\hline & 6 & 34,6756 & 33,5295 & 34,3252 & 34,3706 & 34,4629 & 0,2055 & $-0,8125$ & 3,2208 \\
\hline & 7 & 31,8943 & 30,6543 & 31,4381 & 31,5019 & 31,3781 & 0,2934 & $-0,7832$ & 2,8567 \\
\hline & 8 & 32,7824 & 31,2894 & 32,1107 & 32,1615 & 32,4074 & 0,3824 & $-0,3170$ & 1,9451 \\
\hline & 9 & $x$ & $x$ & $x$ & $\mathrm{x}$ & $x$ & $x$ & $x$ & $x$ \\
\hline & 10 & 32,9264 & 31,9948 & 32,6008 & 32,5842 & 32,5446 & 0,1580 & $-0,2833$ & 3,4714 \\
\hline & 11 & 27,4329 & 26,1121 & 26,6480 & 26,6150 & 26,3257 & 0,2813 & 0,5239 & 2,5163 \\
\hline & 12 & 26,7825 & 24,8989 & 25,6722 & 25,5841 & 25,4152 & 0,4094 & 0,8409 & 3,0632 \\
\hline & 13 & 20,8057 & 19,9920 & 20,3610 & 20,3392 & 20,5808 & 0,2136 & 0,2387 & 1,8638 \\
\hline & 14 & 25,0377 & 22,5075 & 24,4511 & 24,4955 & 24,6901 & 0,3179 & $-0,5689$ & 2,6252 \\
\hline & 15 & 33,5939 & 32,6392 & 33,2103 & 33,2574 & 33,3148 & 0,2038 & $-0,4816$ & 2,4218 \\
\hline & 16 & 29,8668 & 29,2224 & 29,5288 & 29,5266 & 29,4376 & 0,1231 & 0,0798 & 2,2274 \\
\hline & 17 & 32,2917 & 30,8289 & 31,7511 & 31,7590 & 31,5153 & 0,2921 & $-0,3078$ & 2,4830 \\
\hline & 18 & 33,0563 & 32,5820 & 32,4068 & 32,4152 & 32,5380 & 0,3051 & 0,0117 & 2,0030 \\
\hline \multirow{18}{*}{ 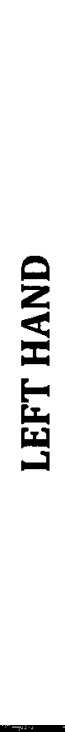 } & 1 & 25,0723 & 24,1567 & 24,7379 & 24,7783 & 24,7860 & 0,1483 & $-1,2740$ & 4,8236 \\
\hline & 2 & 25,7799 & 23,7154 & 24,8530 & 24,8526 & 24,0830 & 0,0890 & 0,0883 & 1,9048 \\
\hline & 3 & 25,1381 & 24,4423 & 24,8512 & 24,8526 & 24,8526 & 0,0928 & $-0,5075$ & 4,8054 \\
\hline & 4 & 27,9743 & 26,8418 & 27,3029 & 27,2659 & 27,2130 & 0,1943 & 1,1226 & 4,5468 \\
\hline & 5 & 33,0563 & 31,8432 & 32,6148 & 32,6317 & 32,8190 & 0,2018 & $-0,5266$ & 3,1801 \\
\hline & 6 & 34,7534 & 32,3938 & 34,2317 & 34,3777 & 34,5267 & 0,4509 & $-2,0826$ & 7,2341 \\
\hline & 7 & 32,2131 & 31,1665 & 31,8101 & 31,8145 & 31,7709 & 0,2198 & $-0,3384$ & 2,3954 \\
\hline & 8 & 32,5518 & 31,3988 & 32,0127 & 32,0311 & 32,1035 & 0,2789 & $-0,2012$ & 2,1068 \\
\hline & 9 & $x$ & $x$ & $x$ & $x$ & $\mathrm{x}$ & $x$ & $x$ & $\mathrm{x}$ \\
\hline & 10 & 33,0916 & 32,4579 & 32,7568 & 32,7680 & 32,7608 & 0,1255 & $-0,1983$ & 2,4668 \\
\hline & 11 & 27,0020 & 26,0052 & 26,5575 & 26,5542 & 26,5846 & 0,1520 & $-0,0588$ & 3,8001 \\
\hline & 12 & 27,5088 & 25,5534 & 26,5039 & 26,5241 & 26,7445 & 0,4317 & 0,1170 & 2,1884 \\
\hline & 13 & 21,1503 & 20,4278 & 20,7717 & 20,7656 & 20,6731 & 0,1521 & 0,1211 & 2,1674 \\
\hline & 14 & 24,6669 & 23,0842 & 24,1386 & 24,2248 & 24,2870 & 0,3350 & $-0,5925$ & 2,5137 \\
\hline & 15 & 33,1929 & 31,9596 & 32,8297 & 32,8408 & 32,9900 & 0,2003 & $-0,6432$ & 3,5083 \\
\hline & 16 & 30,6763 & 27,9901 & 30,0173 & 30,0439 & 30,0734 & 0,3815 & $-1,1189$ & 8,8599 \\
\hline & 17 & 32,2194 & 30,6750 & 31,5092 & 31,5736 & 31,6099 & 0,3830 & $-0,2397$ & 2,1127 \\
\hline & 18 & 32,4586 & 29,8515 & 31,9046 & 31,9883 & 32,1260 & 0,3742 & $-1,6559$ & 6,5996 \\
\hline \multicolumn{2}{|c|}{ Average } & 29,3392 & 28,0162 & 28,7997 & 28,8210 & 28,7863 & 0,2571 & $-0,3091$ & 3,1328 \\
\hline
\end{tabular}


Table 10-8: Raw Data Measurements Extracted from the Patient Group's 3rd PIP Joint

\begin{tabular}{|c|c|c|c|c|c|c|c|c|c|}
\hline & & $\operatorname{Max}\left({ }^{\circ} \mathrm{C}\right)$ & $\operatorname{Min}\left({ }^{\circ} \mathrm{C}\right)$ & $\begin{array}{c}\text { Mean } \\
\left({ }^{\circ} \mathrm{C}\right)\end{array}$ & Median ( ${ }^{\circ} \mathrm{C}$ ) & Mode $\left({ }^{\circ} \mathrm{C}\right)$ & STD & Skewness & Kurtosis \\
\hline \multirow{13}{*}{ 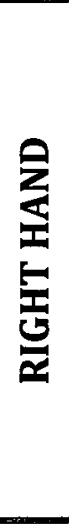 } & 1 & 33,5289 & 32,8184 & 33,0773 & 33,0773 & 33,0342 & 0,1357 & 0,6941 & 3,4272 \\
\hline & 2 & 34,2328 & 33,4104 & 33,7615 & 33,7615 & 33,6685 & 0,1808 & 0,3197 & 2,4467 \\
\hline & 3 & 33,4104 & 32,0824 & 32,5392 & 32,5027 & 32,4304 & 0,2007 & 0,9315 & 4,0332 \\
\hline & 4 & 30,0953 & 29,5561 & 29,5667 & 29,5635 & 29,5561 & 0,1535 & 0,1560 & 3,3237 \\
\hline & 5 & 28,2658 & 26,7645 & 27,6347 & 27,6534 & 27,7023 & 0,3175 & $-0,2577$ & 2,6487 \\
\hline & 6 & 28,5202 & 27,5365 & 27,8068 & 27,7475 & 27,7174 & 0,1799 & 1,1686 & 4,1105 \\
\hline & 7 & 32,6960 & 30,3792 & 31,6920 & 31,7335 & 32,1470 & 0,4482 & $-0,6188$ & 3,2032 \\
\hline & 8 & 33,3712 & 32,0894 & 32,8327 & 32,9551 & 33,0557 & 0,3367 & $-0,5929$ & 1,9303 \\
\hline & 9 & 32,0605 & 30,9615 & 31,6659 & 31,7123 & 31,7922 & 0,2169 & $-0,6887$ & 2,8885 \\
\hline & 10 & 33,3791 & 31,7924 & 32,8316 & 32,8838 & 32,9054 & 0,3019 & $-0,9411$ & 3,6980 \\
\hline & 11 & 33,1921 & 31,9803 & 32,8174 & 32,8328 & 33,0342 & 0,2254 & $-0,5874$ & 2,8505 \\
\hline & 12 & 32,4263 & 32,5993 & 31,9120 & 31,9415 & 31,9850 & 0,2403 & $-0,7377$ & 5,1512 \\
\hline & 13 & 28,3343 & 27,0086 & 27,9117 & 28,0194 & 28,1245 & 0,2729 & $-1,3696$ & 4,1838 \\
\hline \multirow{13}{*}{$\frac{2}{\mathbf{Z}}$} & 1 & 33,3141 & 32,2339 & 32,6633 & 32,6383 & 32,6599 & 0,1754 & 0,7078 & 3,7320 \\
\hline & 2 & 33,7258 & 32,1405 & 33,0166 & 33,0295 & 33,0655 & 0,2652 & $-0,0546$ & 3,7256 \\
\hline & 3 & 33,1446 & 27,3540 & 32,3982 & 32,4882 & 32,5389 & 0,5399 & $-3,5814$ & 24,3273 \\
\hline & 4 & 30,0585 & 28,6566 & 29,7749 & 29,8076 & 29,8224 & 0,1941 & $-3,2520$ & 16,9999 \\
\hline & 5 & 27,7701 & 26,5364 & 27,3070 & 27,3176 & 27,1739 & 0,2133 & $-0,5223$ & 3,4431 \\
\hline & 6 & 26,6505 & 24,9803 & 25,6087 & 25,5770 & 25,4886 & 0,3359 & 0,4455 & 2,6146 \\
\hline & 7 & 31,6318 & 30,4547 & 31,0550 & 31,0850 & 31,1872 & 0,2206 & $-0,2871$ & 2,8017 \\
\hline & 8 & 33,1275 & 31,9300 & 32,7294 & 32,7897 & 32,7969 & 0,2414 & $-1,4349$ & 4,6900 \\
\hline & 9 & 32,4293 & 30,9908 & 31,6931 & 31,7195 & 31,7413 & 0,2545 & $-0,3136$ & 2,9421 \\
\hline & 10 & 34,2071 & 32,0028 & 33,7279 & 33,7795 & 33,8723 & 0,3043 & $-1,2187$ & 6,0979 \\
\hline & 11 & 33,5861 & 31,7190 & 33,0883 & 33,0916 & 32,9623 & 0,2846 & $-1,4494$ & 7,2922 \\
\hline & 12 & 33,1892 & 30,7606 & 32,3940 & 32,3541 & 32,3035 & 0,3465 & 0,0880 & 4,4325 \\
\hline & 13 & 28,8498 & 28,1170 & 28,5922 & 28,6110 & 28,6708 & 0,1167 & $-1,5937$ & 6,6777 \\
\hline \multicolumn{2}{|c|}{ Average } & 31,7384 & 30,2637 & 31,1576 & 31,1797 & 31,2091 & 0,2578 & $-0,5766$ & 5,1412 \\
\hline
\end{tabular}




\subsection{Wrist Raw Data Measurements}

Table 10-9: Raw Data Measurements Extracted from the Control Group's Wrist Joint

\begin{tabular}{|c|c|c|c|c|c|c|c|c|c|}
\hline & & $\operatorname{Max}\left({ }^{\circ} \mathrm{C}\right)$ & $\operatorname{Min}\left({ }^{\circ} \mathrm{C}\right)$ & $\begin{array}{c}\text { Mean } \\
\text { ('C) }\end{array}$ & Median $\left({ }^{\circ} \mathrm{C}\right)$ & Mode $\left({ }^{\circ} \mathrm{C}\right)$ & STD & Skewness & Kurtosis \\
\hline \multirow{18}{*}{$\underset{⿱ 乛}{\mathbf{E}}$} & 1 & 30,2050 & 27,7589 & 28,9412 & 28,9812 & 29,1451 & 0,4239 & 0,0795 & 3,1567 \\
\hline & 2 & 30,6159 & 29,5064 & 30,2165 & 30,2694 & 30,4170 & 0,2143 & $-0,6369$ & 2,5121 \\
\hline & 3 & 30,0806 & 28,8356 & 29,5489 & 29,5709 & 29,5857 & 0,2497 & $-0,2504$ & 2,4466 \\
\hline & 4 & 31,2099 & 29,5630 & 30,4687 & 30,5511 & 30,6905 & 0,3239 & $-0,6040$ & 2,7330 \\
\hline & 5 & 32,5668 & 30,9322 & 31,7652 & 31,7851 & 31,8505 & 0,2472 & $-0,3625$ & 4,4286 \\
\hline & 6 & 34,2000 & 32,5671 & 33,5556 & 33,5653 & 33,4365 & 0,2638 & $-0,4692$ & 3,4082 \\
\hline & 7 & 32,0828 & 30,3458 & 31,4071 & 31,4946 & 31,5237 & 0,3505 & $-1,0118$ & 3,5709 \\
\hline & 8 & 31,8425 & 30,1236 & 31,1865 & 31,2164 & 31,2967 & 0,3055 & 0,3055 & 3,1960 \\
\hline & 9 & $x$ & $x$ & $x$ & $x$ & $\mathrm{x}$ & $x$ & $x$ & $\mathbf{x}$ \\
\hline & 10 & 32,2845 & 31,0046 & 31,6791 & 31,6972 & 31,9658 & 0,2580 & $-0,2979$ & 2,2263 \\
\hline & 11 & 29,5999 & 27,2970 & 28,5659 & 28,5740 & 27,9674 & 0,5275 & $-0,0042$ & 1,7807 \\
\hline & 12 & 30,2205 & 27,4635 & 28,9351 & 29,0887 & 29,3486 & 0,5902 & $-0,2877$ & 1,9391 \\
\hline & 13 & 27,7945 & 26,2342 & 27,1734 & 27,2593 & 27,2366 & 0,3430 & $-0,5907$ & 2,2589 \\
\hline & 14 & 29,2812 & 27,3197 & 28,6492 & 28,7531 & 29,0361 & 0,3945 & $-1,0059$ & 3,3200 \\
\hline & 15 & 33,2933 & 31,3489 & 32,4388 & 32,4588 & 32,2637 & 0,4179 & $-0,2118$ & 2,2084 \\
\hline & 16 & 29,6524 & 27,6592 & 28,8663 & 28,8951 & 28,8355 & 0,3558 & $-0,9410$ & 4,2062 \\
\hline & 17 & 32,1687 & 30,2561 & 31,4165 & 31,4206 & 31,3769 & 0,3449 & $-0,6344$ & 3,7072 \\
\hline & 18 & 33,0635 & 31,3777 & 32,0878 & 32,0137 & 31,7343 & 0,3965 & 0,3513 & 1,9741 \\
\hline \multirow{18}{*}{$\sum_{\substack{1 \\
y}}^{2}$} & 1 & 30,2935 & 28,7572 & 29,1285 & 29,1004 & 29,1153 & 0,1931 & 2,9045 & 15,2369 \\
\hline & 2 & 31,1520 & 29,3650 & 30,2741 & 30,2768 & 30,2916 & 0,4312 & 0,0313 & 1,9514 \\
\hline & 3 & 29,2670 & 28,3577 & 28,7009 & 28,7014 & 28,7089 & 0,1144 & 0,2780 & 4,0285 \\
\hline & 4 & 30,5511 & 29,3184 & 29,9968 & 30,0655 & 30,3233 & 0,2947 & $-0,3467$ & 1,7962 \\
\hline & 5 & 32,8622 & 30,7418 & 32,1231 & 32,2273 & 32,4586 & 0,4351 & $-1,0711$ & 3,4849 \\
\hline & 6 & 34,6401 & 32,6319 & 33,6439 & 33,6332 & 33,4580 & 0,3945 & $-0,0707$ & 2,3315 \\
\hline & 7 & 32,9990 & 30,9473 & 32,1186 & 32,2565 & 32,3432 & 0,4612 & $-0,5305$ & 2,4064 \\
\hline & 8 & 32,6095 & 29,7544 & 30,4107 & 30,3739 & 30,4621 & 0,4568 & 1,5358 & 6,3220 \\
\hline & 9 & $\mathrm{x}$ & $x$ & $x$ & $\mathrm{x}$ & $x$ & $x$ & $x$ & $x$ \\
\hline & 10 & 32,6744 & 30,7336 & 31,7385 & 31,7481 & 31,7626 & 0,4049 & $-0,0603$ & 2,2975 \\
\hline & 11 & 29,2293 & 26,9565 & 28,1030 & 28,0875 & 27,7042 & 0,4584 & 0,1314 & 1,9942 \\
\hline & 12 & 29,9110 & 27,9303 & 28,8386 & 28,6939 & 28,5894 & 0,4914 & 0,2377 & 1,7607 \\
\hline & 13 & 27,7795 & 26,3791 & 27,1845 & 27,2593 & 27,2819 & 0,2472 & $-0,8275$ & 3,2881 \\
\hline & 14 & 29,4296 & 27,3499 & 28,6857 & 28,8500 & 29,0807 & 0,4406 & $-0,7102$ & 2,3449 \\
\hline & 15 & 33,4437 & 31,3781 & 32,5612 & 32,6103 & 32,5815 & 0,4291 & $-0,4379$ & 2,4750 \\
\hline & 16 & 29,7781 & 27,9225 & 29,1167 & 29,1110 & 29,0292 & 0,3353 & $-0,9689$ & 4,4678 \\
\hline & 17 & 32,2121 & 30,8948 & 31,4655 & 31,3842 & 31,3186 & 0,2606 & 0,7813 & 2,8732 \\
\hline & 18 & 33,3648 & 31,9158 & 32,7281 & 32,7686 & 32,7686 & 0,2658 & $-0,4532$ & 2,6065 \\
\hline \multicolumn{2}{|c|}{ Average } & 31,2459 & 29,4391 & 30,4035 & 30,4336 & 30,4408 & 0,3565 & $-0,1809$ & 3,2570 \\
\hline
\end{tabular}


Table 10-10: Raw Data Measurements Extracted from the Patient Group's Wrist Joint

\begin{tabular}{|c|c|c|c|c|c|c|c|c|c|}
\hline & & $\operatorname{Max}\left({ }^{\circ} \mathrm{C}\right)$ & $\operatorname{Min}\left({ }^{\circ} \mathrm{C}\right)$ & $\begin{array}{l}\text { Mean } \\
\left({ }^{\circ} \mathrm{C}\right)\end{array}$ & Median ( ${ }^{\circ} \mathrm{C}$ ) & Mode ( $\left.{ }^{\circ} \mathrm{C}\right)$ & STD & Skewness & Kurtosis \\
\hline \multirow{13}{*}{$\frac{Q}{2}$} & 1 & 34,2066 & 32,1977 & 33,1474 & 33,1634 & 33,2137 & 0,3134 & 0,0215 & 2,7879 \\
\hline & 2 & 34,2684 & 32,3145 & 33,5902 & 33,6757 & 33,7472 & 0,3773 & $-0,7149$ & 2,7312 \\
\hline & 3 & 33,2884 & 31,1056 & 32,3249 & 32,3797 & 32,4665 & 0,4096 & $-0,4979$ & 2,7675 \\
\hline & 4 & 29,9847 & 28,7685 & 29,3541 & 29,3338 & 29,3189 & 0,2148 & 0,3113 & 3,1018 \\
\hline & 5 & 31,7263 & 30,1088 & 31,1276 & 31,2383 & 31,2967 & 0,3623 & $-0,6427$ & 2,2889 \\
\hline & 6 & 33,9503 & 32,1398 & 33,1905 & 33,1921 & 33,1706 & 0,3524 & $-0,1293$ & 2,5688 \\
\hline & 7 & 34,7956 & 32,1615 & 33,6762 & 33,7576 & 33,8647 & 0,4042 & $-0,5392$ & 3,2532 \\
\hline & 8 & 32,6456 & 30,8152 & 31,4377 & 31,4067 & 31,5014 & 0,3320 & 0,5215 & 2,7766 \\
\hline & 9 & 31,2318 & 29,0143 & 29,9105 & 29,7854 & 29,5117 & 0,4732 & 0,8039 & 2,7549 \\
\hline & 10 & 33,8366 & 32,1549 & 33,0564 & 33,0635 & 32,9413 & 0,3304 & $-0,2092$ & 2,4490 \\
\hline & 11 & 33,1347 & 31,4425 & 32,3925 & 32,2990 & 32,1904 & 0,3104 & 0,2612 & 2,2979 \\
\hline & 12 & 32,5562 & 30,7533 & 31,8807 & 31,9125 & 31,9632 & 0,3151 & $-0,6963$ & 3,3497 \\
\hline & 13 & 32,7470 & 29,4074 & 30,8240 & 30,6978 & 30,5951 & 0,6755 & 0,5330 & 2,9259 \\
\hline \multirow{13}{*}{$\underset{\square}{E}$} & 1 & 33,7648 & 31,9296 & 32,9132 & 32,9479 & 33,2424 & 0,4176 & $-0,1749$ & 1,9664 \\
\hline & 2 & 33,3746 & 31,8063 & 32,6345 & 32,6617 & 32,7267 & 0,2394 & $-0,3333$ & 3,3226 \\
\hline & 3 & 33,9831 & 31,4054 & 33,2195 & 33,3530 & 33,3961 & 0,4666 & $-1,5500$ & 4,7620 \\
\hline & 4 & 29,9995 & 28,3801 & 29,0712 & 29,0441 & 28,9027 & 0,2831 & 0,2483 & 2,8239 \\
\hline & 5 & 30,6310 & 29,2728 & 30,0485 & 30,0720 & 30,1088 & 0,1830 & $-0,3235$ & 3,3593 \\
\hline & 6 & 31,0484 & 28,6697 & 29,6175 & 29,5546 & 29,4879 & 0,3722 & 0,5551 & 3,0703 \\
\hline & 7 & 34,5901 & 32,7464 & 33,6430 & 33,6219 & 33,7005 & 0,4419 & 0,0778 & 2,0832 \\
\hline & 8 & 30,3382 & 28,2529 & 29,3050 & 29,3115 & 29,3115 & 0,4425 & 0,0030 & 2,3523 \\
\hline & 9 & 31,2391 & 28,8580 & 30,1481 & 30,1764 & 30,5219 & 0,5025 & $-0,3939$ & 2,5175 \\
\hline & 10 & 34,2711 & 32,1042 & 33,1426 & 33,1066 & 33,7153 & 0,5357 & 0,0518 & 1,7211 \\
\hline & 11 & 33,7434 & 31,9948 & 33,0708 & 33,1060 & 33,1634 & 0,2512 & $-0,5258$ & 3,4519 \\
\hline & 12 & 33,5473 & 30,4451 & 32,2599 & 32,3541 & 32,6643 & 0,5759 & $-0,8582$ & 3,6166 \\
\hline & 13 & 32,3936 & 30,2938 & 31,4909 & 31,5670 & 31,7851 & 0,4633 & $-0,4238$ & 2,2843 \\
\hline \multicolumn{2}{|c|}{ Average } & 32,7422 & 30,7132 & 31,7876 & 31,7993 & 31,8657 & 0,3864 & $-0,1779$ & 2,8225 \\
\hline
\end{tabular}


10.6 Knee Raw Data Measurements

Table 10-11: Raw Data Measurements Extracted from the Control Group's Knee Joint

\begin{tabular}{|c|c|c|c|c|c|c|c|c|c|}
\hline & & $\operatorname{Max}\left({ }^{\circ} \mathrm{C}\right)$ & $\operatorname{Min}\left({ }^{\circ} \mathrm{C}\right)$ & $\begin{array}{c}\text { Mean } \\
\left({ }^{\circ} \mathrm{C}\right)\end{array}$ & Median ( $\left.{ }^{\circ} \mathrm{C}\right)$ & Mode $\left({ }^{\circ} \mathrm{C}\right)$ & STD & Skewness & Kurtosis \\
\hline \multirow{18}{*}{ 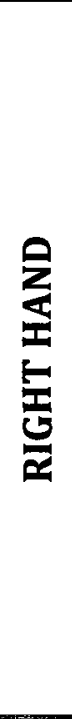 } & 1 & 30,2493 & 27,0934 & 28,7182 & 28,8543 & 29,0781 & 0,5696 & $-0,3387$ & 2,7822 \\
\hline & 2 & 29,8330 & 27,8514 & 29,1100 & 29,1639 & 29,3204 & 0,3243 & $-1,0887$ & 4,4812 \\
\hline & 3 & 30,3823 & 27,6369 & 29,6064 & 29,6745 & 29,7189 & 0,4351 & $-1,5978$ & 6,4680 \\
\hline & 4 & 33,0060 & 28,4765 & 31,0202 & 31,1515 & 31,4141 & 0,8399 & $-0,4804$ & 2,9319 \\
\hline & 5 & 31,3194 & 28,8498 & 30,3849 & 30,4923 & 30,6025 & 0,4187 & $-0,9371$ & 3,9023 \\
\hline & 6 & 31,9233 & 28,7535 & 30,9183 & 31,0716 & 31,3124 & 0,5506 & $-1,0887$ & 3,7233 \\
\hline & 7 & 31,1884 & 28,4174 & 29,9771 & 29,9628 & 29,9037 & 0,5265 & $-0,3303$ & 2,8037 \\
\hline & 8 & 32,3423 & 30,2193 & 31,3156 & 31,3332 & 31,3769 & 0,3390 & $-0,2411$ & 2,8205 \\
\hline & 9 & 33,0342 & 30,2267 & 31,8757 & 31,8715 & 31,7771 & 0,4745 & $-0,1384$ & 2,7094 \\
\hline & 10 & 30,9168 & 28,7518 & 29,5376 & 29,4879 & 29,4435 & 0,2537 & 1,1037 & 5,7005 \\
\hline & 11 & 30,6826 & 26,7517 & 29,4577 & 29,6591 & 29,7035 & 0,7381 & $-1,1407$ & 3,7774 \\
\hline & 12 & 30,9250 & 26,6305 & 29,0497 & 29,1184 & 28,7909 & 0,9487 & $-0,2888$ & 2,1461 \\
\hline & 13 & 28,7083 & 25,6913 & 27,7761 & 27,9298 & 28,2299 & 0,6067 & $-1,0905$ & 3,6389 \\
\hline & 14 & 30,6606 & 26,1121 & 29,2306 & 29,3109 & 29,4147 & 0,5991 & $-0,7396$ & 3,6096 \\
\hline & 15 & 29,6450 & 28,4024 & 29,1597 & 29,1556 & 29,1110 & 0,1573 & $-0,2628$ & 3,6784 \\
\hline & 16 & 32,1769 & 28,3350 & 30,6243 & 30,7056 & 31,4509 & 0,9255 & $-0,3442$ & 2,1790 \\
\hline & 17 & 30,9753 & 28,2957 & 29,7534 & 29,8431 & 30,0277 & 0,5805 & $-0,2536$ & 2,1453 \\
\hline & 18 & 31,7626 & 28,6323 & 29,9560 & 30,0130 & 30,0425 & 0,5676 & $-0,0333$ & 2,5365 \\
\hline \multirow{18}{*}{$\frac{2}{2}$} & 1 & 31,9546 & 28,1278 & 30,1671 & 30,0943 & 29,8948 & 0,5649 & 0,252 & 2,9179 \\
\hline & 2 & 31,2692 & 28,4383 & 29,6503 & 29,6549 & 29,6921 & 0,3858 & $-0,0075$ & 3,5097 \\
\hline & 3 & 30,5880 & 27,3428 & 29,5864 & 29,6375 & 29,6523 & 0,435 & $-1,4072$ & 6,3791 \\
\hline & 4 & 32,6894 & 29,0360 & 31,0510 & 31,2172 & 31,5961 & 0,7624 & $-0,4195$ & 2,4765 \\
\hline & 5 & 31,2099 & 27,9969 & 29,7904 & 29,8810 & 30,3086 & 0,6447 & $-0,3787$ & 2,5655 \\
\hline & 6 & 32,3288 & 29,2670 & 31,0810 & 31,1884 & 31,3562 & 0,5707 & $-0,5929$ & 2,7631 \\
\hline & 7 & 31,9523 & 28,4248 & 30,0810 & 30,0955 & 30,6396 & 0,6653 & $-0,031$ & 2,3417 \\
\hline & 8 & 32,5085 & 29,9613 & 31,1837 & 31,2383 & 31,3186 & 0,4801 & $-0,1617$ & 2,4846 \\
\hline & 9 & 32,7608 & 29,8136 & 31,6116 & 31,6390 & 31,6536 & 0,4803 & $-0,6058$ & 3,5678 \\
\hline & 10 & 30,5429 & 28,5427 & 29,4554 & 29,3693 & 29,3619 & 0,3285 & 0,8274 & 3,2571 \\
\hline & 11 & 30,1904 & 26,1274 & 28,6153 & 28,8127 & 29,4147 & 0,8386 & $-0,7738$ & 2,8185 \\
\hline & 12 & 31,3339 & 25,9748 & 28,9712 & 29,0738 & 29,8297 & 1,0518 & $-0,3539$ & 2,2779 \\
\hline & 13 & 29,8069 & 26,0968 & 28,4322 & 28,5889 & 28,8574 & 0,6943 & $-0,8351$ & 3,2502 \\
\hline & 14 & 30,6679 & 26,5846 & 29,1372 & 29,2738 & 29,5185 & 0,7781 & $-0,6062$ & 2,7147 \\
\hline & 15 & 31,3489 & 27,9526 & 29,0225 & 28,9696 & 28,8430 & 0,3935 & 0,7195 & 4,7714 \\
\hline & 16 & 31,7637 & 28,8057 & 30,5102 & 30,5222 & 30,3752 & 0,6596 & $-0,2578$ & 2,26 \\
\hline & 17 & 31,4511 & 29,1911 & 30,4602 & 30,4841 & 30,7556 & 0,4091 & $-0,3273$ & 2,7163 \\
\hline & 18 & 32,5668 & 30,1332 & 31,8815 & 31,9448 & 32,1332 & 0,307 & $-0,5449$ & 2,6813 \\
\hline \multicolumn{2}{|c|}{ Average } & 31,2963 & 28,1374 & 29,9489 & 30,0135 & 30,1644 & 0,5640 & $-0,4110$ & 3,2719 \\
\hline
\end{tabular}


Table 10-12: Raw Data Measurements Extracted from the Patient Group's Knee Joint

\begin{tabular}{|c|c|c|c|c|c|c|c|c|c|}
\hline & & $\operatorname{Max}\left({ }^{\circ} \mathrm{C}\right)$ & $\operatorname{Min}\left({ }^{\circ} \mathrm{C}\right)$ & $\begin{array}{c}\text { Mean } \\
\text { ('C) }\end{array}$ & Median ( ${ }^{\circ} \mathrm{C}$ ) & Mode $\left({ }^{\circ} \mathrm{C}\right)$ & STD & Skewness & Kurtosis \\
\hline \multirow{13}{*}{ 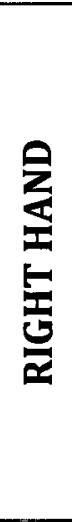 } & 1 & 31,8352 & 28,9828 & 30,6760 & 30,7776 & 31,0996 & 0,5633 & $-0,6414$ & 2,8053 \\
\hline & 2 & 31,6898 & 29,8763 & 30,7146 & 30,7316 & 30,8564 & 0,3305 & $-0,1307$ & 2,4253 \\
\hline & 3 & 31,8352 & 28,9828 & 30,6760 & 30,7776 & 31,0996 & 0,5633 & $-0,6414$ & 2,8053 \\
\hline & 4 & 29,0738 & 25,9060 & 28,0741 & 28,1629 & 28,2454 & 0,5395 & $-1,1527$ & 4,6386 \\
\hline & 5 & 30,7923 & 28,6024 & 30,0984 & 30,2120 & 30,5576 & 0,4687 & $-0,7398$ & 2,6766 \\
\hline & 6 & 31,2748 & 28,6398 & 30,1301 & 30,1751 & 30,5796 & 0,4721 & $-0,4066$ & 2,3680 \\
\hline & 7 & 32,0746 & 30,6530 & 31,3990 & 31,4206 & 31,5081 & 0,2433 & $-0,1090$ & 2,6753 \\
\hline & 8 & 29,8076 & 27,6595 & 28,7702 & 28,7760 & 28,8878 & 0,3868 & 0,1107 & 2,5216 \\
\hline & 9 & 29,0441 & 26,7597 & 27,9101 & 27,8551 & 27,7574 & 0,4155 & 0,4489 & 2,7057 \\
\hline & 10 & 31,9810 & 29,9032 & 31,1506 & 31,1880 & 31,4214 & 0,3226 & $-0,5389$ & 3,0899 \\
\hline & 11 & 30,4033 & 28,9828 & 29,9602 & 30,0130 & 30,0720 & 0,2136 & $-1,2839$ & 4,6348 \\
\hline & 12 & 31,2724 & 28,9586 & 30,2112 & 30,2908 & 30,4745 & 0,3652 & $-0,2989$ & 2,3884 \\
\hline & 13 & 30,5438 & 28,1095 & 29,3482 & 29,3480 & 29,3184 & 0,3617 & $-0,1871$ & 3,5468 \\
\hline \multirow{13}{*}{$\frac{9}{\mathbb{Z}}$} & 1 & 32,1325 & 29,2579 & 31,0218 & 31,1872 & 31,3988 & 0,6037 & $-0,7395$ & 2,71 \\
\hline & 2 & 31,4200 & 29,5205 & 30,5332 & 30,5110 & 30,4669 & 0,3355 & $-0,0295$ & 2,64 \\
\hline & 3 & 31,8645 & 29,4685 & 30,5154 & 30,4742 & 29,8097 & 0,5694 & 0,2446 & 1,9134 \\
\hline & 4 & 28,1704 & 25,5611 & 27,3531 & 27,4409 & 27,6821 & 0,4235 & $-1,1194$ & 4,3429 \\
\hline & 5 & 31,0850 & 28,7592 & 29,8999 & 29,9170 & 29,8801 & 0,4175 & $-0,1652$ & 2,5133 \\
\hline & 6 & 31,0484 & 28,4754 & 30,0377 & 30,1751 & 30,3297 & 0,5569 & $-0,5574$ & 2,4235 \\
\hline & 7 & 31,1434 & 29,3693 & 30,4272 & 30,4474 & 30,4400 & 0,2915 & $-0,5222$ & 3,2053 \\
\hline & 8 & 31,6323 & 27,1840 & 29,2335 & 29,1704 & 27,1840 & 0,9486 & 0,073 & 2,0262 \\
\hline & 9 & 28,8878 & 26,9493 & 28,0124 & 28,0279 & 27,9979 & 0,3666 & $-0,2073$ & 2,7337 \\
\hline & 10 & 31,7561 & 22,8927 & 29,5297 & 30,5878 & 30,4703 & 2,5207 & $-1,5207$ & 4,4411 \\
\hline & 11 & 31,5372 & 29,4583 & 30,2717 & 30,2414 & 30,1457 & 0,3803 & 0,3999 & 2,7651 \\
\hline & 12 & 32,7652 & 29,1966 & 30,9183 & 30,9437 & 31,0972 & 0,7165 & $-0,0808$ & 2,4402 \\
\hline & 13 & 31,3485 & 28,1470 & 29,4361 & 29,4000 & 29,3555 & 0,4705 & 0,8634 & 4,9584 \\
\hline \multicolumn{2}{|c|}{ Average } & 31,0161 & 28,3175 & 29,8580 & 29,9328 & 29,9283 & 0,5326 & $-0,3435$ & 3,0152 \\
\hline
\end{tabular}




\section{APPENDIX E}

11.1 Histogram of $2^{\text {nd }}$ MCP's Most Statistically Significant Matrices

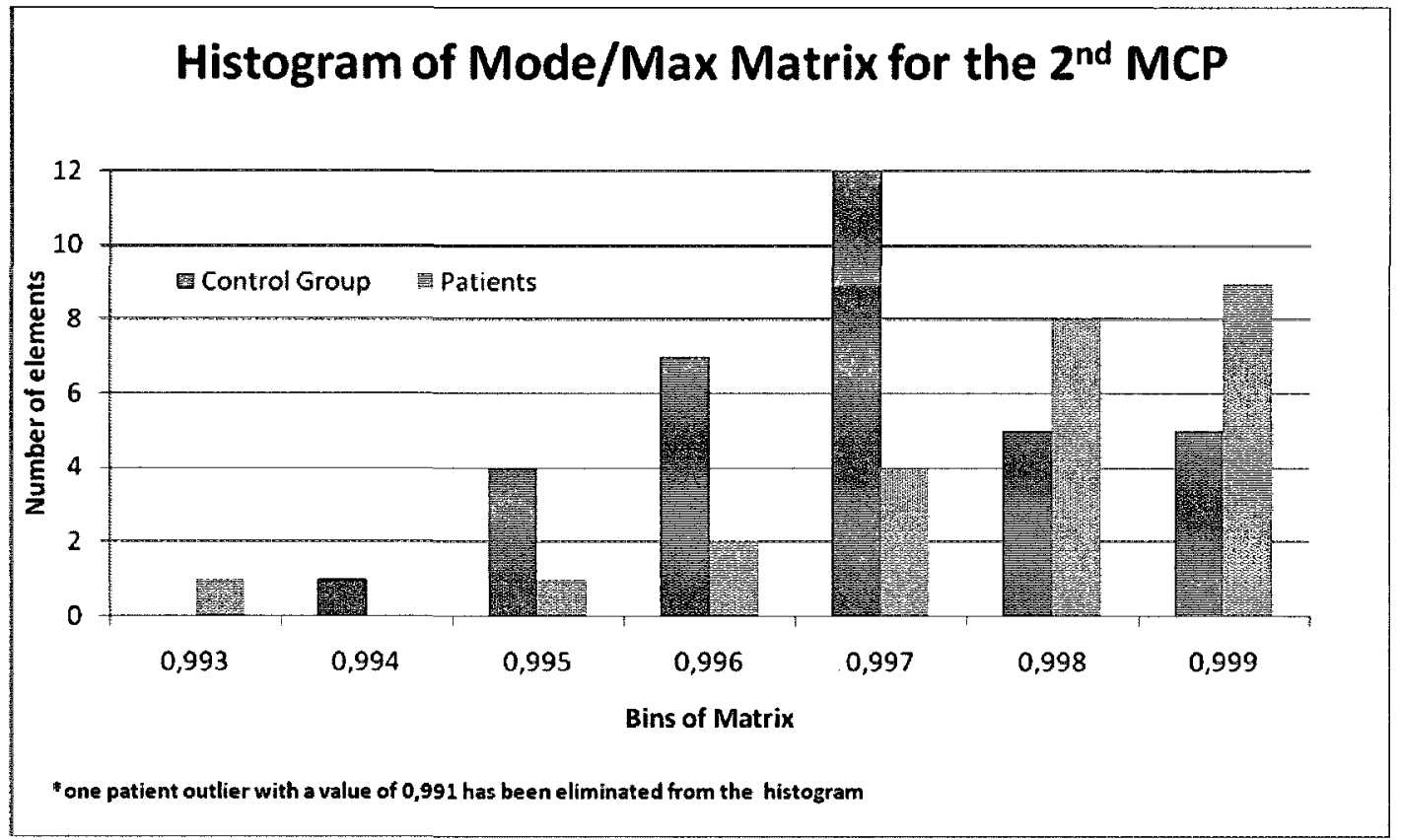

Figure 11-1: Histogram of the Mode/Max Matrix for the 2nd MCP 


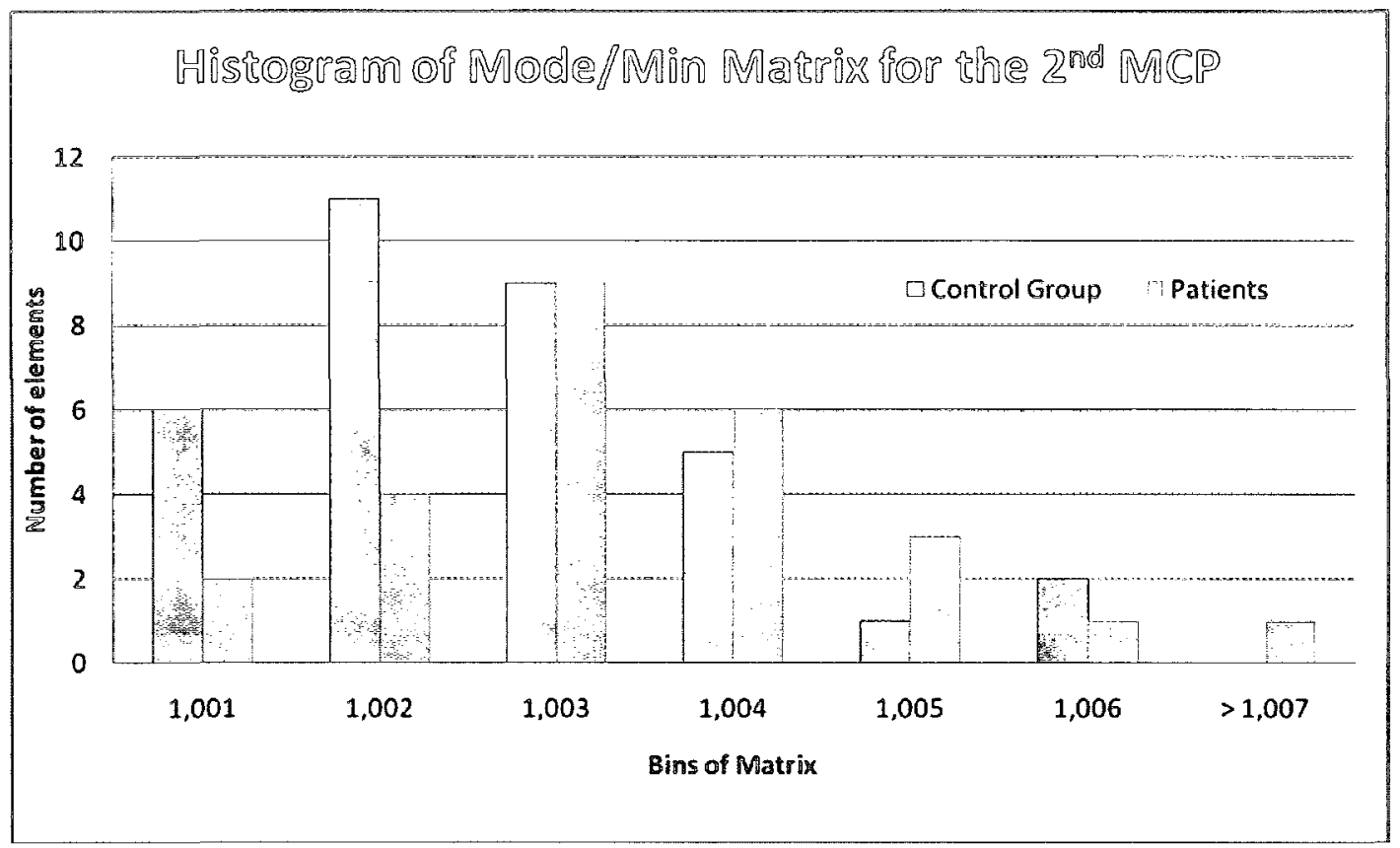

Figure 11-2: Histogram of the Mode/Min Matrix for the 2nd MCP

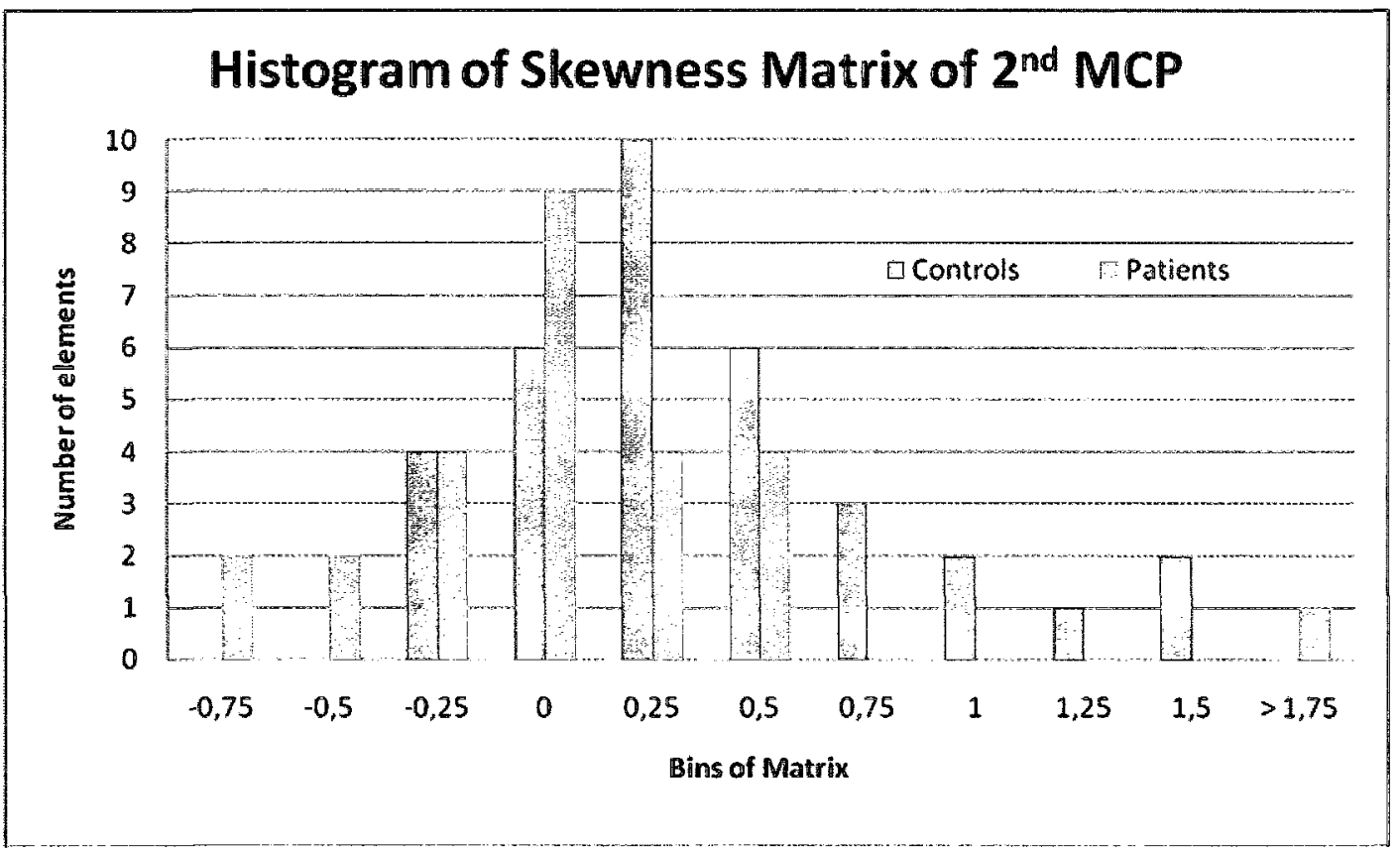

Figure 11-3: Histogram of the Skewness Matrix for the 2nd MCP 
11.2 Histogram of $3^{r d}$ MCP's Most Statistically Significant Matrices

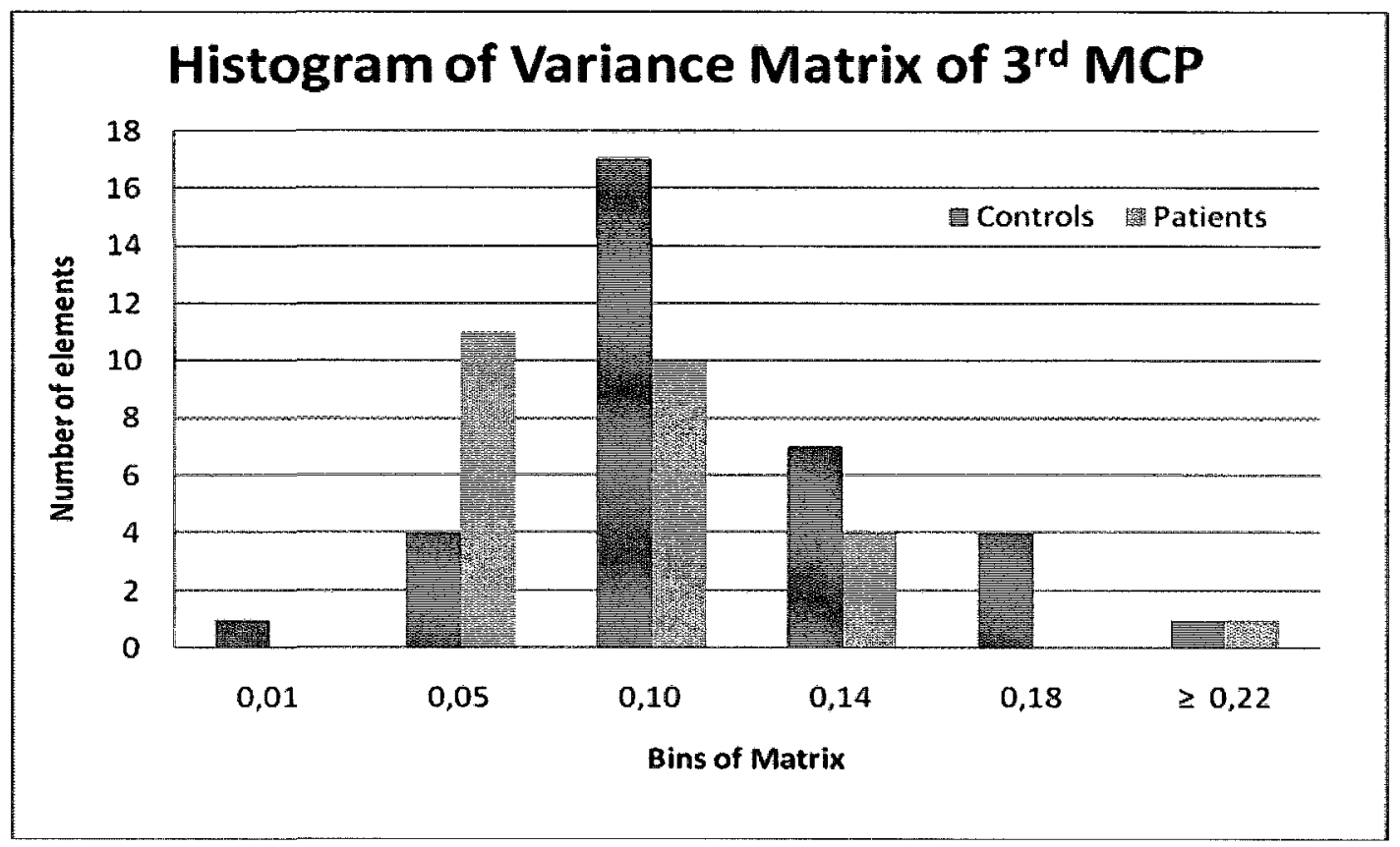

Figure 11-4: Histogram of the Variance Matrix for the 3rd MCP

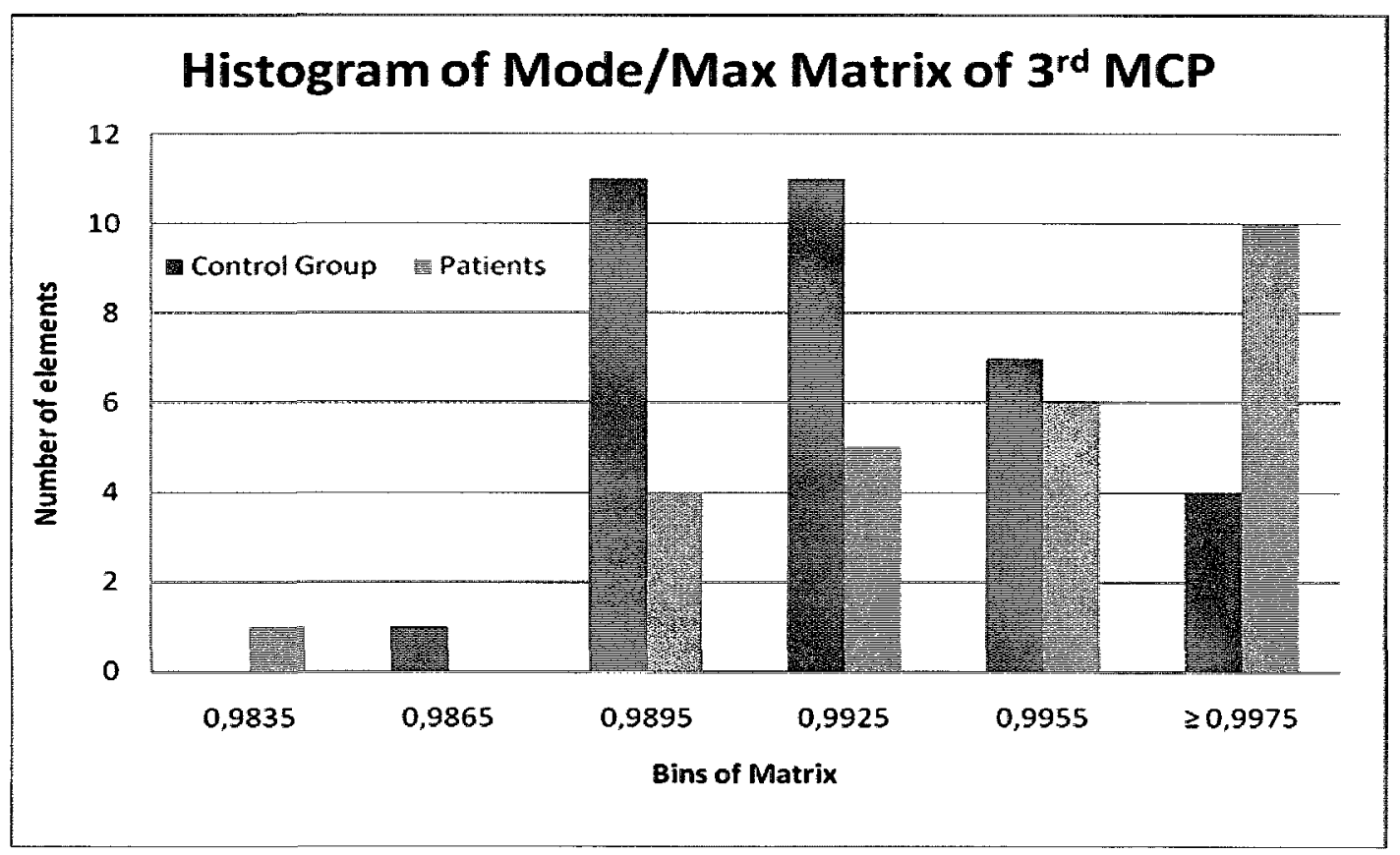

Figure 11-5: Histogram of the Mode/Max Matrix for the 3rd MCP 


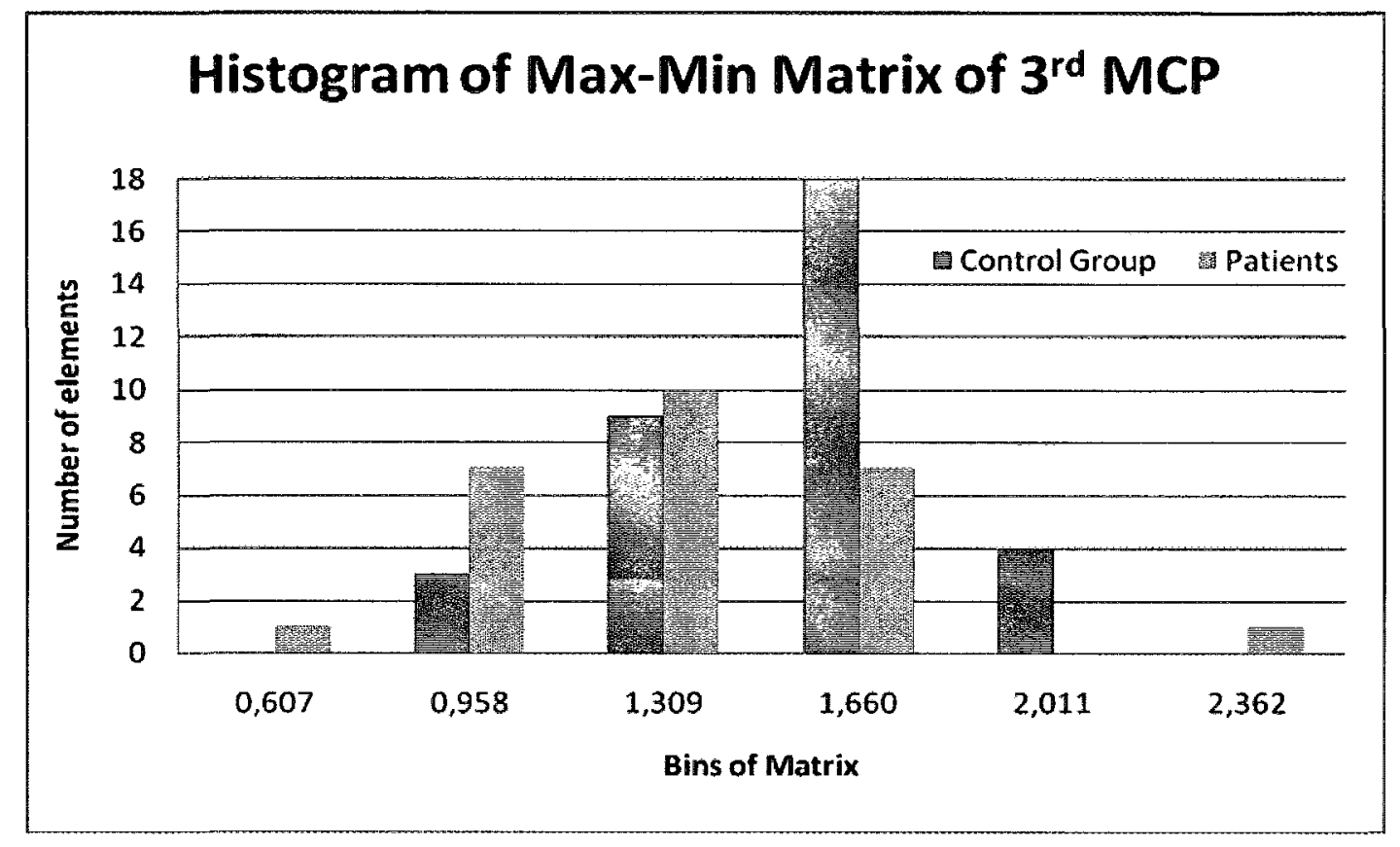

Figure 11-6: Histogram of the Max-Min Matrix for the 3rd MCP

11.3 Histogram of the Knee's Most Statistically Significant Datasets

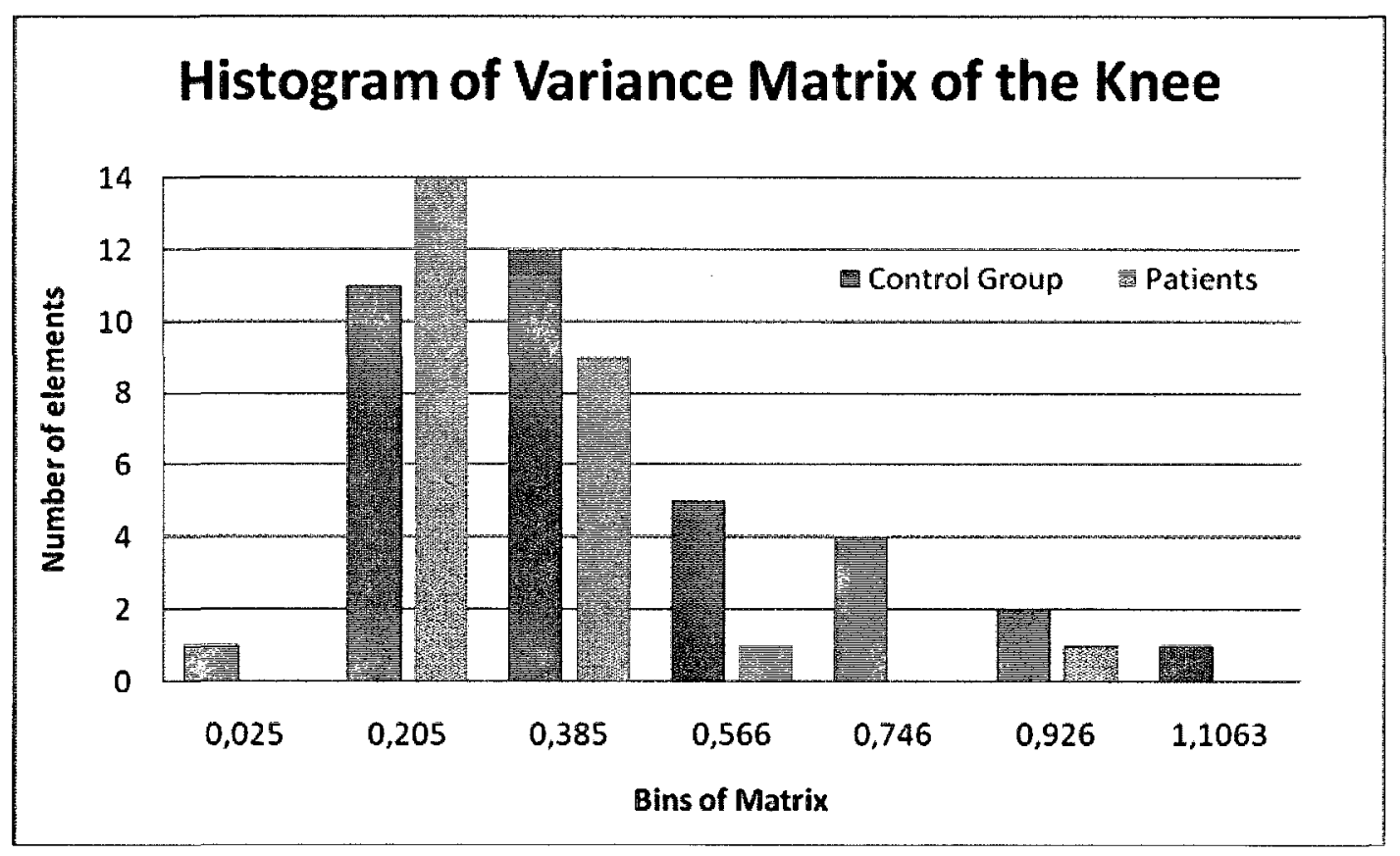

Figure 11-7: Histogram of the Variance Matrix of the Knee 


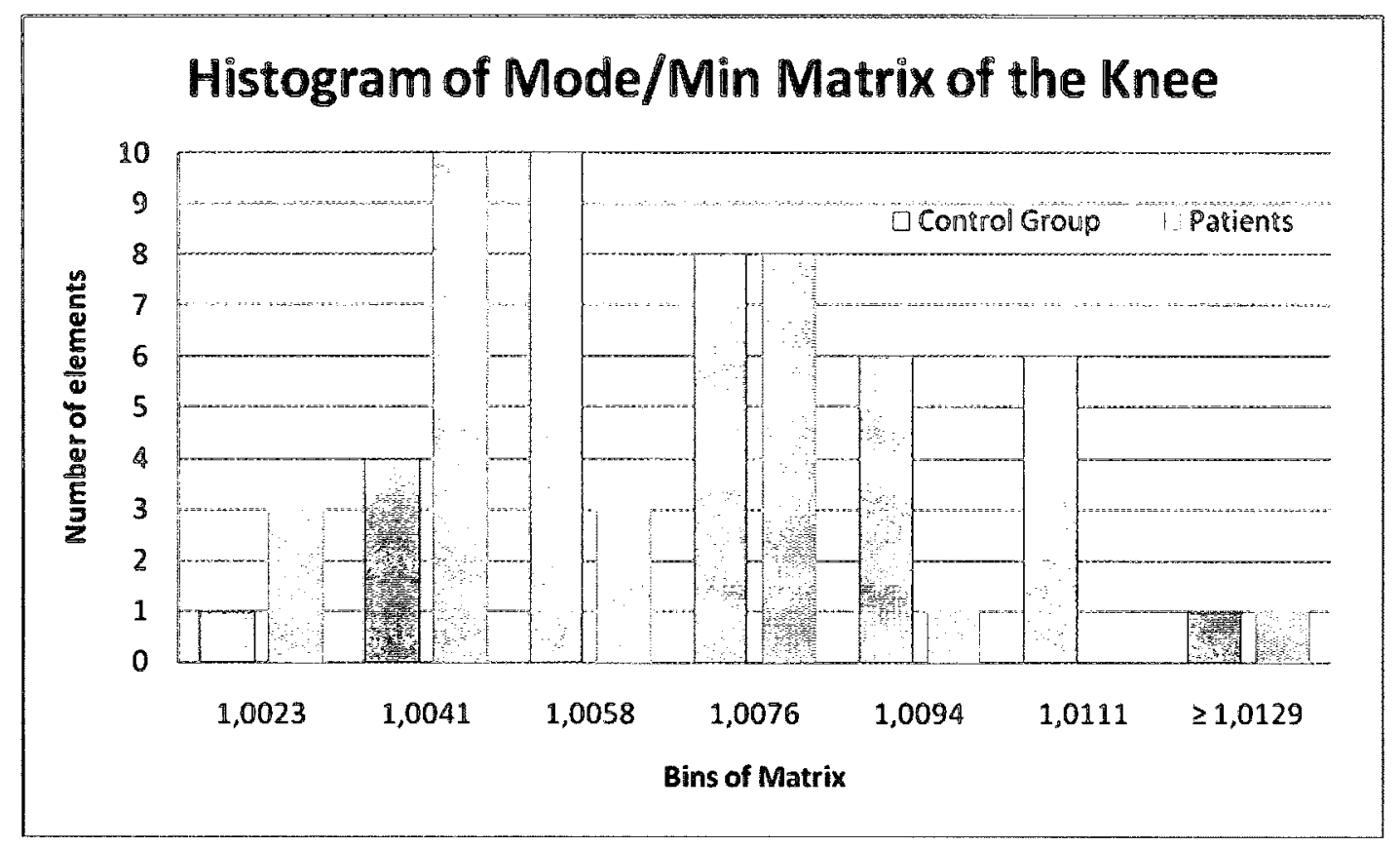

Figure 11-8: Histogram of the Mode/Min Matrix of the Knee

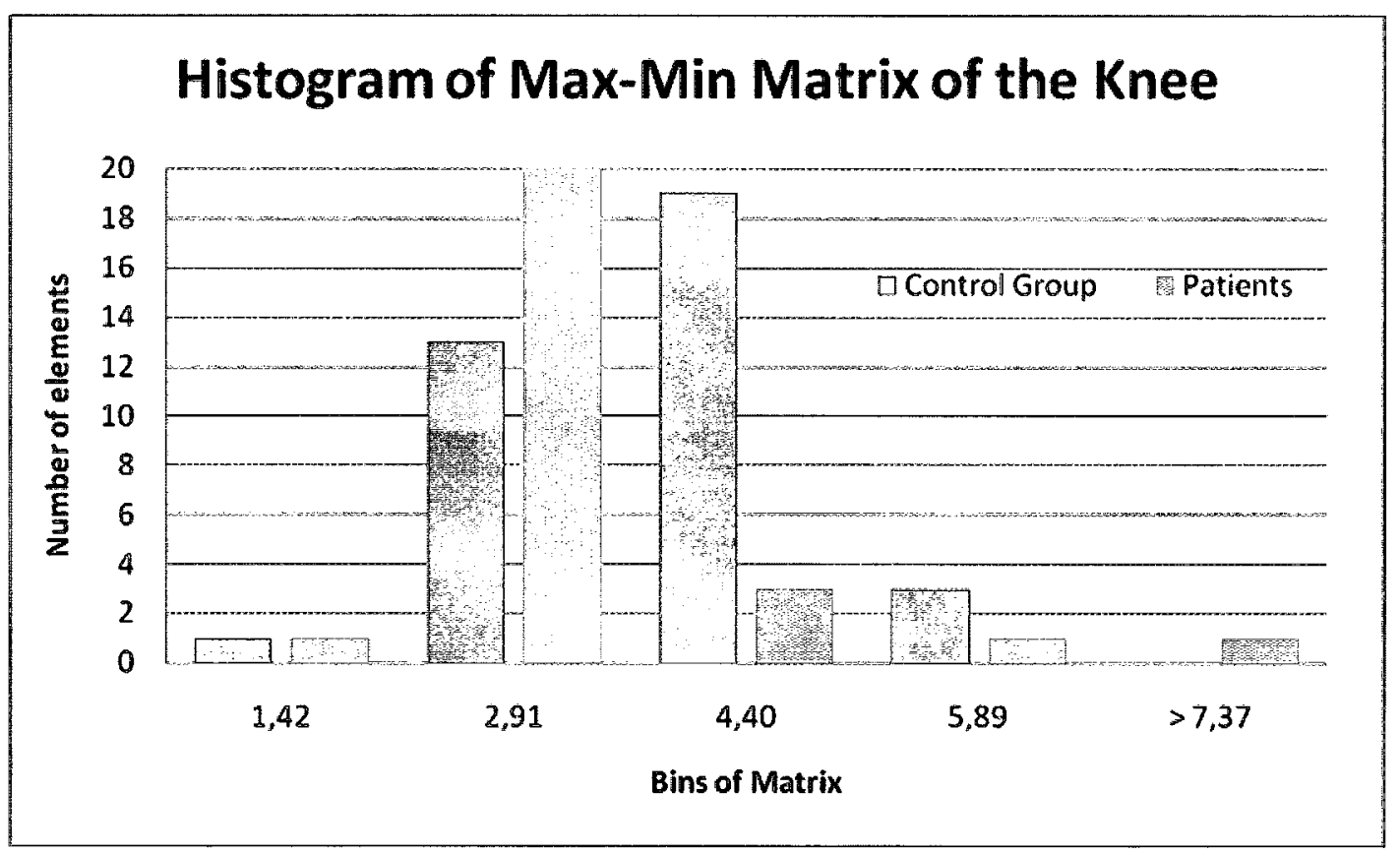

Figure 11-9: Histogram of the Max-Min Matrix of the Knee 


\section{Preliminary Results of Severity of Illness Measures of Rheumatoid Arthritis Using Infrared Imaging}

\author{
Monique Frize \\ Dept. Systems and Computer Engineering \\ Carleton University, Ottawa, Canada \\ School of Information Technology and \\ Engineering, U. of Ottawa, Ottawa, Canada \\ mfrize@connect.carleton.ca \\ Jacob Karsh, M.D. \\ The Ottawa Hospital-Riverside Campus, Ottawa, \\ Canada
}

\begin{abstract}
In the first phase of a large project we used an infrared thermographic camera to obtain accurate measurements of body temperature in joints of twelve human normal subjects (control group) and for thirteen patients diagnosed with rheumatoid arthritis (RA) by a rheumatologist. The ultimate goal is to create a low cost effective method to diagnose early synovitis. Temperature measurements of hands were analyzed with first order statistics. Results show significant temperature differences between control subjects and patients for every joint and hand portion measured. Future work will complete the analysis of knees, elbows, ankles, combine infrared imaging (IR) and intra-optical imaging (IO), and incorporate feature extraction and classification approaches to stratify patients into severity of illness prior to, and after receiving treatment.
\end{abstract}

Keywords- infrared imaging; thermography; measuring temperatures rheumatoid arthritis patients; comparing with normal subjects.

\section{INTRODUCTION}

Over 300,000 Canadians, 2.1 million Americans, and around 260,000 French are currently diagnosed with rheumatoid arthritis (RA), involving inflammation and symptoms like redness, swelling, heat, and pain. Within 10 years of diagnosis, $50 \%$ of patients have severe disability and life expectancy reduction from 3 to 18 years. With the aging of the population, the prevalence of chronic

\author{
Christophe Herry, Cynthia Adéa, Idris \\ Aleem \\ Dept. Systems and Computer Engineering \\ Carleton University, Ottawa, Canada \\ Pierre Payeur \\ School of Information Technology and \\ Engineering, U. of Ottawa, Ottawa, Canada
}

diseases and conditions is expected to increase even more over the next decades. Early diagnosis and treatment of RA could help postpone or prevent serious disability. Currently, diagnosis relies mainly on qualitative evaluation, medical history, examination, and laboratory tests. MRI (Magnetic Resonance Imaging) and HRUS (HighResolution Ultra-Sound) have been used with some patients to detect early synovitis. However these tests are not readily available to everyone and waiting lists in many countries are very long. Less expensive technologies providing quantitative assessment to diagnose and monitor therapy in joint debilitating diseases would be a major innovation in Canada and in the world. This explains the motivation to develop more accessible approaches for active prevention.

Visual marker-based monitoring systems still remain expensive and complex to operate. As a result, they are found mainly in particular cases in rehabilitation and for research. More tractable noninvasive approaches that simultaneously combine infrared imaging with movement extension measurement should be made available in large clinics and all hospitals to provide access to a major part of the population. Replacing expensive MRI or HRUS by the proposed technology would also have a major impact on the Health Care System; the current tests (MRI and HRUS) 
required for early diagnosis of RA could be replaced by the new imaging technique, thus releasing the expensive tests for other applications, likely leading to a reduction of waiting times for these tests. It is anticipated that the system will aid physicians in making earlier diagnoses and delay progression of the disease to advanced stages of deformity or disability, keeping Canadian and French citizens more active, at work and in their personal lives.

Inflammation and limitation of motion are two important parameters to monitor RA, along with pain assessment. The integration of heat distribution on the skin surface along with movement capabilities to be found in one diagnostic tool is innovative and seen as a reliable, quantitative and reproducible means of assessment of RA. This aspect is innovative and promising given that injury to bones, joints and muscles are known to recover slowly. Calibration of the automated measurement with disease level estimated by medical experts will also be possible providing that the correlation is sufficiently high with other clinical assessment tools. The resulting technology will support the translation of knowledge to physicians and other health personnel to help to improve or restore musculoskeletal health with quantitative measurements that can be classified, clustered and analyzed in order to observe tendencies and general trends to be compared with real clinical investigations. The ultimate goal is the integration of the multi-spectral approach into clinical application to become a diagnosis-aid that is affordable, efficient and easy to operate, and that can be widely used over existing health services networks.

From a clinical point of view, the technique of infrared photography may provide utility in several areas of care of patients with RA. a) Early diagnosis; currently, the diagnosis of RA is based on the clinical evaluation, specifically the demonstration of joint swelling by palpation. This method is at best crude and noted for substantial inter-observer and intra-observer variation. Refinement is provided by MRI and power Doppler. However, both of these methods are labor intensive, time consuming, expensive and also subject to the expertise of the individual performing the evaluation. Infrared photography may provide an alternate approach that could detect active inflammation at a time when the joints may simply be stiff and/or tender but not detectably swollen.

b) Prognosis; currently, the identification of biomarkers of disease severity and predictors of outcome is an area of active research. No single marker has been found to predict outcome well, perhaps with the exception of the persistent elevation of CRP despite therapy. The intensity of the infrared signal may be useful as measure of inflammatory load and a predictor of outcome.

c) Achievement of remission; the ability to suppress the intensity of the infrared signal may speak to adequacy of treatment. Currently, the goal of RA therapy is to induce the lowest possible level of disease activity. Measurements of disease activity rely on clinical parameters; however future goals are not simply to attain clinical remission, but radiographic remission defined as no progression of joint damage. Methods to demonstrate radiographic remission will rely on MRI, which noted above is an expensive approach. Infrared photography may provide an alternative to MRI in defining radiographic remission

Therefore, developing a less costly, non-invasive technology to provide a quantitative assessment of RA and to monitor therapy effectiveness in joint debilitating diseases has been the main motivation behind this work.

\section{LITERATURE REVIEW}

Over the past 50 years, infrared imaging has been applied to many fields. The major limitations encountered in early work were the lower resolution, large size of sensors, and the limited power of computers for the image analysis [1]. A few clinical studies demonstrate that heat distribution provides a quantitative measure of disease activity and especially of inflammation in knee joints of patients [2], [3], [4]. Correlation has been observed between heat distribution measurement and physicians' assessment based on degree of swelling, warmth, limitation of motion, grip strength, morning stiffness and pain score. Early studies mainly focused on knees and ankles to assess the effect of various treatments on RA patients. In this study, we analysed the temperatures of various joints in the hands, wrist, back of the hand, palm, thumb shaft, for both controls and patients. Our intent was to indentify how clearly we could separate patients from controls and to examine which joints are the most indicative of an RA condition in patients. 
Anomaly detection in infrared images is a challenging task. Often the key element of an automated anomaly detection system is the segmentation of relevant information from noise and background. Numerous algorithms are available in the literature for the segmentation of visible images, but these differ significantly from infrared images. The intensities of the latter depend on object temperature, object surface properties, surface orientation, wavelength, and are not necessarily uniform across an object, even if the temperature of that object is uniform. The environmental conditions at the time of imaging also influence the resulting images. Moreover, the range of intensities of infrared images is typically much less than for visible images, leading to low contrast, poor resolution, and less texture information. Key features that are successfully used in the processing of visible images cannot be used reliably when dealing with infrared images, thus the segmentation of the latter presents unique challenges and depends greatly on the application considered. Methods developed for automated tracking of military targets [5] or for predictive maintenance [6] do not perform well when translated to applications in medicine. Our group introduced a method based on morphological processing of edge maps using both strong and weak edges to recover the contour of faint parts of the object in low contrast and high noise situations [7, 8, 9]. Promising results were obtained for synthetic images of hands on three types of real backgrounds. The segmentation approach is part of a larger framework for automated identification of abnormal regions in infrared images of the human body and will be integrated into this project at a later stage. To date, our framework was applied to the assessment of pain [10], of musculoskeletal disorders in the arms and hands of computer users, and to capture the evolution of the temperature distribution on pianists' hands and arms during practice. We intend to incorporate this approach to the measurement of joints for diagnosing RA, once the first phase of the study described here is completed.

A second long-term goal is to develop a multispectral quantitative sensing approach to assess the severity of RA, combining infrared imaging (IR) and intra-optical imaging (IO). Building on previous work of our team to reconstruct the $3 \mathrm{D}$ position of human limbs from a segmented set of synchronized video captures without markers [11], precise gesture estimation can be achieved to evaluate the range of motion of a subject. It also provides a recording mechanism for temporal comparisons of the subject's capability of movement and dexterity. Our hypothesis is that this combination of infrared and passive intra-optical imaging will help in the early diagnosis of (RA) and to measure the effectiveness of therapy for patients treated for RA. The current paper addresses the first part of the work: measuring and analysing the temperature distribution in various joints of the hands and of other parts such as wrist, palm, and back of the hand. In subsequent work, the analysis will be completed on the other views taken of patients and normal subjects such as elbows, knees, ankles, and feet. Following this step, the superposition of the two imaging techniques will be developed.

\section{METHODOLOGY}

\section{A. Data collection}

Thirteen patients (nine females and four males) diagnosed with rheumatoid arthritis and eighteen control subjects were recruited after receiving the ethical clearance certificate from the hospital and the Universities. Six control subjects were eliminated from the total of eighteen volunteers for this preliminary analysis to maintain a database of subjects who were 50 years old or less; the six were between 60 and 70 years old. In the future, an in-depth analysis will be done with respect to the effect of age on mean temperatures of joints, but a quick observation was that persons over 50 had higher temperatures than younger subjects. All subjects (five females and seven males) and patients followed the protocol: not to use talcum powder, lotion, drug or deodorant on the skin on the day of the session; not to consume alcoholic beverages 12 hours prior to the session; not to consume hot beverages at least 1 hour prior to the session; not to use procedures such as acupuncture, transcutaneous electrical nerve stimulation (TENS), hot or cold patches, or any other form of physiotherapy at least 24 hours prior to the session. They were asked not to do intense physical exercises at least 4 hours prior to the tests, avoid prolonged sun exposure for at least one week, not smoke for at least 2 hours prior to the testing, and not wear any rings, necklaces, and bracelets during the session.

The room had a controlled temperature of around 20 degrees $\mathrm{C}$, with no windows. All test 
subjects wore light clothing (short-sleeved T-shirt and shorts); they removed their shoes and socks and were seated in the room without any of their limbs touching the other for a fifteen minute period before images were taken. This is to cool the parts of the body that were covered by clothing and have everyone in the same condition prior to the imaging session. The IR camera uses a solid state uncooled microbolometer focal plane array of $320 \times 420$ pixels, operating in the window $7.5 \mu \mathrm{m}-13 \mu \mathrm{m}$ of the far IR range of the electromagnetic spectrum. It has a $24 \mathrm{~mm}$ germanium lens with an anti-reflective coating. This gives a $24^{\circ} \times 18^{\circ}$ field of view with a minimum focus distance of $30 \mathrm{~cm}$. Its spatial resolution is $1.3 \mathrm{mrad}$. The thermal resolution is $0.05^{\circ} \mathrm{C}$ at $30^{\circ} \mathrm{C}$. The camera was connected to a laptop via a Firewire interface, thereby capturing sequences of 14bit digital thermal images at a set speed of 30 frames per seconds. Images were taken of the hands (dorsal and palm), arms, knees, feet (top and palm).

The preliminary analysis consisted of three steps:

(i) Calculating the mean temperature, the median, and the standard deviation for the twelve control subjects and thirteen patients for the following: Metacarpal Phalangeal (MCP) joints (all of the four joints together enclosed in a quadrilateral); Metacarpal Phalangeal joint of the third finger (MCP3); the third proximal interphalangeal joint (PIP3); the third distal interphalangeal joint (DIP3); the Mid Phalange (MP), the wrist, the back of the hand, and the palm; for the palms, the standard diameter was 120 pixels, which corresponded to approximately $4 \mathrm{~cm}$ in diameter. See Figure 1 for a drawing of the hand with the name of each joint. The mean temperatures for each view are presented in Table I with the first column reporting the results for the control group, the second column presenting the results for the patients, and the third column reporting the difference between the former two columns.

(ii) For the patient group, the joint temperatures are reported, as well as the mean temperature of all patients for each of the joints and parts of the hand. This is intended to see if a patient's joint temperatures are higher than, lower than, or equal to the mean temperature for each joint or part of all patients; it is our belief that patients whose joints are at higher temperatures than the mean temperature for all patients would have more serious inflammation and vice versa for those under the mean temperature. These results are presented in Table II. Of course, in the long run, it is the comparison of a patient's own temperature measures which will be important to determine if the disease if progressing or regressing at each visit.

(iii) The difference of the mean temperature between patients and participants for the right hand and the left hand were calculated for: $\mathrm{MCP}, \mathrm{MCP} 3$, the wrist, and the back of the hand. The results are presented in Table III.

Since there are between fifty and seventy-five images for each view, for each patient or subject, it was decided that for this preliminary analysis, the twentieth image for each view would be analyzed. The region of interest is indentified in the same way for each test and the mean and median is calculated for each. In the future, when the automation of the analysis has been completed, then it will be possible to average the data from all the images taken of each particular view.

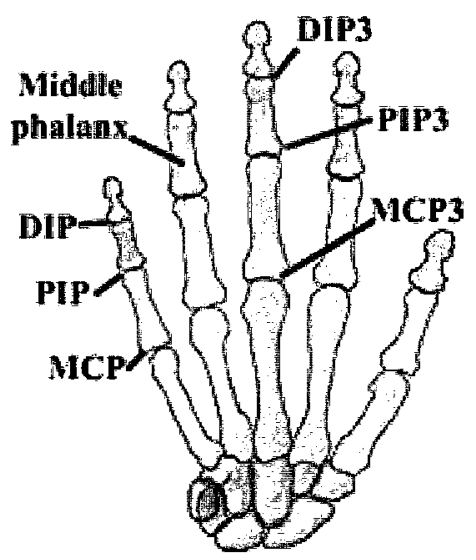

Figure 1: Joints of the left hand

During these imaging sessions, measurements were also taken of the following: Inside and outside of the left and right arms in a straight position, then inside and outside the right and left elbows in a bent position; the back as well as a front view of the bent right and left elbows. Similarly, the knee was imaged with the following positions: Back view of left knee and of the right knee, then a front view of these two joints; then inside and outside views of each knee. Finally, each foot was imaged with top view followed by a 
view of the sole. However the focus of this paper is mainly the hands; the analysis of all the other measurements will be part of future paper.

(iv) Three patients were retested in April 2009, nearly one year after the first imaging session done between April and June of 2008. Table IV shows the mean temperatures for MCP3, PIP3, and DIP3 taken at two different points in time (spring 2008 and spring 2009) for patient no. 1, 4, and 13.

\section{RESULTS AND DISCUSSION}

The results clearly show that the mean temperatures measured with infrared techniques were greater for all patients and all joints and hand parts than for the control subjects; see Table I. Greater temperatures were even seen at the DIPs, joints that are not often involved clinically in RA. On the day of the infrared testing, we did not examine each joint for clinical signs and symptoms of RA such as swelling or tenderness and are therefore unable to provide a correlation between clinical assessment and infrared signal. All infrared imaging tests were done at the University and in order to respect the requirements of the Ethics Review Board, these were to be separate at this time from the clinical evaluation. However, in the future, the new ethics proposal will suggest testing at the hospital facility and all patients will be assessed by a physician, by the imaging, and results compared to MRI tests (as ground truth). For this study, all patients had well established long-term RA and were being treated with disease modifying drugs. The physician's global assessment of disease activity at the time of the patient's visit was that the disease appeared to be less active in some patients (e.g. patients 1and 2) and very active in another (e.g. patient 7). However, by the time these patients participated in the imaging sessions, their RA status could have changed as indicated by the temperature measurements reported here. The preliminary analysis of the temperature measurements did not discriminate yet among patients with different degrees of clinical activity. Nevertheless, it is also known that clinical evaluation is often inadequate to evaluate joint inflammation except with approaches such as MRI testing. Other analysis approaches including classification techniques will be applied to this data in the near future to assess whether some discrimination of level of disease is possible.

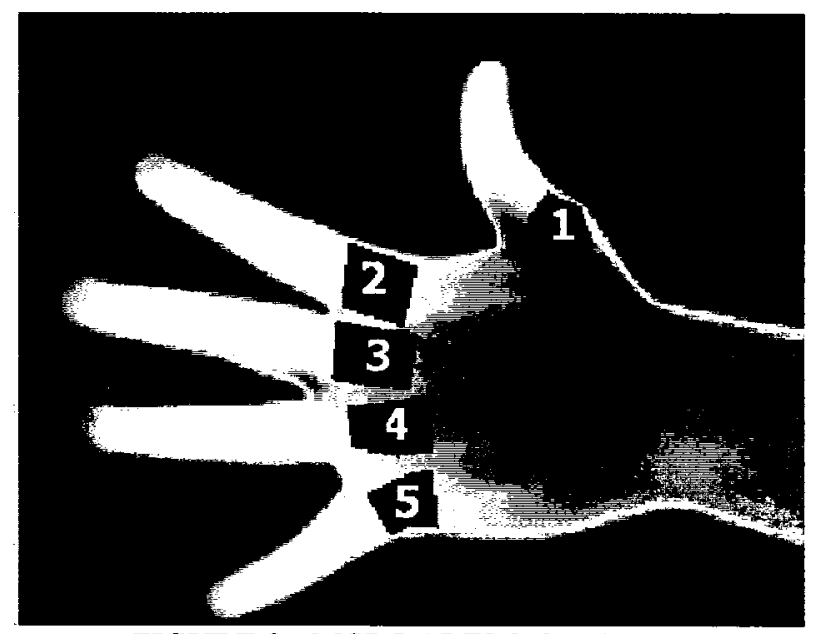

FIGURE 2: MCP LABELS ON A PARTICIPANT'S HAND

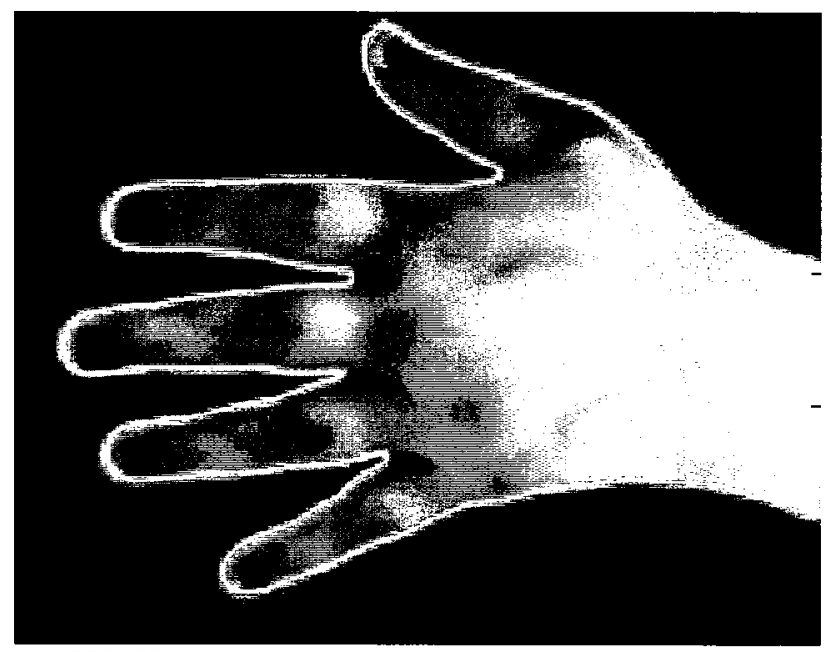

FIGURE 3: EXAMPLE OF A PATIENT'S HAND

TABLE I. MEAN TEMPERATURE FOR CONTROL SUBJECTS AND PATIENTS IN VARIOUS JOINTS IN LEFT HAND (DEG. CELSIUS)

\begin{tabular}{|c|c|c|c|}
\hline Part imaged & $\begin{array}{c}\text { Control } \\
\text { subjects }\end{array}$ & Patients & Difference \\
\hline MCP & 28.2 & 31.5 & 3.3 \\
\hline MCP3 & 28.1 & 31.4 & 3.3 \\
\hline Wrist & 29.7 & 31.9 & 2.2 \\
\hline Back hand & 29.5 & 31.6 & 2.1 \\
\hline PIP & 27.2 & 30.9 & 3.7 \\
\hline PIP3 & 27.5 & 31.0 & 3.5 \\
\hline DIP & 27.0 & 30.5 & 3.5 \\
\hline DIP3 & 27.3 & 30.6 & 3.3 \\
\hline MP & 27.3 & 31.0 & 3.7 \\
\hline Thumb & 27.7 & 31.7 & 4.0 \\
\hline PaIm & 30.5 & 32.5 & 2.0 \\
\hline
\end{tabular}


TABLE II TEMP. PATIENTS LEFT HAND (DEG. CELSIUS)

\begin{tabular}{|c|c|c|c|c|c|c|c|c|c|}
\hline $\begin{array}{c}\text { Patie } \\
\text { nt }\end{array}$ & $\begin{array}{c}\text { Test } \\
\text { date }\end{array}$ & MCP & $\begin{array}{c}\text { MCP } \\
\mathbf{3}\end{array}$ & PIP3 & DIP3 & Wrist & Back & MP & Palm \\
\hline $\mathbf{1}$ & $\begin{array}{c}\text { Apr. } \\
29\end{array}$ & 32.9 & 32.8 & 32.6 & 32.8 & 32.8 & 33.2 & 33.6 & 33.3 \\
\hline $\mathbf{2}$ & $\begin{array}{c}\text { Apr. } \\
25\end{array}$ & 32.9 & 32.9 & 32.9 & 32.7 & 32.5 & 32.8 & 33.4 & 34.3 \\
\hline 3 & $\begin{array}{c}\text { May } \\
\mathbf{8}\end{array}$ & 33.3 & 33.5 & 32.3 & 32.1 & 33.1 & 33.4 & 33.1 & 33.7 \\
\hline $\mathbf{4}$ & $\begin{array}{c}\text { Jun. } \\
\mathbf{1 2}\end{array}$ & 29.8 & 29.7 & 29.7 & 29.2 & 29.0 & 30.0 & 30.0 & 30.6 \\
\hline $\mathbf{5}$ & $\begin{array}{c}\text { Jun. } \\
12\end{array}$ & 28.8 & 29.0 & 27.2 & 25.8 & 29.9 & 29.8 & 28.0 & 30.0 \\
\hline $\mathbf{6}$ & $\begin{array}{c}\text { Jun. } \\
13\end{array}$ & 28.0 & 28.4 & 25.5 & 24.8 & 29.5 & 29.4 & 27.8 & 29.9 \\
\hline $\mathbf{7}$ & $\begin{array}{c}\text { Jun } \\
13\end{array}$ & 33.1 & 33.1 & 31.0 & 30.2 & 33.5 & 33.5 & 32.2 & 32.8 \\
\hline $\mathbf{8}$ & $\begin{array}{c}\text { Jun. } \\
16\end{array}$ & 32.1 & 32.2 & 32.6 & 32.1 & 29.2 & 31.1 & 32.6 & 31.8 \\
\hline $\mathbf{9}$ & $\begin{array}{c}\text { Jun } \\
17\end{array}$ & 30.7 & 30.1 & 31.6 & 31.9 & 30.0 & 30.8 & $3 \times 2$. & 31.9 \\
\hline 10 & $\begin{array}{c}\text { Jun. } \\
17\end{array}$ & 33.1 & 32.4 & 33.6 & 33.3 & 33.0 & 33.3 & 33.7 & 33.5 \\
\hline 11 & $\begin{array}{c}\text { Jun } \\
19\end{array}$ & 32.9 & 32.6 & 33.0 & 32.8 & 33.0 & 33.3 & 33.4 & 33.4 \\
\hline 12 & $\begin{array}{c}\text { Jun. } \\
21\end{array}$ & 32.1 & 31.9 & 32.3 & 32.2 & 32.2 & 32.5 & 32.8 & 32.9 \\
\hline 13 & $\begin{array}{c}\text { Jun. } \\
24\end{array}$ & 28.9 & 28.9 & 28.5 & 27.8 & 31.4 & 30.2 & 29.1 & 31.3 \\
\hline Mea \\
$\mathbf{n}$
\end{tabular}

TABLE III DIFFERENCE OF THE MEAN TEMPERATURE OF TWO HANDS FOR CONTROL SUBJECTS AND PATIENTS ( DEGREES Celsius)

\begin{tabular}{|c|c|c|c|c|c|c|}
\hline $\begin{array}{c}\text { Part } \\
\text { imaged }\end{array}$ & $\begin{array}{c}\text { Control } \\
\text { Left }\end{array}$ & $\begin{array}{c}\text { Control } \\
\text { Right }\end{array}$ & $\begin{array}{c}\text { Patient } \\
\text { Left }\end{array}$ & $\begin{array}{c}\text { Patient } \\
\text { Right }\end{array}$ & $\begin{array}{c}\text { Diff. } \\
\text { Contr } \\
\text { ol }\end{array}$ & $\begin{array}{c}\text { Diff. } \\
\text { Patients }\end{array}$ \\
\hline MCP & 28.2 & 28.2 & 31.5 & 31.6 & 0.0 & 0.1 \\
\hline MCP3 & 28.3 & 28.1 & 31.4 & 31.5 & 0.2 & 0.1 \\
\hline Wrist & 29.6 & 29.7 & 31.6 & 32.0 & 0.1 & 0.6 \\
\hline $\begin{array}{c}\text { Back } \\
\text { hand }\end{array}$ & 29.4 & 29.5 & 31.9 & 32.0 & 0.1 & 0.1 \\
\hline
\end{tabular}

TABLE IV. MEAN TEMPERATURE OF LEFT HAND OF THREE PATIENTS IMAGED AT TWO DIFFERENT TIMES (PATIENTS 1, 4 , AND 13): SPRING 2008 (A) and SPRING 2009 (B)

\begin{tabular}{|c|cc|cc|cc|}
\hline \multirow{2}{*}{ Part imaged } & \multicolumn{2}{|c|}{$\begin{array}{c}\text { A } \\
\text { patient 1 }\end{array}$} & \multicolumn{2}{|c|}{$\begin{array}{c}\text { A } \\
\text { patient 4 }\end{array}$} & \multicolumn{2}{|c|}{$\begin{array}{c}\text { A } \\
\text { patient 13 }\end{array}$} \\
\hline MCP3 & 32.8 & 33.3 & 29.7 & 27.5 & 28.9 & 32.4 \\
\hline PIP3 & 32.6 & 33.1 & 29.7 & 25.9 & 28.5 & 33.4 \\
\hline DIP3 & 32.8 & 33.5 & 29.2 & 26.8 & 27.8 & 33.3 \\
\hline
\end{tabular}

Again, although the physician could not be present at these second tests for patients 1,4 and 13 , it is evident from the tests results that patient 1 suffers from slightly more inflammation from the hand joint temperatures reported in Table IV. Patient 4 seems to be better and patient 13 seems worse.

\section{CONCLUSION AND FUTURE WORK}

The first phase of this research project enabled us to conclude that there are measurable differences in temperatures of hands in patients versus control subjects, establishing a proof of concept and leading to future work. Moreover it is interesting to note that subjects do not show much temperature difference between the right and the left hand joints or other hand parts (palm and back of hand), which is expected since they are not suspected of having any abnormality in either hand. For the next phase of the project, physician assessment, infrared imaging, visual imaging, and MRI studies will be collected and compared. Also, building upon C. Herry's work [7], future developments will include feature extraction processed through a pattern recognition system to optimize the classification of outcomes in terms of severity of RA and degree of changes at each visit. An index will be established, independent from the acquisition setup, to classify thermographic measurements into categories of RA diseases that correspond to currently used RA levels, as diagnosed by medical experts. A database will be created over this phase of research on patients already diagnosed with RA.

\section{ACKNOWLEDGMENT}

We thank the Natural Sciences and Engineering Research Council (Canada) for the research grant and the Faculty of Engineering and Design at Carleton University for the generous provision of two infrared cameras. The authors also wish to thank Ms. Seema Ambareen, graduate student at Carleton University (in our laboratory) for some of the most recent analysis of the data.

\section{REFERENCES}

[1] M. Aubry-Frize, G.R.C. Quartey, H. Evans, D. LaPalme, "The Thermographic Detection of Pain". Proc. of the 3rd Canadian Clinical Engineering Conference, pp. 82-83, Saskatoon, Sept. 1981.

[2] A.J. Collins, E.F.J. Ring, J.A. Cosh, P.A. Bacon, "Quantification of Thermography in Arthritis Using MultiIsothermal Analysis", Annals of the Rheumatic Diseases, Vol. 33, pp. 113-115, 1974.

[3] M. De Silva, V. Kyle, B. Hazelman, R. Salisbury, D.P. Page Thomas, P. Wraight, "Assessment of Inflammation in the Rheumatoid Knee Joint", Annals Rheumatic Diseases, Vol. 45, pp. 277-280, 1986.

[4] R.S. Salisbury, G. Parr, M. De Silva, B. Hazleman, D.P. Page-Thomas, "Heat Distribution over Normal and Abnormal Joints: Thermal Pattern and Quantification", Annals of Rheumatic Diseases, Vol. 42, pp. 494-499, 1983.

[5] B. Emisse, S.K. Rogers, M.P. DeSimio, and R. A. Raines, "Complete automatic target cuer/recognition system for tactical forward-looking infrared images," Optical Engineering, vol. 36, pp. 2593-2603, September 1997.

[6] S.-Y. Huang, C.-W. Mao, and K. Cheng, "A vq-based approach to thermal image analysis for printed circuit boards diagnosis," IEEE Trans. Instrum. Meas., vol. 54, pp. 3281-2388, Dec. 2005. 
[7] CL. Herry, R. A. Goubran, M. Frize, "Segmentation of infrared images using cued morphological processing of edge maps," in Proc. IEEE Instr. Meas. Tech. Conf., Warsaw, May 2007.

[8] CL Herry, M. Frize, RA Goubran. "Search for abnormal thermal patterns in clinical thermal infrared imaging," in Proceedings of the IEEE International Workshop on Medical Measurements and Applications MeMeA, Ottawa, Ontario, Canada, May 2008.

[9] CL Herry, RA Goubran, M Frize M. (2008) "Improving the Detection and Localization of Anatomical Landmark Points in Infrared Images Using Symmetry and Region Specific Constraints". Proceedings of the 25th IEEE International Instrumentation and Measurement Technology Conference (I2MTC08), Victoria, BC,May 2008.

[10] CL. Herry and M. Frize. "Quantitative assessment of painrelated thermal dysfunction through clinical digital infrared thermal imaging." Biomedical Engineering Online 2004, 3:19.

[11] S. Bériault, M. Côté, P. Payeur, "Volumetric Modeling with Multiple Cameras for Markerless Motion Capture in Complex Scenes". Proceedings of the IEEE International Instrumentation and Measurement Technology Conf. I2MTC08), pp. 359-364, Victoria, BC,May 2008. 